-

1.

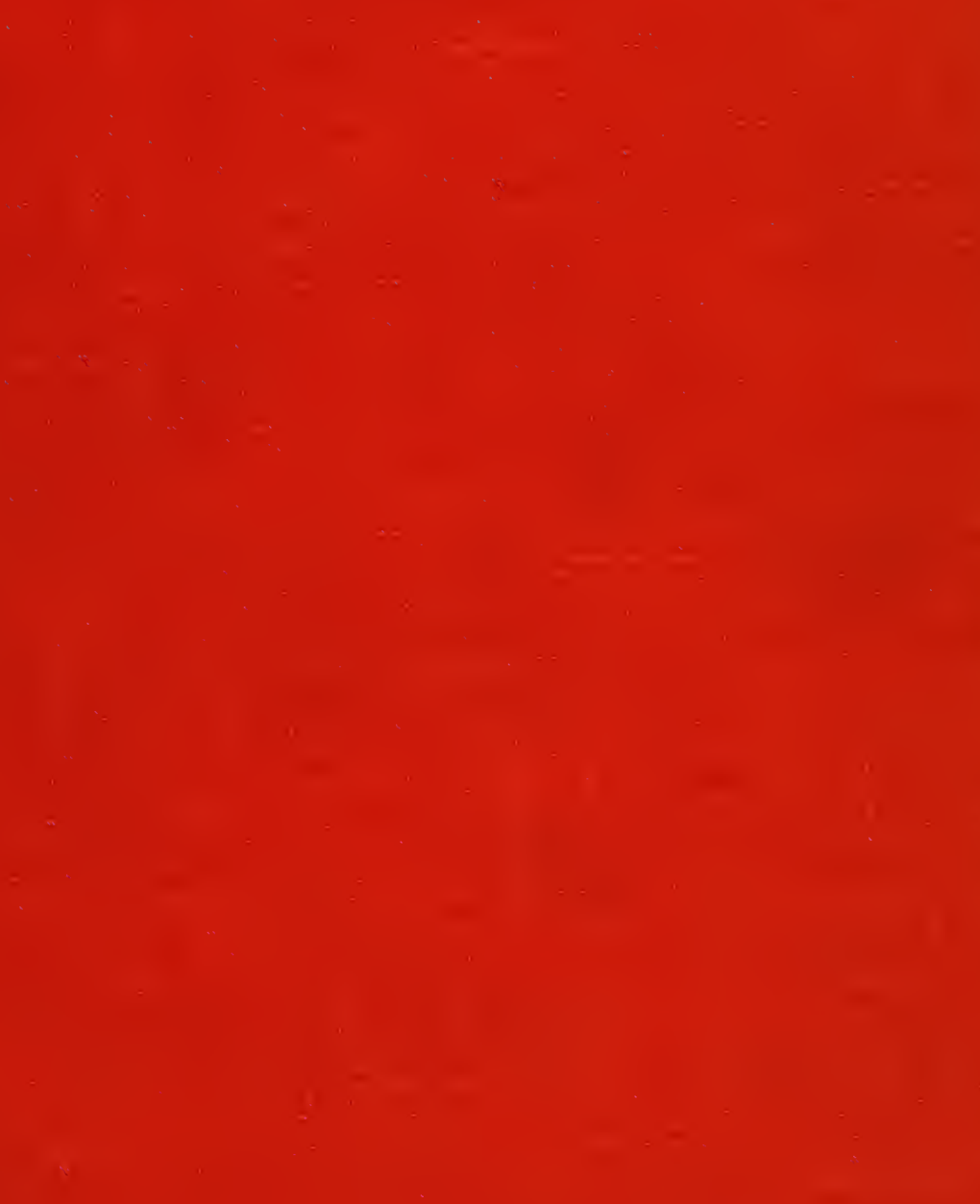




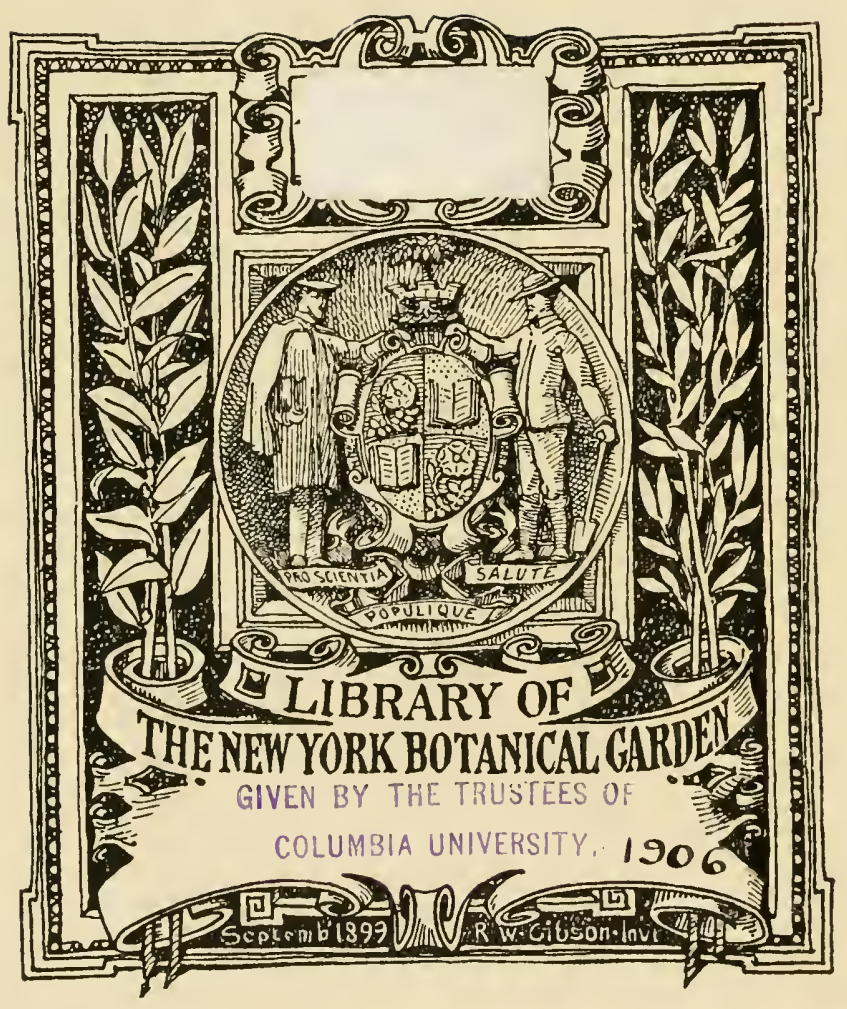








\section{$\mathrm{N}^{\circ} 1$.}

\section{GONTRIBUTION A L'ÉTUDE COMPARÉE}

\section{IDE LA FLORE}

DU

\section{MASSIF SCANDINAVE}

ET DU

\section{MagSIF GeHTRAL DE LA FBanGE}

\section{THÉSE}

Présentée el publiquement soutenue à la Facullé des sciences de Clermont-Ferrand

Le Juillet 1905

Pour oblenir te litre de Ducteur de l'Universile de Clermont-Ferrand

(MENTION SCIENCES)

PAR

C. S. CARLSON

JURY : $\left\{\begin{array}{l}\text { MII. POIRIER, Président. } \\ \text { GIROD } \\ \text { GLANGEAUD } \\ \cdots\end{array}\right.$

CLERMON'T FERRAND

IMPRIMERIE G. BONNET

1905 


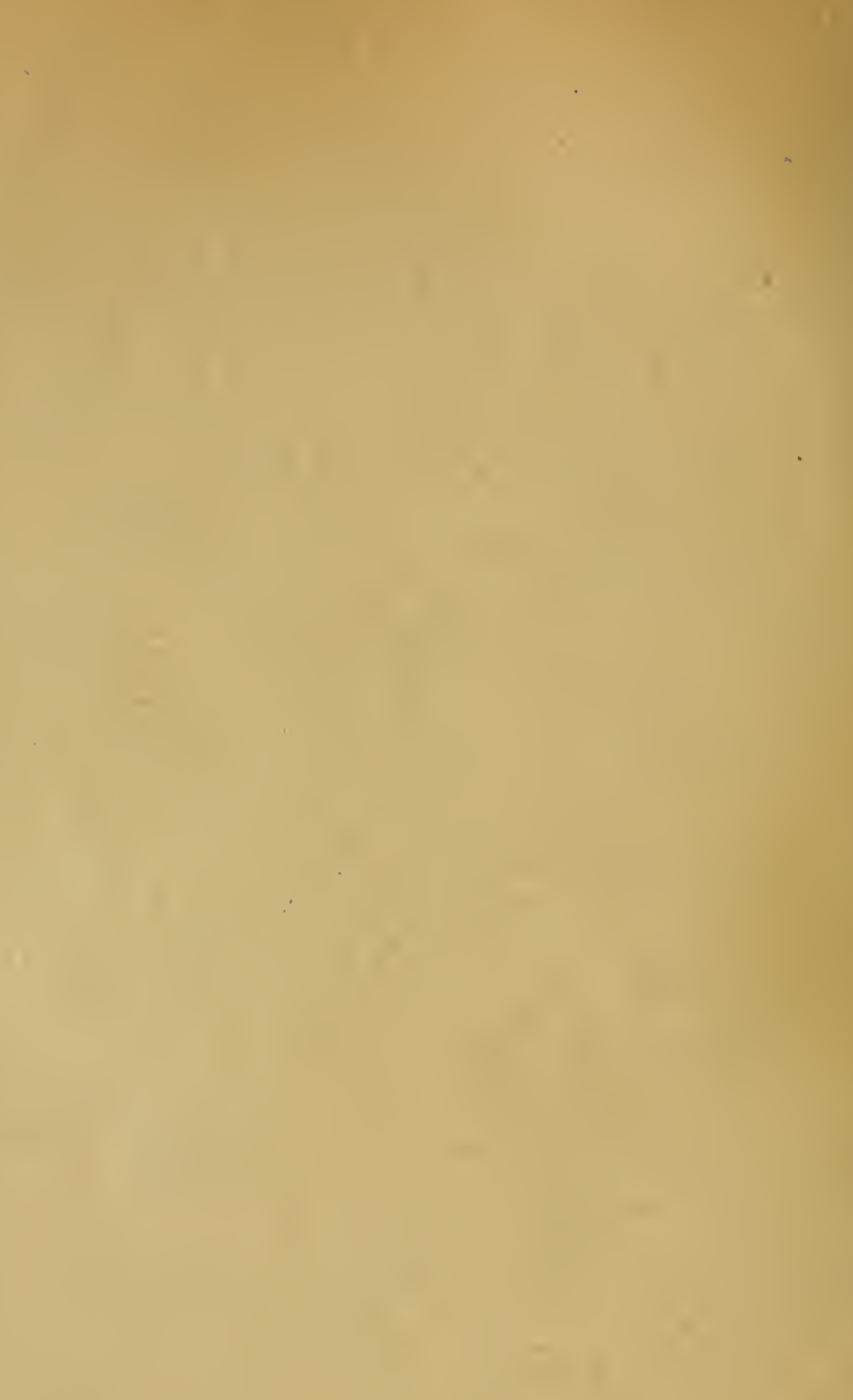




\section{CONTRIBUTION a L'ÉTUDE ROMPARÉE}

\section{DE LA FLORE}

\section{bu \\ MASSIF SCANDINAVE \\ ET DU \\ MASSIF GENTRAL DE LA FRANGE \\ THÉSE}

Présentée et publiquement soultenue à la Facultẻ des sciences de Clermont-Ferrand Le Juillet 1905

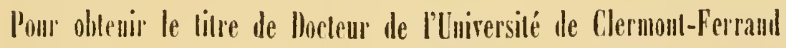

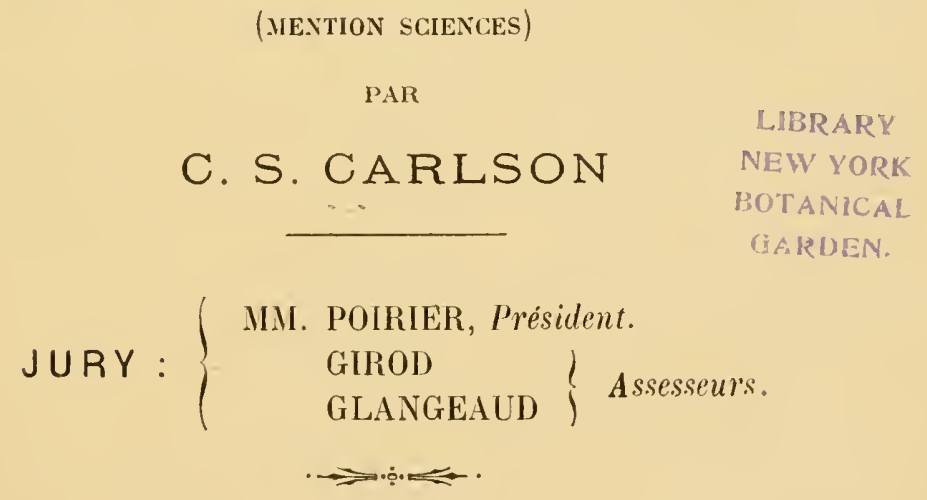

CLERIONT FERRAND

IMPRIMERIE G. BONNET 


\section{UNIVERSITÉ DE CLERMONT}

\section{FACULTÉ DES SCIENCES}
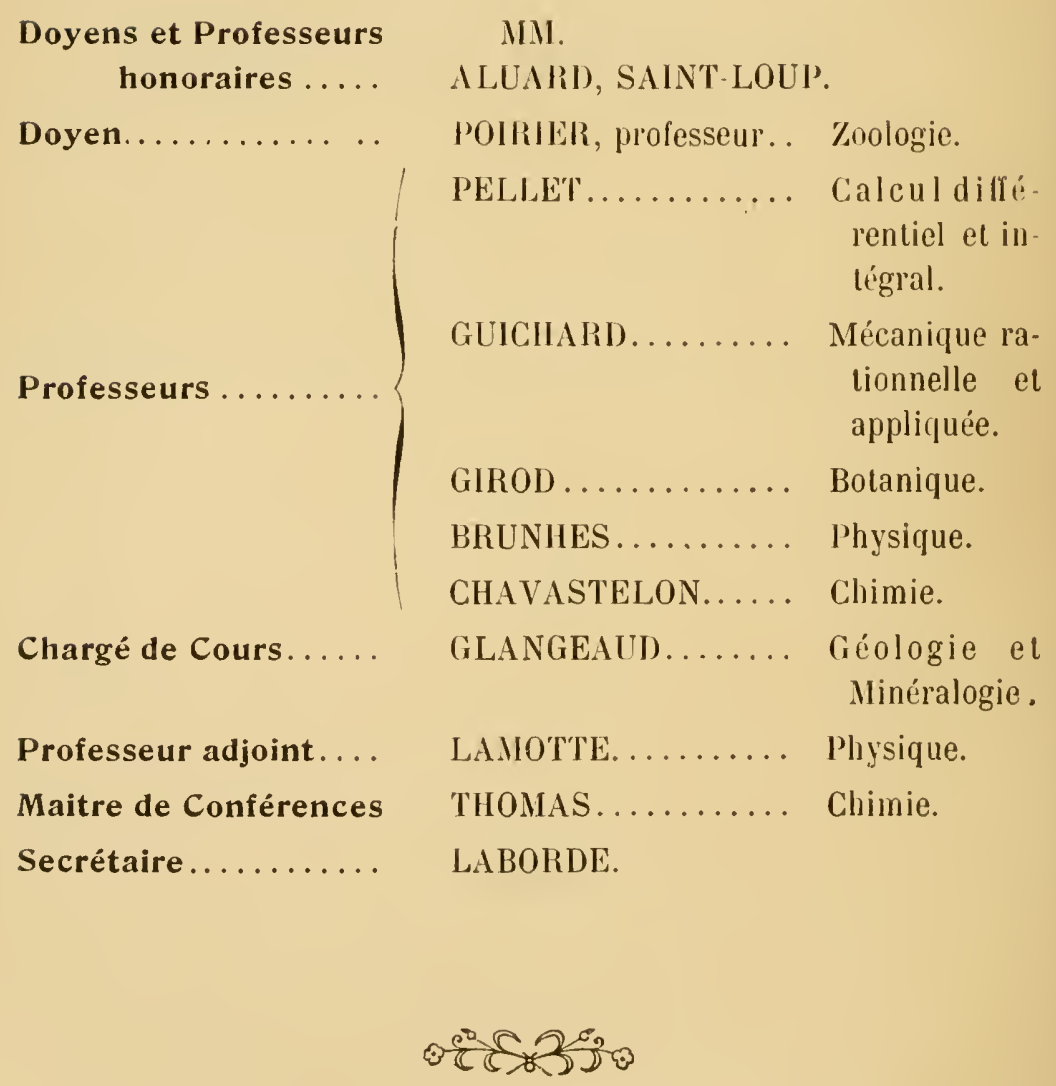


\section{A M. Le Dr Paul Girrod}

I'rofesseur de Botanique a la Facultí des Sciences Directeur de l.'École dF Médecine et de Pharmacie de Ciernont-Fiekmani

\section{et it M. le $D^{r} A \operatorname{xel} N$. Lundstrom}

Professeur de Botanigue a l.'Université d'Upsal.

Je dédie ce modeste travail, comme témoignage de respectueuse et profonde gratitude. 



\section{PREFACE}

Depuis cing années, je fais des séjour's en Auvergne. Mes promenades aux environs de Royat et dans la Limagne, mes excursions au Mont-Dore et dans le Cantal m'ont inspiré le désir de connaître plus profondément la géologie el la flore de ce beau pays. Avec mes connaissances de la Suède et celles acquises pendant un séjour en Suisse, j’ai nourri le dessein de prendre ì l'Université de Clermont les grades nécessaires en vue du doctorat is-sciences. Suivant pendant l'hiver successivement les cours des Universités de Zurich et de Berne, pendant l'été les cours de l'Universilé de Clermont, recerant d'Upsal des conseils el d'importantes collections, j'ai cherché à comparer mes impressions suédoises avec mes impressions auvergnates, à résumer ces impressions, et à compléter, par une étude microscopique des végétaux communs aux deux zones, ce travail d'ensemble fixant les modifications observées et en recherchant les causes.

Qu'il me soil permis de remercier is la fois le Professeur Docteur P. Girod, qui m'a ouvert d'une façon si généreuse ses laboratoires el a mis sa grande 
érudition et ses précieux conseils à l'appui de mes modestes recherches, et le Professeur Docteur Axel N. Lundström qui m'a documenté par ses remarquables envois.

Ma difficulté d'écrire le français d'une façon correcte m'a obligé à écrire en suédois et à demander l'aide d'un bienveillant traducteur; que mes juges me tiennent compte de cette difficulté de travail. Heureusement M. Lauby, attaché au laboratoire de botanique de l'Université a bien voulu m'aider dans le travail de révision de mes épreuves et je le prie d'agréer toute ma reconnaissance pour sa bienveillance et son aide précieuses. 


\title{
PREMIẼRE PARTIE
}

\section{LE MASSIF SGANDINAVE}

\author{
I. \\ GEOLOGIE - OROGRAPIIE \\ IIYDROGRAPHIE - CLIMATOLOGIE
}

La prestyu'ile Scandinare, appendice occidenlal de l'Europe seplentrionale, est comprise entre l'océan $\Lambda$ thantigue à l'onest, une partie de la mer Glaciale au nord, la mer Baltique ì l'est et se ratlache au continent par $u$ istlime lort large que marquent principalement les cour's de la Tana et de la Tornea.

La Suède est limilée an nord par la Nor'vège, il l'ouest par la Norrège, ledeltroit de Kattegat et par le Suml; an sud par la Ballique, à l'est par la Ballique el par le golfe de Bolnie et la Finlande. Elle est siluce entre le $55^{\circ} 21^{\prime \prime}$ et $69^{\circ}$ de latilude nord, sa longitule est comprise entre le 9" et le $22^{\epsilon}$ derré E. du meridien de Paris. I a Norrège est limitée an nord par l'océan gracial Aretique, a lonest par l'océan Atlantique, au sud farle détroit du Skagerak, à l'est une ligne comventionnelle la sépare de la Suède et de la Finlande.

\section{ESQUISSE GÉOLOGIQUE}

La Suède peut se séparer en deux rérrions, qui ont chacune leur facies différent: la régrion du nord qui demande de nouvelles explorations et la région du sud, qui est aujourd'hui bien mieux conmue.

En général ce sout les roches cristallines de la formation primitive, qui partout dominent et caractérisent les formations suédoises; elles sont recouvertes par les couches meu- 
bles de l'époque quaternaire: cependant dans la région du sud il existe quelques entassements de la série Mésozoïque.

C'est l'ètage "Silurien" qui partout offre la plus grande extension.

Dans les vastes régions du pays occupées par les roches de la formation primilive, les grneiss alternent avec d'autres dépôts sédimentaires de celle mẻme formalion.

Les éruptions contemporaines des granites et des porphyres y sont très abondantes.

Outre leur différenciation en gneiss rouge et gneiss gris, ces roches peuvent aussi être divisées en : Orthogneiss, Paragneiss et gneiss magnétique (en suédois: jeergneiss), ces derniers élant très riches en grains microscopiques de magnélite.

Une autre division de la formation primitive, probablement la plus récente, est fournie par une série de roches du groupe dit de l'eurite ou du häile flinta (petrosilex) passant de variétés à grains fins aux variètés les plus fines et les plus denses.

Ces roches sont importantes au point de rue industriel; elles renferment en ellet des masses considérables de minerais de fer qui se présentent en couches ou en plaques ellip soïdes; on y troure aussi des minerais de cuirre et de zinc.

Enfin dans leur roisinage on remarque sur plusieurs points des lits puissants de calcaire cristallin (marlure suédois); les rẻgions de gneiss en possèdent aussi.

Dans le groupe des roches éruptives il faut noler d'abord les porphyres el les greenstones (hyperites dialuas et trapp). Le plus grand territoire porphyrique de la Suede se trouve en Dalécarlie. On peut y reconnailre trois couches différentes: une inférieure ronge, rouge brune, très dure et riche en quartz, une moyenne de couleur sombre (Porphyre angitique), une supérieure se présenlant sous la forme de dykes.

Le greenstone apparait sporadiquement dans toutes les parties du pays, ordinairement sous la forme de massils ou de filons, parfois aussi comme couverture de conches sédimentaires; tel est le cas en Vestorgolhie. 
Vers la fin des temps porpliyriques il y a eu de fortes èruplions graniliques qui durèrent pendant une asse\% longue période.

Les granites affectent des facies variahles et paraissent appartenir ir des ìres diflérents quoique la masse principale date de l'spoque primitive.

Ils se présentent eu général par masses étendues, dont les principales, prises en granıl, peurent être considérées comme suivant la ligne médiane du pays du nord au sud; ainsi les granites sont prépondérants dans le centre de la Suède et le gneiss sur les côtes.

Les graniles peurent se diviser en trois grands groupes suivant l'époque de leur émission.

Les granulites micacées sont en général en contact géogrnostique avec les porphyres. La pegmatite apparait ça et là sous la forme de dykes ou de filons principalement dans le gneiss; parfois, comme dans le Skärgárd (Archipel) de Stockholm, elle est exploilée pour le feldspath pur qu'elle contient et qui est utilisé dans la fabrication de la faïence.

Les élages cambricn et silurien sont les seuls de la période paléozoïque qui soient représentés en Suèrle au point de vue fossilifere. Ce sont eux qui constituent le substratum des îles d'CEland et de Gotland; on les trouve aussi dans une foule de localités éparses des provinces de Scanie, Ostrogothie, Vestrogothie, Néricie, Dalécarlie Herjedal et Jemtland.

A la base des couches cambriennes se tronve des gres, tandis qu'à la partie supériemre ce sont des schistes. La zone de grès pent être divisée en grès à Eophỵton et grès à Fucoïdes. La première série, qui repose directement sur le gneiss est particulièrement riche en débris fossiles, tandis que le groupe à Fucoüles ne contient que quelques algues et des traces incerlaines de rers.

La partie inférieure des schistes cambriens se rapproche de la grauwache; sa composition est variable. Quant à la 
partie supėieure, de même que la tolalité des schistes à Olenus et à Dictyonema, ce sont presrue sans exception des schistes alunilères arec des couches peu considérables de calcaire bitumeux.

On peut distinguer 6 divisions dans les schistes a Paradoxides, chacune d'elle étant caractérisée par un type fossile : P. Kijeruifiy, P. Tessini, P. Davidii, P. oëlandicus, P. Forchhammeri, Agnosus locvigalus. Eilles contiennent des types nombreux des genres Ellipsocephalus, Arionellus, Allomacare, elc.

On y troure aussi des Brachiopodes.

Les ptẻropodes sont représentés par quelques espèces de Styolithus, tandis que les Gastéropodes, Ostracodes et Eponges y sont pen nombreux.

Les schistes à Olenus comprennent une foule de subdivisions, leur faune, oulre des Trilobites, renferme quelques Brachiopodes, Ostracodes et Graptolites.

Aux couches cambriennes fait suite le calcaire à Ceratopyge, qui renferme une faune franchement sous-silurienne représentée par les genres Megalaspis, Niobe, elc.

An-dessons se trourent des schistes et descalcaires à faunes diverses. Tandis que le schiste inférieur à Graptolites, le calcaire à Orthoceratiles, les schistes moyens à Graptolites, le calcaire a Chasmops of les schistes à Trinuclens sont soussiluriens, le schiste it Brachiopodes présente des faunes qui conduisent au silurien supérieur, les sehistes supérieurs à Graptolites assurent le passage définilil ì ce deruier terme.

Le silurien supérieur formé par des calcaires, des marues et dos grès est particulièrement bien représenté dans l'ile de Golland et dans les provinces de Scanie et de Jemtland.

Celte dernière localitè est pen riche en fossiles, à l'inverse des deux autres, ou les empreintes sont nombrenses.

Aussi durant la période silurienne les diflérentes formations de la Scandinavie du Sud étaient autres que celles de la région du Nord-Est. 
Nous avons ainsi demx lincies silmiens, l'un an Sull ol l'antre an Nord-Onest ; cederuer est anssi commu sous to nom de facies des contrés monlagnenses.

Comme type du premier on peut citer le silurien de Vestrogothie et surtout celui de Kinnekulle (colline suédoise).

Quant au silurien des hautes contrées montagnenses, son mancue de lossiles ne permet pas de le comparer aux autres horizons; cependant on y a trouvé des fragments de Crinoïdes.

Au Sud et dans la Suède Centrale le silurien est en couches horizontales sans crevasses ni fissures, ce qui indique que nul bonleversement n'a modifié celle région depuis les temps primitifs. Mais il n'en a pas élé ainsi dans les hautes contrées qui durant, et après la période silurienne, ont été le siège de dislocations puissantes, qui ont déchiré les conches et ont contribué is la formation du relief des monts scandinares.

Les formations mesozoïques ne se trourent que dams le. Sud de la Snède ef principalement en Scanie. Au Trias (Keuper) appartiement probablement certaines argiles non fossilitires de quelques localités scaniennes, sus-jacentes anx couches siluriemes et reconvertes par l'blage rhétien.

Les formations lublienues sont en partie la division la plus inferieure du Lias representes dans la meme province par les lits qui renferment les seuls gisements carhonilères de la Suède. Ces derniers sont en général accompagnés d'argiles réfractaires exploitées sur phusieurs points simulanément avec les charbous. Tel est le ras i I In̈ganïs, Billeshohm, Bjul', Stabbarp, Skromberga et Engelholın. Le nombre des couches houillères est variable et leur puissance respective oscille en géuéral entre 0,30 mètres et 0,90 mètres, souvent elle dépasse ce demier chiffre.

Les chrubous suétois sont it bien des égards supérieurs aux charhons anglais, car ils contiennent très peu de Soufre et de Pliosphore. 
Les autres couches de la formation se composent de grès ordinaires schisteux, d'argiles communes, reconverts partont de conches meubles (quaternaire) sauf au Nord d'Helsinglorg à Ramlüsa el le long de la vallée de Qvistofta. Les roches des denx premières de ces régions contiennent des couches peu puissantes de fer carbonaté lithoïde, dans lesquelles, de mème que dans le grès, on a principalement rencontré les quelques animaux fossiles trouvés jusqu'ici dans les formations susdites, tels que : Ostroea Hisingeri Nilss. Avicula Suecica Hébert, Mytilus Hoflmani Nilss., Mitylus psilonoti Quenst., Schizodus posteris Deffn. et Fraas., Cypricardia Nilssoni Héb..., et qui permettent de dater comme rhétien l'ìge de ces formations.

Ces dépôts marins, sans nul doute postérieurs aux couches carbonilères, sont accompagnés dans bien des endroits de végétaux fossiles l'emportant de beaucoup en richesse spécifique sur les formations équivalentes d'autres pays.

Par contre, les débris d'animaux sont très rares dans ces couches et se composent d'anodontes, de débris de poissons, de dents de sauriens et d'ailes de coléoptères Quoique plusieurs des végétaux en question se rencontrent dans le lias et quelques-uns même dans l'oolithe; l'immense majorité se compose toutefois d'espèces rhétiennes pures, que l'on a rencontrées aupararant dans les couches du même étage de la Franconie à IVilmsdorf en Silésie et à Seinstedt près de Brunswick. Elles montrent que l'àge de nos formations carbonilères proprement dite est rhétien.

Parmi les espèces les plus importantes déjà connues, on peut ciler les types Equisetum Munsteri, Schizonura hoërensis, Lepidopteris Ottonis, Dictyophyllum Munsteri, Acutilohum, obtusilobum et Nilssoni, Oleandridium tenuinerve, Maratliopsis Munsteri, Tœniopteris gigantea, Thinnfeldia saligna, Sagenopteris rhoifolia, Anomozamites minor, Pterophyllum oequale, Nilssonia polymorpha, Palissya Braunii, Baiera toniata... A ces types il faut ajouter une foule d'espèces particulières à la Suède. On rencontre également le Spi- 
rangium Munsteri, mais ordinairement dans les couches marines.

La tlore du lit honiller inférieur du T3juf, la plus riche en espèces, est contemporaine de la conche houillère la plus vieille d'Höganäs et tontes deux appartiement aux plus anciennes couches de l'étage carbonifère de la Scanie.

A la même période que celle des houilles scaniennes se rattache la formation isolée de grès située sur les rives du lac Ring, depuis longtemps connu des géologues sous le nom de grès de Hör. Composée principalement de grès et d'arkose, qu'on utilise comme pierres meulières, elle est très riche en végétaux fossiles parmi lesquelles il suffira de ciler comme les plus nombreux: Nilssonia brevis, Pterophyllum propinglum, Sagenopteris rhoifolia, Maratliopsis hocensis, Clathropteris platyphylla et meniscioüles, Dictyophyllum Nilssoni, etc. Les conches supérieures de l'étąrejurassique et les parties inférienres lu crélacé manquuent en Suède. En Scanie et dans les régions contigues du I Talland et du Blelinge on rencontre des calcaires et les grès appartenant anx groupes supérieurs de la craie, c’est-á-dire au Senonien et au Danien. Au Senonien appartiennent le calcaire d'Ignaberga avec Belemnitella subventricosa, Crania ignabergensis, Magas costatus et spatulatus; la eraie blanche de Fullstorp arec des éponges et des fynes lossiles rares el le grès vert (chloritique) de Köpingr, avec Ammonites Stoboi, Bellemnitella mucronata, Ananchyles ovatus, ele. Le calcaire Danien reuferme: Dromia rugrosa, Nautilus danicus, Cyprou bullaria, elc. et le calcaire de Saltholm arec Ananchyles sulcatus.

Les depots sédimentaires leptiaires n'existent pas en suède; cependant, sur le rivargesud de la Scanie se rencontrent des calcaires et des conclies sablomeuses.

Dans quelques localités existent des roches éruptives probablement d’ige tertiaire. C'est ainsi qu'au noul du Lac Ringr (Ringrajo), en Scanie, on tronve une quantité de petites cimes balsatiques éparses, mais l'andésite est peu abondante ; entin 
le rhyolite a été signalé an sud de la province de Smàland et sur le rivage sud-ouest du Mieusen.

Les formations quaternaires se rencontrent surtout dans la Snède moyenne et septentrionale. On peut les diriser en formations glaciaires et poslglaciaires.

Le dépot glariaire le plus ancirn est constitué par des graviers anguleux, reste des anciennes moraines appartenant aux glaciers continenlaux. Ces lépôls sont souvent remaniés et dans les rallées ils sont recourerts par des dépôts marins representés par des argiles ollaciaires d'une altitude moyenne de 150 mètres.

An-dessous de cette limite altitudinale, qui angmente dans la direction du nord pon' n'alteindre an sud de la Seanie qu'une épaisseur de 15 à 30 mèlres, se tronvent des parties dénudées de l'écorce rochense, des dépôts de sable el d'argile de la période quaternaire. C"est aux formations qualernaires qu apparliennent les "asar" ou Jongrs chapelels de collines de sable et de cailloux roulés qui se composent presque exchsivement de sable et de sédiments stratifiés mèlés de caillonx roulés et polis. Ces cullines courent dans la direction du Sud au Nord sur une longrueur de 200 a 300 lilomelres. Une partie de la ville de Stockholm s'élève sır un "as".

Les députs postglaciaires sunl constitués soit par des argiles manines qui ue se trouront qu'au roisinage des rotes ac:trelles, soit par des alluvions lluvialites el d'ean douce; de ces dernières formalions seuls les marais lombeux to es fréquents daus loutes tes rigions de la Suede offrent me réelle importance.

L'argile mlaciaire on l'argile fenilletée ne conlient more daus la suede de l'list dautres déhris fossiles que cenx de la Yoldia aretica, mollusque que l'on ne rencontre plus à l'état rivant que dans les parlies septentrionales de la mer glaciale. Dans la Suerle de l'Onesl l'arrile de Toldia est plus riche en débris oraniques. Elle y est en outre recourert sur quel- 
ques points de hancs coquilles contenant une foule de molluscfues des types Traphon clattiratus, Nattsia gronlandica, Follina proscima, Fecten islandicus, qui trahissent un climat arclique légèrement adouci.

La slation la plus importante est celle de Kapellbackarna (collines de la Cliapelle) près d'Uddevalla. Les dépôts des régions du Sud-Ouest et du Sud de la Scanie présentent une plus grande conformité arec ceux de l'Allemagne du Nord qu'avec les dépôts du mème âge dans le reste de la Suède.

Les plus anciens sont les lits stratifies de sable et d'argile, correspondant aux couches diluviales stralifiées de la partie sus-nommée du continent germanique.

Ils ne contiennent pas de fossiles el sont recouverts de moraines de fond plus argileuses que celles de la Suède moyemne par suite de lin nalure de la roche sous-jacente.

On o cru pouvoir les diviser en deux groupes: un groupe ancien et un groupe plus récent, dont la dissimilitude dépend d'une différence dans la direction du mourement de la glace. Entre ces lits de moraine ou dans leur intérieur mème, se tronvent, avec quelques mollusques d'ean douce, (Limnea, Pisidium, Cỵlıeridea) les plus anciens débris de végélaux purement aretiques, corresponlant à ceux que l'on rencontre an Spilzherg telles que Simule polaire (Salix polaris), la Divarle octopetale (Dryas octopetala), ele. A la fonte de la slace conti. nentale, les récions précitées de la Seanie ne prinent pas part an mouvenent d'ahaissement qui mit sous l'ean de la mer une wrande partie du reste de la Suede, lont les dépouts narins sont en conséquence remplacés en Scante par des dépûts d'eau douce. Les plus anciens de ces dépoits sont des lits stralifiés d'arifile el de suble, parfois de vase coquillère qui repose directement sur les dépots de moraines et contient soit des mollusques d'eau douce, soil tes plantes arcliques, telles que les types Salix polaris, reticulata et lierbacea, Dryas octopetala, Betula nana, des mousses, et parfois des cornes de reme. Au-dessus se présentent des dépóts de tourbes contenant ì leur partie inférieure des restes de pin commun 
(Pinus silvestris), d'Aurochs et de Bison, ainsi que des outils en Silex poli, le tout remplacé dans la partie moyenne par des débris du chêne à fleurs sessiles (Quercus sessiliflora) et des ontils en bronze, auxquels succèdent des outils en fer dans la partie supérieure. Le hètre acluellement très commun en Scanie manque dans les marais tourbenx et l'on peut suive ainsi l'adoucissement du climat de mème que l'immigration des plantes, des animaux et de l'homme depuis la période glaciaire jusqu’a nos jours.

A peu près contemporaine des tourbières de la Scanie est une formation de tuf calcaire à Benestad, contenant des feuilies des mêmes régétaux que ceux des tourbières, mais jas d'espèces arctiques. Il ressort des données géologiques, qui précèdent, que la possibilité de culture du pays dépend, pour une partie nolable de sa siluation en dedans ou en dehor's de la limite des dépôls quaternaires et marins et dans ce dernier, cas de la nature de la roche sous-jacente, celte dernière déterminant la composition du gravier anguleux qui forme le principal sol cultivable partout ou manquent les argiles marines. Si, du fait de sa nature pierreuse, ce gravier ne convient quaprès bien des peines et de grands labeurs a l'exploitation agricole, il n'en forme pas moins un sol qui n'est nullement ingrat, et partout où il se trouve fortement mêlé de terres argileuses et calcaires, il se prête admirablement ì l'agriculture comme, par exemple, en Scanie. Par suite de l'absence tolale de graviers et de cailloux, les argiles marines sonl, au contraire, d'un trarail facile el depuis longtemps déjà, elles ont élé utilisées en majemre partie par l'agrienllure. Dans les régions situees au sud des formations calcarilires, l'argile glaciaire presente tonjour's une tenenr plus on moins considérable (assez ordinairement de 20 à $30 \%$ de carbonate de claux qui en augmente notablement la fertilité comme, par exemple, chez les marnes de l'Upland.

Une différence sensible daus la répartition du sol arable 
se fait roir entre les régions où ce sol se compose de gravier de moraine et celles où il est formé de dépôts d'argile. Dans le premier cas les champs sont en majeure partie silués sur les pentes et forment rarement de grandes ètendues, mais à l'ordinaire de pelites surfaces i contours souvent irriguliers. Dans la région des argiles marines, au contraire, le sol arable est généralement concentré sur ces argiles, et ce qui reste est ordinairement abandonné à la végétation forestière.

\section{$2^{\circ}$ OROGRAPHIE}

Tandis que la Norrège est le pays des plateaux et des montagnes, la Suède est celui des longues déclivités et des plaines. Comme l'a dit le géographe Forssell, tout le massif scandinare ne peut être mieux comparé qu'à une vague prodigieuse qui se serait figée soudain au moment oì elle va déferler. D'après Erslew, un douzième seulement de la Suède se trouve à plus de 600 mètres d’altilude.

La charpente de la presqu'ile est formée par une vaste chaine de montagnes qui s'étend dı N.-F. au S.-O. en séparant les eanx de la mer de Norrège de celles de la mer Baltique; on la désigne généralement sous le nom d'Alpes scandinaves ou Dofrines; elle se compose de trois grands massifs :

$1^{\circ}$ Le Kjöjen qui comprend les $2 / 3$ le toute la chaine et s'ètend du Cap) Nord au mont Sylfjeld $\left(1,976^{\mathrm{m}}\right)$; son faite sépare assez exactement la Suède de la Norvège. Elle se compose essentiellement d'un énorme plateau de 800 à 1,000 mètres d'altitude, qui supporte des groupes montagneux isolés ou se rallachant au faite. Du còté de la mer Baltique, les gradins qu'elle forme se prolongent en longs plaleaux creusés de larges vallées parallèles bordées de contreforls qui s'effacent assez rapidement et dont quelques-mins seulement arrivent à la côte.

20 Du Sylfjeld, la chaine se recourbe de l'est à l'ouest jusque vers les sources opposées de la Drava el du Laagen ; elle prend alors le nom de Dofrines. Elle est plus élevée que la 
précédente; sa hanteur moyenne est de 1500 ì 1600 mètres, elle est constituée par des plateaux larges, marécagenx. Au sud elle s'étale en très larges vallées d'où descendent les cours d'eau les filus importants de la péninsule.

$3^{0}$ Des sources de la Drava, la chaine se dirige du N. au S. juscu'au cap Lindesnäs sous le nom de mont Lang ljeld.

C'est dans celte partie que se trouvent les plus vastes glaciers Snee-Bricen, Folge-Fond.

La limite des neiges éternelles dans les Alpes scandinaves est à 2.000 mèlres par $61^{\circ}$ à 1.170 mètres et à 700 mètres par $70^{\circ}$. Un $40^{\mathrm{me}}$ du territoire de la Norvège et $n{ }^{2.20} 0^{\text {me }}$ du territoire de la Suède sont occupés par la régrion des neiges perpétuelles.

lin ce qui concerne plus spécialement la Suède, on pent dire que le $62^{\circ}$ de latitude forme a peu près la séparation entre les deux pays, d'aspect diflérents. Ce sont au sud de vastes plaines ornées de grands lacs avec ça et lì le petits monts et de nombreux "asar". Au Nord, les pentes offrent un relief bien plus accentué bien qu'il y ait anssi de nombreux lacs.

D'une manière générale on peut dire que, de la crête des monts aux rivages de la Baltique, le sol s'aluaisse par ressauts de terrasse en terrasse; cest, a-1-on dit, un escalier aux marches très basses el tries larges. Du haut de la corniche suédo-norrégienne au golle de Bolnie, an nord de $62^{\circ}$, on descend ainsi du penchant oriental du Fjocll ou pays des montagnes jusqu'à la région des lacs sans nombre ef des marais; c'est ensuite la série des plateaux et l'on descend dans le Bolten, la plaine littorale large suirant les lieux, de S0 à 200 kilomètres; enfin c'est la mer. Le Botten est généralement à moins de 150 mètres d'allitude, les diver's plateaux ou les diverses Laponies sont, suivant les cas, à 300, 400 mètres on un peu plus; le Culmen de loute la Suède dans le massif du Kelına Gaize n a guère que 2.15j melres, le Sarekitjokio 2.140 mèlres, le Sulitelma alteint 1.900 mètres. 


\section{$3^{\circ}$ HYDROGRAPHIE}

Les côtes de la prespurile scandinare sont les plus découpées te toule l'burope, paticulieresnent dans la parlie norvégienne. C'est ici une inlinilé de golfes on de haies nommés ljorls, bordés de murailles a pic ; ils ressemblent i de véritibles fleures encaissés diuns des gorges étroiles el profondes, d'me largen de 7 is 8 kilomètres; ils s'enfoncent prolondément dans les terres et sont parfois agilés par des courants violents et irréguliers; le plus célẻre de ces cournuts est le Malstrom, au sud des îles de Loloten. Les fjords principaux sont cenx de Varanger, Porsanger, Salten, Trondhiem, Sogne, Hardangrer, Drammen, etc.

Les fjords sonl généralement libres de glaces en hiver; mème au cap Nord la température est moins rude que sur la còle suédoise, grite an Golfsträm, qui court le long de la cote, et de la barrière que les Dofrines opposent aux vents d’Asie. La côte suédoise ouverte aux vents qui ont traversés des plaines glacées à un climal lien flus rigoureux Plus basse que celle de la Norvège, elle est bordée d'une multitude d'îlots, d'écueils et de récifs; la parlie la plus méridionale on côle de Scanie n'en possiede pas, mais elle est très escarpée et le courant du Sud y est violent.

Les fjords sont parfois si bien protégés, que l'ean douce due à la fonte des neiges ou tombée en pluie se maintient à la surface en surmageant sur l'eau saline du lond.

Elle est si pure, que les algues du bord qu'elle baigne peu à peu ne tardent pas á périr et sont remplacées par des plantes d'eau rlouce à croissance hâlive. En hiver quand le continent ne verse phus d'eau douce dans le fjord, l'équilibre de salure se rélablit par l'écoulement de l'eau phus légère qui ra à l'océan. Les seuls qui conservent de l'eau douce à leur surface, sont cenx qui recoirent nne ou plusieurs rivières abondantes; ce sont en même temps des golfes marins et des lleuves. Certains fjords, interrompus par des barrières rocheuses, sont divisés en parties distinctes; c'est, en 
amont, un lac d'eau douce alimenté par les neiges et par les ruisseaux, tandis qu'en aval l'eau a conservée sa salure.

Depuis l'éporfue glaciaire, qui n'a pas encore cessé en Grönland, mais qui est terminée en Scandinavie, les glaciers ont reculé peu à peu dans l'intérieur des fjords, puis leur extrémité inférieure, que ne lavaient plus les flols, a remonté de plus en plus loin dans les dépressions ourertes sur le flanc des monts. A celte époque doit se placer l'immense travail du comblement des baies. Les eaux fluviales, apportant leurs alluvions, les déposaient en plages unies au pied des montagnes, tandis que la mer étalait en nappe de sable ou de vase tous les débris de rocher's qu'elle sapait de ses ragues. Celte transfcrmation du domaine des eaux en terre ferrne a fait des progrès considérables; c'est ainsi que, dans la convexité des côtes mèridionales de Norrège, entre le ljord du Porsgrund et la baie de Staranger, presque toutes les indentations du littoral ont disparu; il n'en reste que des criques, des pelits ports, des lacs, des étangs, des prairies humides. Lá, les glacier's ont cessé d'exister depuis beancoup plus longtemps que sur la côte occidentale tournée vers les vents plurieux de l'Atlantique. Des fjords oblitérés du sud de la Norvige aux fjords encore entiers des côtes septentrionales oì les glaces continuent de descendre jusque dans le voisinage de la mer, on peut observer tous les degrés possibles de transition. Ayant nevès, champs de glaces, forêts sans fin, mont de 1.800 ì 2.000 mètres, la Suède ne saurait manquer d'eaux comrantes, d'eaux dormantes; de fait, on ne saurait en compter les lacs, cliaque repli de terrain abrite au moins une nappe d'eau, clıaque vallée en contient une longue suite, on peut évaluer au treizième de la superficie totale de la péninsule la surface couverte des lacs dans la Scandinavie. Dans certaines régions de la Suède, notamment dans le Südermaland, entre Stockliolm et Norkköping, les lacs et les élangs se trouvent dans toutes les directions. Dans la Suède méridionale, la surface lacustre y occupe plus de la huitième partie du sol. Le plus vaste des 
lacs scandinaves est le Wener, qui comprend à lui seul un dixieme de toutes les eanx intériemes de cette région. Le second lac smédois est le Velter, qui a près de 2..000 kilomètres carrés. Quant au Mälar, qui a 1.688 kilomètres carrés, il se verse à Stockholm dans un ljord de la Baltipue, le Saltsjö. C'est un lac qui est toujour's golle par l'une deses extrémités; aussi, quand les vents d'Est souliennent le niveau des eanx lacustres, un courant maritime, l'Uppsjö, fait pénétrer un peu d'eau saline dans la partie orientale du Mülar. Arec ses nombreux détroits, ses treize cents îles, îlots on récils, cette mer intérieure doit être considérée, non comme une seule nappe d'ean, mais comme un ensemble de bassins séparés, ayant chacun son nivean propre.

La mer que gagnent ses "elfrar" ou flemves, frôle en zigzags les rivages par tant de fjords, elle est tellement lrangée de baies entre promontoires, que le littoral dn royaume n'a pas moins de 7.62i kilomètres de déreloppement dont 6.589 pour la Balticule et 1.035 pour les détroits. L'antre partie de la Scandinavie, la Norwège, décrit sur la mer 20.000 kilomètres de cotes. En avant de la rive suédoise, il y a d'antres grandes îles comme l'Áland, Golland, CEland et Borgholm. Les pelites iles rnu s'alignent en archipels d'illetles, de rochers, d'écueils, forment ce ruion approlle "Skärgàrd".

Outre les bassins lacustres de la Suede méridionale, on compte encore dans le reste de la Scandinavie 35 lacs occupant chacun plus de 100 kilomètres carrés; on pent citer le Torne-träsk, le Lule-träsk, le Stor-alvan, le Stor-sjö, le Siljan (l'œil bien de la Dalécarlie), le lac lälmar', le Mjösen, le lac Fämund (Norvège).

Lemraltilude vario de 1 is 5.6 melres. En hiver, its sont converts d'une ifrisse conche de glace qui se maintient pendant une moyenne de 100 à 200 jonrs, suivant la lititude de la contrée el la rigueur de l'hiver, mais il est tres rare que mème les étangs et les petits lacs sans profondenr gèlent jusłu'aux pierres du lit.

Les rivieres alimentées par les lacs innombrables de la 
régrion ne sont, pour la majeure partie, fu'mne suite de lacs de forme et de grandenr variables, qui, tantôt se rétrécissent emprisonness entre des parois, lantôt s'épandant dans la campagne en baies et en lacs latéranx. Leur débit est considérable comparalivement a l'étendue de leur bassin; il est dî aux conditions snivantes:

10 Pluies fortes sur tout le versant occidental et dans la regrion des sources.

20. Sol rocheux perméable a l'ean.

$3^{\circ}$ Evaporation faible dû ì l'humidité du climat.

Si l'on essaye d'établir une comparaison entre le régime fluviale de la Scandinavie et de la France, on remarque que la région du nord verse à la mer me quaulité d'eau bicn supérieure; néanmoins elle ne possède pas de conrs d'ran de li puissance du Rhône ou du Rhin; cela tient au relief de la contrée. Sur le versant norvégien, les cours d'eau à peine échappés aıx glacier's el anx nevès, sont recus par les fjords; sur le versant suédois les rivieres entrainées directement vers la Baltique par l'inclinaison du lerrain, ne peurent pas se rémnir en un seul faisceau. Celles qui débouchent dans le grolfe de Bohnie occupent des vatlées presque parallèles inclinées ver's le sud et dans la direction prise autrefois par les glaciers. Dans la Suède néridionale les eanx rayonment daus tons les sems vers les golles enviromants. Sanl le Gölaelf, aucun llewe ne rémnit a la lobis les eanix remues de la plaine à celles descendues de la montagne. Les riviores de la Srandinavie sont pour la plupart mieux réglées dans leur débit que les fleuves de l'Enrope continentale, grive aux lacs qu'elies traversent dans temr cours et qui en égalisent les crites.

l.e versanl oceidenlal ayanl me assez thande largenr clans la partie qui dome des eaux il bu mer glaciale, quelques rivières ont un cours de 200 kilomitres, mais elles ne traversent que les pays glacés desents du Fimmark. Citons le Pasvik, trop court pour elpe ravigable; puis la Tana, qui est narigahle jusqu'i: Seida el sépare la Norvige 
de la Russie; l'Alten traver'se une vallée asse\% peuplée, abondante en forêts et en mines; elle se termine dans un golle tris important. Entin, le Maals se jetle dans le Malangerljord. Puis la coite devient très étroite et les cours d'eau ne sont plus que des torrents coupés de chutes nombreuses impraticables i la narigation, traversant des vallées courtes, profondes, slériles, oi l'on troure à peine duelques habitants.

Nous nommerons seulement le Salten, qui linit dans le Saltenfjord, le Velsen, qui a 120 kilomètres de cours, le Namsen, la Nida, qui finit i 'Trondhiem, la Driva, qui s'arrète dans le golle de Christiansund.

I'lus au Sud, ce sont les fjords qui en faisant a l'infini des replis et des détour's sur leurs rives, sont les vraies rivières ou les moyens de communication du pays.

Le versant méridional est parcouru par de grandes rivières qui traversent un pays assez fertile et peuplé, mais elles sont mal encaissées, présentent de nombreuses chutes, el ne sont pas navigables.

On troure d'abord le Säterstal qui finit à Clıristiansund, le Numedal qui finit à Laurvig, le Drammen qui passe dans la vallée appelée I fallingdal, arrose Drammen et se grossit des eaux et des lacs de la Begna ; puis le Glommen, le plus considérable de la péninsule, ayant 500 kilomètres de longuenr. Descendu du mont Skarlind dans le Dovreljeld, il cont dunord au sud, en arrosant Rocraas et en traversant un pays samvage qu'on appelle le Hedemarken; la, il se grossit d'un grand nombre de lacs et d'alfluents; il y fait une série de chutes dont la plus considélable est celle de Sarpherg, ou il tombe d'une hauteur de 20 metres avec une largeur de 30 mètres à traver's des masses de rochers. Le Glommen après avoir passé à Kongsvinger est grossi sur sa droite par les eaux d'allluents et de lacs qui descendent du plateau oriental du Dovieljelds, ef court avec une effroyante rapidité, surtout après la fonte des neiges; il traverse ou forme encore plusieurs lacs el finit dans le Skagerrak près de 
Frederikstad. Le Tistedal sert de limite à la Norvège et à la Suède et finit à Frederikstad.

Le Gölaelf estle déversoir du lac Vener, qui reçoit un grand nombre de rivières dont la plus importante est le Klarelf. Le Götaelf sort du lac à Vennersborg; il se fraye un chemin au milieu de rochers qui forment des cataractes, dont la plus célèbre est celle de Trollhütlan; il passe à Kungrelf et se divise en deux branches, qui forment l'île d'Hissingen, et se termine à Golhembourg.

On trouve encore comme afluents du Kattegat, l'Etran, Nissan, qui finit à Halmstad et Lagan.

Le versant oriental, très large, est dans sa partie roisine de la mer presque entièrement plat, il est parcouru par un grand nombre de rivières qui vont parallèlement de l'Ouest à l'Est et présentent les mêmes caractères. Elles ont des eaux surabondantes et forment des lacs innombrables. On trouve d'abord une centaine de petits cours d'eau marécageux sans importance parmi lesquels on peut citer l'Helgeá, puis vient le Motala-Ström, une masse d'eau, qui est le déversoir de plusieurs lacs intérieurs, dont le plus important est le Vetter. Le Motala-Ström, en sortant du Velter, forme les lacs Boren, Roxen et Glan, et linit dans un golfe appelé Brảviken .

Le Dalelf est formé de deux grands cour's d'eau, le Vestel'dalelf et l'Gesterdalelf, qui se grossit de plusieur's lacs; il a 450 kilomètres de cours et se jette dans le golle de Botnie.

Le Ljusneelf descend de Sylfjeld, traverse un pays montueux el se termine après un cour's de 350 kilomètres.

Le Ljungran descend des mêmes montagnes, se dilate dans une multitude de lacs et de marécages et finit à Sundsvall. L'Indalself se forme dans les monts Kjölen par les eaux de plus de 40 lacs, traversés par autant d'alluents; il se grossit encore dans sa partie moyenne de son cours par le Storsjö (grand lac); il devient alors un fleuve large et rapide, qui coule ensuite dans un lit assez régulier pour finir dans lẹ golfe de Botnie. 
L'Ȧngermanelf a les mômes caractères que l'Iudalself; il est formé ou grossi par une infinité de lacs et d'atlluents, n'arrose aucu lien considérable, a, vers la fin de son cour's une largeur remarquable el linit près de Iternösand. Ce sont ensuite les solitudes du Lappmark, parcourues par les lapons.

L'Umeelf est formé comme les précédentes par la réunion de nombreuses eaux stagnantes, mais dans la parlie moyenne de son cour's elle a un lit assez régunlier; elle se grossit sur sa rive gauche du Vindelelf, qui a un cours aussi long.

Elle se lermine i Umeá après un cours do 300 kilomètres.

Le Skellefleelf est le déversoir du lac Stor-Afran.

Le Piteell est aussi le déversoir de plusieurs lacs; elle a un com's assez régulier, mais dans un pays presque désert, et finil à Pilea. Le Luleell sort l'une série de lacs, qui ont ensemble plus de 100 kilometres de longueur; il finit à Lulè่.

Le Kalixell a un cours plus régnlier que celui des rivières précédentes; il se grossit de peu de lacs, traverse un pays presque entièrement glacé ou désert et linit à Nynäs.

Le Torneelf se grossit du Muonio et forme avec celte rivière el la partie supérieure de son cours la limite entre la Suede et la Finlande. Elle se termine au fond du golle de Botnie, à Torneà.

\section{$3^{\circ}$ CLIMATOLOGIE}

La péninsule scandinave forme avec le Grönland la porte marine, qui fait communiquer l'océan Allantique et l'océan Glacial.

Dans la péninsule il y a de hautes forêts, des cultures de pommiers, de poiriers, de prunier's et de cerisiers. Cepen(lant, une partie de la Scandinavie (environ 155.000 kilomètres carrés) est comprise dans la zone polaire et pendant l'hiver la nuit s'ajoute à la nuit en ténèbres continues. Mais aussi l'Europe méridionale ne connait pas cette douce clarté 
d'une matinée ou d'une soirée du Nord, cette lente disparition des rayons du soleil reflétés par l'océan et ces nuits encore embellies par la faible lueur du crépuscule, an moment où l'aube matinale se montre à l'Orient. A Stockholm, les plus longs jour's et les plus longues nuits sont de 18 heures et demie et les plus courts de 5 hieures 5 i minutes; pour Lund nous avons 17 heures 18 minules et 6 heures 56 minutes, pour Tornea 21 heures el demie el 2 heures ef demie; enfin, près d'Olser-Torneá, on peut voir le soleil pendant toute la nuit de la Saint-Jean.

Les diver'ses parties de la Norvègre éprouvent une grande différence dans la longueur du jour, suivant qu'elles sont plus ou moins rapprochées de l'océan Glacial.

Dans la partie méridionale, le plus long jour est de 18 heures et demie et le plus court de 5 heures el demie; au centre ils sont de 21 heures et de 3 heures, mais dans la partie la plus septentrionale le soleil reste en été sur l'horizon pendant plusieurs semaines et en liver il est invisible pendant un égal espace de temps. Les plus longues nuits sont, pendant l'été, de 2 à 3 heures, et les plus longrs jour's, pendant l'hiver, sont de la même durée. Les nuils d'hiver de la Norvège septentrionale sont éclairées ou par la lune ou par l'éclat resplendissant des auror'es boréales.

Les vents chauds du sud-ouest et du sud repoussent vers le nord les lignes isolhermiques de la Scandinavie et leur font suivre la côte presque en sens inverse de leur direction normale. Les vents dominants pendant l'hiver, voire mème pendant le printemps et l'automne. sont des brises qui, des vallées et des ljords, se dirigent vers la surface de la mer dont la tempèralure est toujour's supérieure au point de glace. En été, au contraire, les vents se portent de l'océan ver's les régions échıuffées de l'intérieur.

Les orages qui ont lieu rendant la saison chaude sont peu nombreux et rarement accompagnés de grêle.

Les lignes isolhermiques tracées grâce aux stalions météorologiques de la Suede et de la Norvège, présentent en été 
comme en hiver la même forme générale, elles se développent presque parallèlement à la côte occidentale puis décrivent leur principale courbe dans le sens du sud-ouest, comme le littoral lıi-mème.

Loin des rivages, loute la péninsule se trouve comprise dans les limiles de l'isochimène de zéro. Calculée pour l'année entière, la ligne de congélation embrasse l'intérieur du Finmark nor'végien el du Norrbotten suédois, plus au sud le platean du Dorre et quelques autres massils autour de Rörcis.

$\Lambda$ lors que la température moyenne anmelle de tonte la Norvège est de 2 J centigrades, celle de la Suede est d'enviroll $6^{\circ}$.

Les écarts journaliers, mensuels et annuels de température sont considerables; de $10^{\circ}$ sur la côte entre juin et juillet, ils sont de $30^{\circ}$ dans la Laponie suédoise; du cap Stat au Lofoten, la varialion de la température entre les diver's mois de l'année est de 12 à $13^{\circ}$; à Haparanda, la varialion est de 320 Dans ces régions du nord, les élés sont à peu près aussi chauds que dans la Suede méridionale, mais les hivers y sont beaucoup plus froids et on y a même subi des températures moindres de $50^{\circ}$. Les glaces épaisses qui se forment pendant l'hiver dans les marais tourheux de la Laponie se maintiennent parfois durant loute l'année.

La quantilé des pluies est variable suivant les versanls considérés. Dans les îles de Lofoten, il pleut en moyenne un jour sur deux; à Bergen et sur le littoral, au nord dı cap Stal, la chute annuelle est de deux mètres; au-deli des glacier's et des nevés, la moyenne d'eau est de un mètre; à Tromsü, à Christiania, les pluies n'alteignent qu'un demimètre.

Dans toule l'étendue de la Suède protégúe à l'onest par le faite des plateaux el des monts Scandinaves, la moyenne d'eau est de 500 millimèlres.

La limite des neiges persistantes descend plus loas sur les côtes occidentales des monts de Folgefonden et de Tristedal 
attiédies par les vents marins que sur les déclivités tournées vers le froid orient; cela tient à l'humidité fournie par les courants aériens de l'ouest et du sud-ouest; mais du sud au nord, la limite des neiges s'abaisse régulièrement de 1.250 ou de 1.500 mètres sur l'un ou l'autre versant de Folgefonden à 140 mètres et même à 750 sur les montagnes voisines du cap Nord.

Les eaux océaniques réchauffées par le Golf-Ström viennent frapper la zone extérieure de la péninsule scandinave; ce courant dirigé du sud-ouest au nord-est donne à la Norvège un climat propre. La température de l'eau dans les fjords est plus élevée que celle de l'atmosphère ambiante; la différence atteint jusqu'à $13^{\circ}$ en janvier ; cela lient à la disposition des bassins qui ne reçoivent de l'océan que des eaux tièdes; ainsi, tout le littoral de la Norvège est formé par une ceinture d'eau chaude dont la température varie suivant les saisons ; elle assure à la région des fjords et des îles un climat relativement doux, mais avec des lorumes et des pluies fréquentes. Le froid ne se fait sentir dans cette région que lorsque règnent les vents d'est qui traversent les hautes montagnes ou celui du nord qui vient de l'océan glacial; mais les exhalations salines de la mer nuisent à la végétation. Toutefois, en raison de sa latitude et de son altitude générales, le climat de la Norvège est essentiellement froid, car, comme nous l'arons dit, l'isotherme de $0^{\circ}$ englobe tous les plateaux de l'intérieur où la température hivernale atteint de $10^{\circ}$ à $30^{\circ} \mathrm{C}$.

Quant au climat de la Suède, s'il est généralement très froid, il présente des différences très grandes au midi et au nord; c'est ainsi que la glace ne scelle rivières et lacs que 90 jour's en Gölaland, 150 jours en Svealand et 290 jours en Norrland. Son climat brusque et changeant tient à ce que cette région ne recevant pas les souflles modérateurs de l'Atlantique, obéit tantôt aux vents humides de l'Océan, tantôt aux vents continentaux de la Sibérie et de la Russie. Si d'une manière générale l'on peut dire que l'hiver y est très sec et 
y dure 6 mois, que l'été y est très court et l'automne plus ou moins nébuleux; les conditions changent au fur et à mesure yue l'on s'avance vers le nord et le nord-est. C'est ainsi que sur le Kalix l'hiver dure 9 mois. Dans la Laponie suédoise l'été comprend ce qu'on appelle le printemps dans d'autres pays, et l'automne; il se compose de 56 jour's qui offrent les caractères suivants : le 23 juin la neige fond, le $1^{\text {er }}$ juillet la neige a disparu, le 9 juillet les champs sont couverts de verdure; le 17, les plantes naturelles, semées ou cultivées, sont en pleine croissance; le 25 elles sont en floraison complète; le 2 août les fruits sont mûrs; le 10, les plantes laissent échapper leurs graines; le 18, la neige commence à tomber, et de cette époque au 23 juin de l'année suivante le sol est partout couvert de neige et de glace.

Si aux lignes isothermiques de l'année, qui oscillent diversement suivant les expositions du sol on combine les autres données du climat, abondance des pluies, force et direction des vents, ces lignes marquent alor's d'une manière générale les zones de vérjétation.

Nous disons générale, car on remarque des anomalies apparentes. C'est ainsi que bien que la température moyenne soit à tous les degrés de latitude plus élevée sur les côtes de la Norvège que sur le versant oriental du plateau, beaucoup d'espèces d'arbres se montrent beaucoup plus haut ver's le nord, en Suède qu'en Norvège. Ainsi, le sapin cesse de former des forêts en Norrège dès les frontières du Norrland, rers le cercle polaire; mais on le roit en Suède bien au nord de la même latitude. Pár un phénomène analogue, le bouleau qui s'arrête à 320 mètres sur les pentes norvégiennes, monte au doulble de la hauteur sur le rersant suédois. 
II.

\section{LES ZONES DE VÉGÉTATION}

Les chapitres précédents nous ont montré:

$1^{\circ}$ Que la région suédoise s'étend du sud au nord sur une longueur comprenant 14 degrés géographiques.

2 Q Qu'elle possèle de grandes plaines, de profondes vallées, des forèts immenses ou des Alpes couvertes de neiges et de glaces élermelles.

$3^{\circ}$ Que son substratum est constitué lantôt par les couches les plus anciennes du grlobe, tantôt par les plus récentes.

$4^{\circ}$ Que les maximum et minimum de la température de l'air et du sol y sont considérables. Nous ne serons donc pas étonnés de trouver dans sa végétation des diflérences profondes. C'est pour cela ru'il est bien difficile de donner à ce pays des rérgions végétales très distinctes.

Néanmoins étant donné :

$1^{\circ}$ La nature de la végétation,

$2^{\circ}$ L'altitude,

$3^{\circ}$ La hauteur polaire, on peut déjà distinguer trois régions principales, ce sont:

La Gothie, au climal assez doux et au reliel peu accentué. On y tronve des plaines immenses et certaines parties d'âge géologique récent possédant une végélation vigoureuse et riche analogue à celle dı Danemark et de l'Allemagne. On peut dire que c'est la zone du hétre.

Mais tandis que la pointe méridionale de ce tiers de la Suède se rattache à des pays plus méridionaux, sa partie supérieure présente deux éléments distincts : un à l'est et $n$ ì l'onest.dı haut plateau smálandais, ainsi que du lac Vetter. C'est dire que nous pouvons dans cette région élablir' des sous-régions dillérentes au point de vue de leur flore.

La deuxième région comprend le Svealand; le climat y est plus rude que dans la région précédente, mais moins froid que dans la région du Nord. C'est un pays de terrasses descendant vers les grands lacs ou s'abaissant des Alpes 
norrégiennes: saul la Néricie, son sol est constitué par des terrains primitils. Elle est parcourue par des chaines et des collines bien boisées laissant entre elles les vallées remarquables. Nous pouvons la caractériser d'un mot : c'est la zone du chíne. Il est à noter que le bassin du Mälar, constitué par des alluvions, possède la plus riche végétation de cette contrée.

La troisième région est le Norrland. C'est une zone spéciale particulièrement propice à la migration des végélaux. Chaque grand cours d'eau constitue une voie propre permettant aux espèces méridionales de remonter vers le nord ou aux esperces septentrionales d'émigrer vers le sud; de telle sorte fuc plus l'on s'élève vers le nord en suivant la côte ou à l'onest, vers les grandes $\Lambda$ Ipes, on suit pas à pas la disparition de la végétation sudo-européenne tandis que les types arctiques se montrent de plus en plus nombreux. C'est ainsi que l'aune glutineux est remplacé par l'aune blanchâtre, les conilères, d'abord alliès aux arbres à feuilles, deviennent bientùt prédominants, puis viennent le Prunus padus, le Sorbus aucuparia et le houleau; ces espèces cèdent à leur tour la place aux saules et aux osiers nains et l'on arrive dans la région alpine oì ne croissent que des herlies alpestres, des mousses chétives, des lichens qui indiquent la limite de la vie végétale.

Ces grandes divisions générales peuvent être complétées en faisant intervenir les associations régétales et leur distribution dans l'ordre climatérique; on peut dès lor's distinguer:

10 Zone du hêtre el du charme.

Les plus grandes forêts ou se distinguent cette essence sont en Scanie, Halland méridionale, Bleking et une partie du Smảland et du Bohuslïn. La limite ouest va donc jusqu'au $59^{\circ}$ degré tandis qu'à l'est, elle atteint le $57^{\circ} 5$ à la ville de Kalmar.

L'aire de dispersion du charme est sensiblement la même que celle du hêtre. Il se rencontre dans les provinces de Scanie et de Bleking, dans le Italland jusqu’à la Laga, 
dans le Smáland oriental où sa limile septentrionale est Vernamo. Il existe aussi dans l'ile d'Giland, mais non dans l'île de Gotland.

La zone du hêtre décrit une sorte d'are qui s'élève à l'ouest jusqu'au 59º degré et à l'est un peu au-dessus du $57^{\circ}$ degré ; la zone du charme peut être délimitée par une ligne droite traversant la Suède méridionale sous le $57^{\circ}$ degrée.

Parmi les arbres et les arbrisseaux qui se rencontrent dans ce groupe, on peut citer :

Ilex aruifolium, particulièrement abondant dans les parties méridionales de la Norvège.

Acer campestre, rabougri dans le S.-O. de la Scanie, très développé aux environs de Stockholm.

Sorlus aria, sur les côtes de Gotland, de la Norvègre, au N.-O. de la Scanie.

Cornus sanguinca, très abondant dans les provinces méridionales, surtout dans les îles d'CEland et de Gotland.

Ligustrum vulgare, cantonné dans les iles des côtes de Bohuslän.

Econyun curoparus. Sa limite coïncide à peu près avec celle du hêtre, bien qu'il soit développé d'une façon particulière dans la partie orientale du continent suédois.

Sambucus nigra, bien représenté dans les provinces du sud et de l'ouest, plus rare dans la Suède moyenne, se rencontre jusqu'à llemösand.

Lonicer C Periclymenum, sur les rocher's de la côte occidentale, de la Scanie an Bohuslïn pénètre dans les régions du Smáland, Vestrogothie et Dalsland.

Genista germanica au milieu des bruyères du centre du Halland.

Genista pilosa, dans la Scanie moyenne et septentrionale, dans la partie méridionale du Halland.

Genista tinctoria apparail dans plusieur's contrées de la Vestrogothie occidentale et clans la partie centrale du Halland. 
Coronilla Emerus, espèce mérilionale se rencontre en quelques points de la région cenlrale de l'île de Golland et sur les pentes calcaires de la côte orientale d'CEland.

II cliunthemum Fumana appartient à l'ile de Gotland.

IIclianthemum CElandicum cantonné dans l'île d'GEland.

Les Riubus sont nombrenx; les Rubus Radula, et thyprsoideus pridominent en Scanie, les R. pruinosus el glandulosus, dans le Smiland; les $R$. vulgaris et tacniarum, dans le Bohuslïn; $R$. horridus, au Skïrgàrd; les autres espèces anx formes multiples ont une aire de dispersion beaucoup plus large.

Le genre liosa est bien représenté; $R$. canina abonde dans la Suède moyenne, $R$. villosa est un type plus méridional, $R$. tomentosa se refrouve jusqu'à Helsingland, $R$. moilissima pénc̀tre jusqu'au Jemtland, enfin $R$. cinanomemsa fleuril sur les $A$ lpes de la Laponie.

Hedra helix alfectionue surtoul les provinces méridionales el s'élève jusqu'au $59^{\circ}$ de latilude.

Parmi les espèces herbacées, je citerai :

Ajuga genevensis L. (Scanie).

Acer campestre L. (Scanie).

Asine tenuifolia viscosa Schreb. (Scanic).

Astragalus arenarius L. (Scanie).

Astragalus lyypoglotlis L. (Scanie, Smäland).

Allium carinatum L. (Scanie).

Airopsis caryopliyllea Fr. (Scanic).

Aconitum cammarum L. (Smaland).

Arenaria gothica Fr. (ile de Golland, ingermanland).

Artemisia laciniata Wild. (île dowland).

Artemisia rupestris L. (ile de Golland et d'(oEland).

Anemone silvestris L. (ile de Golland et d'otiand).

Adonis rernalis L. (ile de Golland et d'okland).

Anacamptis pyramidalis Rich. (ile de Gotland el d'oEland).

Antirrhinum Oroutium L. (Bleking, Scanie, Gotlant el (Wiland).

Anthericum ramosum L. (Scanie, Gotland, (Elland).

Ajuga reptans L. (Smiland). 
Anthriscus vulgaris Pers. (Smailand). Alisma ranunculoides L. (Smàland).

Anthericum Liliago L. (Scanie, Bleking, OEland).

Allium montanum L. (Scanie-Dalsland).

Betonica officinalis L. (Scanic).

Batrachium Rionii Nym. (Golland).

Batracchium hederaclïum L. (Scanie, IIalland, Gotland, Bohuslän).

Bellis perennis L. (Smáland).

Bupleurum tenuissimum (Smàland).

Bromus racemosus L. (Smȧland).

Cineraria campestris Retz (Scanie).

Ceratopiiyllum submersum L. (Scanie).

Carex Boenninghanseniana eniania Weich (Seanie).

Cephalanthera pallens Rich. (Gotland).

Calamagrostis varia P. S. (Gotland).

Carex Schreberi Schrank (OEland, Upland).

Crepis virens L. (Gotland, OEland).

Coronilla Emerus I. (Gotland, OEland).

Carex tomentosa L. (Gotland, OEland).

Cyperus fuscus L. (Gotland, Scanie).

Circaa intermedia Ehrls. (Scanie, Halland).

Cotula coronipifolia L. (Bohuslän).

Cuscuta Ligustri Aresch (Bolıslän).

Cerastium tetrandrum Curt. (Bohuslïn).

Carex punctata Gand (Bobuslïn).

Calamagrostis acutillora D. C. (Bolıuslän).

Carduus acanthoïtes L. (Smàland).

Cirsium oleraceum Scop. (Smảland).

Cichorium Intybus L. (Smảland).

Centunculus minimus. L. Smaland, Vermland, Dalsland).

Cherophyllum temulum L. (Smàland).

Cerastium brachypetalum Desp. (Smáland).

Carpinus Betulus L. (Smảland).

Corynephorus canescens P. B. (Smàland).

Cineraria palustris L. (Scanie, Bleking, IIalland, Smaland, Vestrogothie).

Cerastium glutinosum Fr. (Scanie, Bleking, OEland, Gotland, Bobuslän).

Dipsacus pilosus L. (Scanie). 
Digitalis purpurea L. (Bohuslän).

Dianthus arenarius L. (Scanie, Bleking, Ilalland, Bohuslän).

Digitaria linearis Kroch. (Scanie, Italland).

Dianthus superlus I). (Scanie).

Euphrasia salisburgensis lunk (Golland).

Epipactis microphylla Sw. ((wiand).

Erigeron Mülleri Lund. (Bohuslän el Vermland).

Epilobium virgatum Fr. (Vermand).

Elymus europaus L. (Scanic, OEland, Smaland).

Falcaria Rivini Host (Seanie, Gotland).

Filago germanica l. (Scanie el Hallanıl).

Gagea an vensis Pers. (Scanie).

Genista tinctoria L. (Vestrogothie).

Gymnadenia odoratissima Bs. ¡Gotland, Vestrogothie et Ostrogothie).

Galium rotundifolia I. (Gotland et Orland).

Globularia vulgaris L. (Golland el (kiland).

Gypsophila fastigiata L. (Scanie, Gotland, (Eland, Dalécarlie).

Glaucium luteım L. (Bohusläı).

Gnaphalium luteo-album L. (Smảland).

Galium saxatile L. (Smảland).

Gentiana pneumonanthe L. (Smiland, Vermland, D)alsland).

Gagea spathacea Schu!t. (Smaland).

Geranium palustre L. (Scanie, Bohuslän, Vestrogothie).

Ifypericum tetraplerum Fr. (Scanie).

Hypericum pulchrum L. (Halland).

IIspericum humifusum L. (Smaland).

Hordeum murinum L. (Scanie).

Hordeum pratense IIuds. (Scanie).

Ifieracium hyperboreum Fr. (Gotland).

Hieracium Estivum Fr. (Bohuslän).

Ilieracium onosmoides Fr. (Bohuslän).

Jeliauthenum oelandicum Mill. ((N)land).

Ileliauthemum Fumana Mill. (Gotland).

Helichrysum arenarium J. C. (Smàlanu).

Hypocharis radicata I. (Smảland).

Hypochıris glabra L. (Smảand).

Helosciadium inundatum Kíoch. (Smáland). 
Holosteum umbellatum L. (Smàland).

Inula ensifolia L. (Gotland).

Inula britanica L. (Scanie, OEland, Golland, Smảland, Vestrogothic).

Juncus dilfusus Hopp. (Scanic, OEland, Gotland, Smaland, Vestrogothie).

Juncus glaucus Ehrh. (Scanie, Smảand).

Juncus maritimus Lam. (Scanic, OEland).

Juncus capitatus Weig. (Bleking, Smảland).

Köleria cristata L. (Scanie).

Kochia hirsuta Nolt. ((E)land).

Lcpturus incurvatus L. (Scanie).

Lolium festucaceum Lk. (Scanie).

Linosyris vulgaris Cass. ((Eland).

Linaria Elatine L. (Scanic, Gotland).

Ligustrum vulgare L. (Bohuslän).

Lunaria rediviva (Scanic, Bohuslän, Vestrogothic).

Medicago minima Lam. (Scanie).

Melilotus dentata W. K. (Scanic).

Melanosinapis communis Spenn. (Smảland).

Nasturtium oflicinale I. (Smàland).

Orobanche major L. (Scanie).

Orobanche minor Lult. (Hallanil).

Orchis laxillora Lam. (Gotland).

Orchis militaris L. (Scanie, Bleking, Orland).

Orchis ustulata L. (Scanie, Bleking, OEland).

Orchis Morio L. (Scanic, Bleking, (Eland).

Orchis mascula L. (Smàland, Vermland, Dalsland).

Oxytropis campestris D. C. (OEland, Smáland).

Oxytropis pilosa L. (Gotland, Smảland, Ostrogothie).

Ononis campestris Koch. (Golland).

Oenanthe fistulosa L. (Scanic, Blcking, Smaland, Bolıustän).

Petasites alba L. (Scanic).

Petasites spuria L. (Scanie).

Picris hieracioïdes L. (Scanie).

Primula elatior Jacr. (Scanie).

Potamogeton gracilis Fr. (Scanic).

Potamogeto fluitans Roth. (Scanie, Upland). 
Psamma lualtica R. S. (Scanie).

Pulsatilla patens Mill. (Gotland).

Potentilla fruticosa L. (1)iland).

Polentilla opaca L. (Ścanie, Halland).

Potentilla collina Wib. (Smaland).

potentilla procumbens. Sibth. (Smaland).

J'punella grandiflora L. (otiland, Gotland).

Echinochloa crus-galli L. Scanie, Halland).

Plıleum arenarium L. (Scanic, IIalland, OEland, Bohuslän).

Rumex conglomeratus Schreb. (Scanic).

liumex acutus L. (Seanie).

liumex palustris Sm. (Golland).

Rumex sanguineus L. (Smàland).

Ranunculus oplıioglossifolius. Vill, (Gotland).

Ranunculus illyricus L. ((Wland).

Riubus tarniarum Lind. (Bohuslän).

Rubus Radula Weilı. (Scanie, Bleking, Bohuslïn).

Radiola linoides liotlı. (Smàland).

Resela Luteola L. (Smàlaud).

Senecio erucirfolius L. (Scanie).

Senecio aquaticus Huds. (Scanie, Halland, Smảland, Bohuslän).

Sonchus palustris L. (Scanic).

Scabiosa suareolens Desf. (Scanie).

Solanum villosum Lam. (Scanie).

Stalice Behen Drej (Scanie).

Sagina apetala cisspitosa Mresch. (Scanic).

Sagina ciliata Fr. (Scanie).

Scandix Peclen L. (Gotland, OEland).

Sisymbrium supinum L. (Gotland, (Eland).

Salix rosmarinifolia L. (Gotland, (Eland).

Stenhammaria maritima licich. (Bohuslän).

Sedum anglicum L. (Bolıuslïn).

Stachys arrensis l. (Smaland).

scirpus multicaulis Lindl. (Simaland).

Sorbus Aria L. (Bohuslän, Scanie, Dalsland, Golland).

'Trifolium alpestre L. (Scanie).

Tolieldia calyculata Wbg. (Gotland).

Thlapsi perfoliatum L. ((wland, Uplanol). 


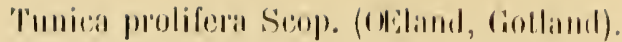

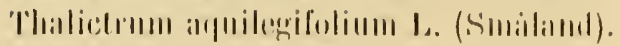

'Pesestalia mulicambis lir. (Smalame).

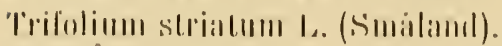

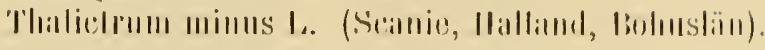

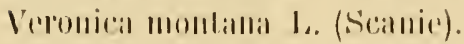

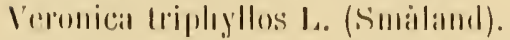

V'iolar prallensis M. Li. (Conllamb, Maland).

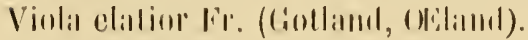

Valerianclla desulata Ponle. (Simalamal).

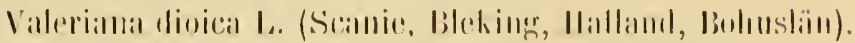

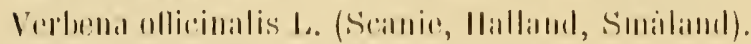

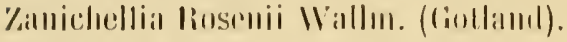

\%ostera minor Nolle. (Lohmslïm).

\section{$2^{\circ}$ ZONE DU CHENE}

In végralion de celle zone dillère nolahlenent de la précérlente; les espreces méridiomales y sont lont rances, limulis que les lypes de la flore seplemldomale y deviement. nommenx. Si colle zone possede la pluparl des andes i lenilles

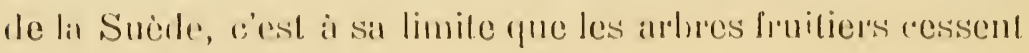
de monir leurs fruils.

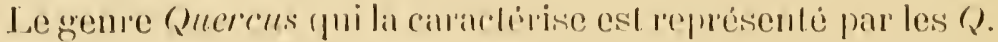

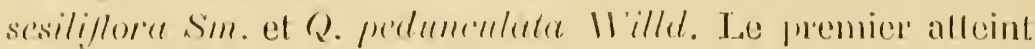

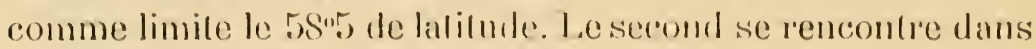
la parlie oredentale de la Néricie el daus le voisinagre du lac dälmar, juis dams le Vermand oceidenlal el suil il l'ouest

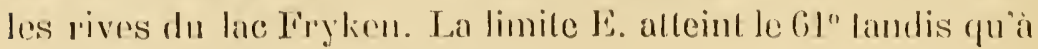
l'O. elle s'abaisse jusqu'an 60".

Ainsi la région du chêne s'blend sur lonte la Suède moyenne el la partic méridionale dn Normland.

l.es arbres à lemilles de celle zone sont:

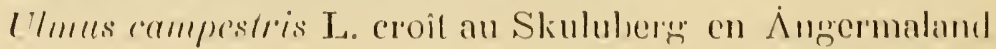
et dams la partic seplentrionale du Jemlland on il s'avante dans la région alpestre. 
Ulmus montana Sm Fr. a la même extension.

Alnus glutinosa Gaettn. a pour domaine la Suède moyenne et méridionale el s'avance vers le nord pour s'arrêter en Angermaland.

Sorbus scandica est caractéristique de notre végétation, commun en Suede moyenne, il s'avance au nord jusqu'à 625 , tandis que vers le sud, il semble s'arrêter à la Scanie septentrionale.

Fraxinus excelsior, dont la limite altudinale se confond avec celle du chêne $\left(61^{\circ}\right.$ lat. nord), s'ètend des provinces méridionales jusqu'au Helsingland et en Dalécarlie.

Ar' platanoüdes s'ètend de la Scanie jusque dans la partie moyenne du Vermland ainsi qu'en Néricie et dans le Helsingland; sa limite au nor'd peut être fixée à $63^{\circ} 5$ latitude.

Tilia parvifolia a la même extension que l'érable. Quant au Sorbus fennica, il préfère le climat maritime, aussi le trouve-t-on dans le Roslagen et à Södertürn.

Les saules et les osiers sont très répandus; ce grenre est, dans notre pays comme dans toutes les régions montagneuses et alpestres de l'Europe, représentépar des formes difficiles à déterminer. Une partie de ses représentants sont d'origine étrangère el lenr présence chez nous est due à ce qu'ils y ont été primitivement cultivés. Ce sont : le saule blanc (Salix alba), le sanle fragile (Salix fragilis), le saule des vanniers ou osier blanc (Salix ciminalis et le saule pourpré (Salix purpurea).

Les espèces suivantes: Berberis vulgaris, Corylus avellana, Taxus baccata, Viburnum opulus, Cotoneaster vulyaris, C. nigra, Prunus spinosa, Rhammus Frangula, R. cretharticus, Cratergus oxyacantha, Lonicera xylosteum, L. corulca, Erica Tetralix, croissent sous la forme d'arbrisseaux dans la région du chène.

On peut aussi dans cette zone établir plusieurs sousrégions caractérisées par les espéces végétales qui ont des limites trés nettes d'extension vers le nord. 
$1^{\circ}$ Plantes qui ne franchissent pas la chaîne montagneuse qui sépare l'Ostrogothie de la Sudermanie et de la Néricie:

Alchemilla Aphanes L.

Atriplex rosea $\mathrm{L}$.

Airopsis praecox Fr.

Avena strigosa $L$.

Cardamine silvatica $L$.

Camelina sativa $\mathrm{L}$.

Cornus sanguinea $\mathrm{L}$.

Corydalis cava Schw.

Filago minima Fr.

Geranium dissectum L.

Glyceria plicata Fr.

Hydrocotyle vulgaris $\mathrm{L}$.
Myosotis versicolor Fr.

Pulmonaria augustilolia L.

Rubus corylifolius Sm.

Scirpus setaceus $\mathbf{L}$.

Sium augustifolium L.

Stellaria Holostea L.

Teucrium Scordium L.

Vicia tenuifolia Roth.

Potamogeton polygonifolius Pourl'.

Quercus sessiliflora Sm.

2 - Plantes qui ne dépassent pas la région sud du lac Mälar :

Carex silvatica IIuds.

Dianthlis Armeria L.

Lirythraea Centaurium L.

Erica Tetralix L.

Iledera Helix L.

Holcus mollis L.

Hutchinsia petrea Br.

Lonicera Periclymemum L.

Lotus uliginosus Schk.

Melica uniflora L.

Pimpinella magna $L$.

\section{Environs de Stockholm :}

Anagallis arvensis $\mathrm{L}$.

Ballota ruderalis $\mathrm{L}$.

Bromus sterilis L.

Cerefolium sativum Bess.

Chenopodium murale $\mathbf{L}$.

Callitriche hamulata Kïüz.
Pulsatilla pratensis Mäll.

Rubus suberectus Ands.

Rubus plicatus Weih.

Rubus thyrsoideus Wimm.

Rubus discolor Weih.

Rubus nemorosus Hayn.

Scabiosa columbaria $\mathbf{L}$.

Sherardia arvensis L.

Sedum rupestre L.

Sempervivum tectorum L.

Tragopogon minor Fr.

Carex evoluta IIr.

Carex paniculata $\mathbf{L}$.

Galium syjlvestre Pall.

Geranium columbinum L.

Gagea stenopetala Reich.

Hypericum montanum L. 
Jasione montana L.

Pulygonum Bistorta L.

Rubus Wahlbergii Arrh.

limmer maritimus L.

Sambucus Ebulus L.

Thymus Chamedrys Fr.

Trifolium procumbens L.
Verbascum thapsilorme L..

Verbascum Lychuitis L.

Veronica polita Fr.

Veronica opaca $\mathrm{Fr}$.

Vicia dumetorum L.

Vicia cassubica L.

$3^{\circ}$ Plantes qui ne croissent pas au-delà du Dal-elf :

Anthemis Cotula L.

Arnica montana L.

Armeria elongata Ilofim.

Anemone ranuneuloiles L.

Alliaria ollicinalis And.

Alyssum calycinum L.

Allium Scorodoprasum L.

Allium arenarium $\mathrm{L}$.

Allium ursinum L.

Allium Schenoprasum L.

Acorus Calamus L.

Alopecurus agrestis $\mathrm{L}$.

Brassica Napus L.

Bryonia allsa $\mathrm{L}$.

Brachypodium pinnatum P. B.

Brachypod. gracile P. B.

Bromus erectus Ituds.

Bromus commutatus. Schrad.

Bromus asper Nurr.

Bromus tectorum L.

Cirsium acaule L.

Crepis biennis L.

Convolvulus sepium L.

Cuscuta Trigolii Bab.

Cynanchum Vincetoxicum Br.

Corydalis pumila IIost.

Cardamine impatiens I.

Cerastium arvense $\mathrm{L}$.
Cratogus Oxyacantha L.

Cephalanthera ensifolia Rich.

Carex montana L.

Carex remota L.

Carex divulsa Good.

Daucus Carota. L.

Dentaria bulbifera L.

Draba muralis L.

Epilobium hirsutum L.

Epilobium parvillorum Schreb.

Epilobium roseum Schreb.

Epilobium tetragonum L.

Euphorbia exigua L.

Fragaria collina L.

Festuca gigantea Vill.

Geranium pyrenaicum L.

Geranium lucidum L.

Geranium molle L.

Gypsophila muralis L.

Hieracleum auriculiforme Fr.

Heracleum sphondylium. L.

Hypericum hirsutum L.

Inula salicina L.

Lappa major Gaerth,

Leontodon hastilis L.

Lahlyrus tuberosus $\mathrm{L}$.

Lemna gibba L.

Irosotis hispida Schlecht. 
Mentha silvestris $\mathrm{L}$

Marrubium vulgare $\mathrm{I}$.

Melampyrum arvense L.

Malva silvestris L.

Malva Alcea L.

Malva moschata $\mathrm{L}$.

Medicago falcata $\mathrm{L}$.

Melilotus arvensis Wallr.

Mercurialis perennis L.

Nepeta Cataria L.

Nasturtium amphibium. L.

Nasturtium anceps Wbg.

Neslia paniculata Dess.

Orobus niger L.

Ononis hircina L.

Orchis sambucina L.

Petasitús oflicinalis Mônch.

Pedicularis silvatica $\mathrm{L}$.

Pulsatilla vulgaris Mill.

Papaver Argemone L.

Pyrus communis L.

Poterium Sanguisorba L.

Potentilla verna $L$.

Prunus spinosa $\mathrm{L}$.

Potamogeton crispus L.

Potamogeton acutifolius Lk.

\section{Plantes croissant à Gefle:}

Androsace septentrionalis L.

Avena llavescens $\mathbf{L}$.

Ballota futida Lam.

Carlina vulgaris $\mathrm{J}$.

Crategus monogyna Jacq.

Chenopodium urbicum I.

Chenopodium glaucum L.

Fragaria elatior I.

Hieracium pratense $\mathrm{L}$.

Lillosperm. officinale L.
Ranunculus arvensis L.

Rosa rubiginosa L.

Rosa Acharii Billb.

Rosa villosa $\mathrm{L}$.

Rumex maximus Schreb.

Rumex hydrolapathum Ifuds.

Senecio Jacobita I.

Serratula tinctoria L.

Scorzonera humilis L.

Sambucus nigra L.

Symphytum oflicinale L.

Solanum nigrum $\mathrm{L}$.

Selinum carvifolia L.

Sinapis alba $\mathbf{L}$.

Saxifraga tridactylites L.

Salix undulata Ehrb.

Salix viridis Fr.

Salix Smithiana Willı.

Salix purpurea L.

Setaria viridis P. B.

Torilis Anthriscus Gmel.

Valerianella olitoria Poll.

Viseum album L.

Verbascum philomoïles L.

Veronica spicata L.

Vicia lathyroides L.

Lathraea Squamaria L.

Lysimachia Nummul. L.

Malva rotundifolia L.

Nasturtium silvestre L.

Ncoltia Nidus Avis L.

Onopordon Acanth. í.

Odontites rubra Pers.

Papaver dubium L.

Potamogeton comipresens L.

Phleum Eoehmeri Wib. 
Rosa coriifolia Fr.

Rumex conspersus Hn.

Salix alba $L$.
Veronica hederifolia L.

Viola odorata L.

\section{ZONE DE L'AUNE BLANCHATRE ET DES CONIFĖRES OU RÉGION DES FORÊTS}

Au point de vue végétal on y retrouve nombre des espèces des régions précédentes, mais les espèces méridionales en sont exclues sauf en quelques points particulièment abrités.

$L ' A$ lnus incana $L$. caractérise toute la bordure du littoral sur une longueur de 60 à $\$ 0$ kilomètres. C'est ainsi qu'il occupe la moitié supérieure du Norrland et il se mèle ensuite à l'Alnus glutinosa (Ángermaland), non signalé dans le nord de l'Uppland et du Vestmaland. On le retrouve à l'ouest et au sud est de l'Ostrogothie et il s'avance jusqu'à Sandsjö et Vrảnghult en Smáland. D'autre part il pénètre en Laponie, se rapproche de la chaîne du Kjölen, traverse foutes les provinces occidentales et grane enfin les montagnes de la Vestrogothie centrale ct le IIalland.

Les Coniferres forment d'immenses forêts qui courrent presque le quart de la surface totale de la Suède.

La partie septentrionale, particulièrement le Norrland, est une région forestière très riche; on rencontre aussi des forêts plus ou moins considérables dans la Suède méridionale et moyenne.

Partout le pin est associé au sapin en proportions variables, suivant le sol. Cependant au pied de la chainne des HautesAlpes ces deux essences occupent des régions distinctes; le pin s'y montre de 90 à 120 mètres plus haut que le sapin, qui en général ne s'élève guère à plus de 300 mètres, Toutefois le contraire a également lieu; par exemple le sapin s'élève au-rlessus du pin dans les vallées chaudes et profondément encaissées de Qvickjock (Laponie), de Gillsnüle et de Sandsele (Laponie) ainsi que sur les Alpes du Jemtland et sur l'Áreskutan. 
La flore herbacée de la zone de l'Aune blanchâtre est représentée par :

Artemisia campestris L.

Anchusa officinalis L.

Aetlıusa Cynapium L.

Aegopodium Podagraria L.

Agrimonia Eupatoria L.

$\Lambda$ vena elatior L.

Adoxa moschatellina $\mathbf{L}$.

Astragalus glycyphyllus L.

Anthemis tinctoria $\mathrm{L}$.

Artemisia absinthum $\mathrm{L}$.

Asperula odorata L.

Acer platanoides L.

Anemone liepatica L.

Atriplex patula $\mathbf{L}$.

Alnus glutinosa L.

Allium oleraceum L.

A rena pratensis L.

Anthemis arvensis L.

Artemisia vulgaris $\mathrm{L}$.

Ajuga pyramidalis L.

Anemone nemorosa L.

Arabis Thaliana L.

Agrostemma Githago L.

Arenaria trinervia L.

Arenaria serpyllifolia $\mathbf{L}$.

Nlisma Plantago L.

Avena fatua $\mathrm{L}$.

Avena pulsescens I.

Aspera spica Venti L.

Batrachium circinatum L.

Berberis vulgaris $\mathrm{L}$.

Bunias orientalis L.
I Gestrikland.

Helsingland

Medelpad.

Ängermaland.

Laponie.

Helsingland. 
Bidlens cernua L.

Botryehium peltatum $\mathrm{Fr}$.

Barbarea vulgaris Br.

Bromus mollis $\mathrm{I}$.

Angrermaland.

Bulliarda aquatica L.

Betula verrucosa Ehrh.

Butomus umbellatus L.

Bromus arvensis L.

Briza media I.

Bidens tripartita L.

Centaurea scabiosa L.

Convolvulus arvensis $\mathbf{L}$.

Cynoglossum ollicinale $\mathrm{L}$.

Clinopodium rulgare L.

Coloneaster vulgaris Lind.

Chenopodium bonus Henricus I.

Chenopodium vulvaria L.

Chenopodium polyspermum L.

Carex lirita I.

Carex stricla Goot.

Carex disticlıa Good.

Crepis nraemorsa T'usch.

Conium matculatum $\mathrm{I}$.

Cerastium semidecandrum L.

Chenopodium rubrum L.

Convallaria multiflura L.

Carex riparia $L$.

Campanula Cervicaria L.

Campanula glomerita L.

Campanula Trachelium L.

Campanula latifolium $\mathrm{L}$.

Campanula rapunculoides L.

Chenopodium polyspermum L.

Carex paludosa I.

Cynosurus cristalus L.

Laponie.

Helsingland.

Gestrilitand.

Medelpad. 
Campanula percisifolia L.

Cuscuta Epilinum L.

Camelina foctida Fr.

Cerastıum riscosmm L.

Corylus Avellana L.

Ceratophyllum demersum $\mathrm{L}$.

Carex Hornschuchiana Hop.

Carex Ericelorum Poll.

Carex pilulifera $\mathrm{L}$.

Carex vulpina Good.

Catabrosa arjuatica L.

Àngermaland.

Chrysanthemum segetum L.

Chrysanthenum I eucanthemum L.

Centaurea Jacea L.

Centaurea cyanus L.

Circium lanceolatum Scop.

Circium arvense Scop).

Crepis tectorum L.

Cuscuta Europara L.

Calamintha Acinos Clairv.

Corydalıs fabacea L.

Camelna silvestris $\mathrm{Fr}$.

Calitriche autumnalis $\mathrm{L}$.

Convallaria majalis $L$.

Convallaria Polygonatum L.

Carex glauca Scop.

Carex stellulata L.

Carex teretiuscula Good.

Carex elongala $\mathrm{L}$.

Calamagrostis arundinacea $\mathrm{L}$.

Laponie.

Delphinium Consolida L.

| Ielsingland.

J)aba verna L.

Mraba nemorosa J.

Diantlıus deltoides $\mathrm{L}$.

Jactylis glomcrata $\mathrm{L}$.

Ángermaland. 
Echinospermum Lappula L.

Euphrasia gracilis Fr.

Euphorbia Esula I.

Helsingland.

Erysimum cheirantoides L.

Erodium Cicutarium L.

Euphorbia Helioscopia L.

Medelpad.

Eriophorum latifolium Roth.

Echium vulgare L.

Elatine Hydropiper L.

Ervum hirsutum $\mathrm{L}$.

Errum Pseudo-Cyperus L.

Ervum Paradoxa IV.

Ervum Pulicaris L.

Eupatorium cannabinum L.

Ervum tetraspermum L.

Euphorbia Peplus L.

Epipactis latifolia All.

Epipactis palustris L.

Fraxinus excelsior L.

Ficaria ranunculoides Mònch.

Farsetia incana Br.

Laponie.

barseti

Filago montana L.

fumaria oflicinalis. L.

Festuca elatior L.

Galeopsis Ladanum L.

Gentiana Amarella L.

Geranium pusillum L.

Geranium pratense $\mathrm{L}$.

Geum urbanum L.

Geum intermedium Ehrh.

Gagea lutea L.

Galium mollugo L.

Gestrikland.

Helsingland.

Laponie.

Helsingland. 
Geranium sanguineum L. Glyceria aquatica $\mathrm{L}$.

\} Medelpad.

Galium verum $L$.

Glechoma hederacea L.

Gentiana campestris L.

Gagea minima L.

Geranium Robertianum L.

Glyceria fluitans.

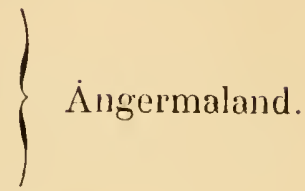

Herinania glabra L.

Hieracium cymosum L.

Hieracium setigerum Fr.

Heracleum sibiricum L.

Hypericum quadrangulum $L$.

Hypericum perforatum $\mathrm{L}$.

Humulus Lupulus L.

Holcus lanatus $\mathrm{L}$.

Hieracium Pilosella L.

Hieracium Auricula L.

Hieracium glomeratum Fr.

Hypochaeris maculata $\mathbf{L}$.

Hyoscyamus niger $\mathbf{L}$.

Herminium Monorchis L.

Laponie.

I Medelpad.

Hieracium stoloniflorum Kit.

Helianthemum vulgare L.

Hydrocharis morsus Ranae $\mathrm{L}$.

Impatiens Noli tangere L.

Iris Pseudo-Acorus L.

Juncus effusus L.

Juncus compressus L.

Laponie.

Leonurus Cardiaca L.

Libanotis montana All.

Lepidium campestre $\mathbf{L}$.

Ángermaland.

Laponie.

Helsingland.

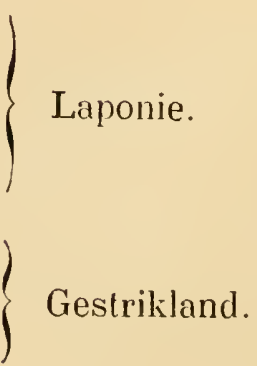


Lycopus europaeus L.

Lolium temulentum L.

\} Helsingland.

Linaria vulgaris $\mathbf{L}$.

Limaria minor L.

Lepidium ruclerale L.

Medelpad.

Lappa tomentosa Lam.

Lapsana communis $\mathrm{L}$.

Lonicera Xylosteum L.

Lamium album.

Lanium intermedium.

Lychnis Flos Cuculi L.

Latliyrus silvestris L.

Listera ovata L.

Lemna polyrrhiza L

Lolium linicola Sond.

\section{Angermaland.}

Lappa minor D. C.

Lactuca muralis L.

Lobelia Dortmanna L.

Lycopsis arvensis L.

Lithospermum arvense $L$.

Lamium purpureum L.

Lamium amplexicaule L.

Littorella lacustris L.

Linum catharticum L.

Lepigonum rubrum L.

Lythrum Salicaria L.

Lathyrus palustris L.

Latlyrus pratensis L.

Lotus corniculatus L.

Lemna minor L.

Lemna trisulca L.

Lolium perenne.

Melampyrum nemorosum L.

Malachium aquaticum Fr.

Mentha gentilis L.

Laponie. 
Melampyrum cristatum L.

Monotropa Hypopithys L.

\} Helsingland.

Malva borealis Wa'm.

Medicago lupulina L.

Melilotus officinalis. L.

Melilotus alla L.

Medelpad.

Mentha aquatica L.

Myosur'us minimus L.

Melandrium pratense Rôhl.

Myriophyllum verticillatum L.

Àngermaland.

Matricaria Chamomilla L.

Myosotis stricta Link.

Myosotis caspitosa Sch.

Mentha arvensis L.

Myrica Gale L.

Malaxis paludosa Sw.

Ophrys myodes L.

I Gestrikland.

Origanum vulgare L.

I Helsingland.

Ononis arvensis L.

I Medelpad.

Orobus vernus $L$.

Orobus luberosus L.

Laponie.

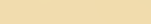


Polygala amara L.

Polygonum Persicaria L.

Potamogeton lucens L.

Medelpad.

Potamogeton zosterifolius Schum.

Primula veris $\mathrm{L}$.

Pyrola media Sw.

Potamogeton obtusifolius L.

Potamogeton pusillus L.

Ángermaland.

Primula farinosa $\mathrm{L}$.

Plantago media L.

Pimpinella Saxifraga L.

Peplis Portula L.

Potentilla Anserina L.

Polygonum mite. L.

Laponie.

Polygonum Iydropiper L.

Polygonum dumetorum L.

Platanthera bifolia L.

Potamogeton nitens Wib.

Quercus Robur L.

I Gestrikland.

Ranunculus Lingua L.

Ranunculus polyanthemos L.

Ranunculus bulbosus L.

Ribes Uva Crispa L.

Rhamnus cathartica L.

Rosa dumetorum Thuill.

Rosa mollisima Willd.

Rosa tomentosa Sm.

Rubus caesius L.

Rumex obtusifolius L.

Rhynchospora fusca Rœm. et Sch.

Helsingland.

Rhinanthus major Ehrb.

Raphanus Raphanistrum L.

Rosa canina L.

Rumex crispus L.

Ängermaland. 
Ranunculus sceleratus L.

Rhamnus Frangula L.

Ribes nigrum L.

Rhynchospora alba L.

Laponie.

Sanicula europaea L.

Silene noctillora $\mathrm{L}$.

Sedum sexangulare L.

Salix viminalis $L$.

Sturmia Loselii Rich.

Scirpus compressus L.

Sessleria corrulea And.

Symphytum orientale L.

Sisymbrium oflicinale L.

Saxifraga granulata L.

Sedum album L.

Salix fragilis L.

Stratoites aloides L.

Solanum Dulcamara L.

Senebiera Coronopus L.

Stellaria uliginosa L.

Sorbus scandia L.

Scleranthus perennis L.

Gestrikland.

Helsingland.

Senecio viscosus L.

Senecio silvaticus L.

Stachys silvatica. L.

Stachys palustris I.

Scrofularia nodtosa L.

Stellaria palustris Retz.

Spergula Morisonii Bor.

Sedum Thelephium L.

Sparganium ramosum Huds.

Sparganium natans L.

Medelpad.

Senecio vulgaris $L$.

Sonchus arvensis L.

$\{$ Laponie.

Ángermaland. 
Sonchus oleraceus L.

Sonchus asper L.

Succisa pratensis Mònch.

Sium latifolium L.

Sisymbrium Sophia L.

Silene nutans L.

Sedum acre L.

Spirza Filipenılula L.

Scleranthus annuus L.

Salix cinerea L.

Salix aurita L.

Salix repens. L.

Sagittaria sagittifolia L.

Sparganium simplex Nuds.

Sparganium minimun $\mathrm{Fr}$.

Scirpus pauciflorus Ehrh.

Scirpus acicularis L.

Scirpus silvaticus L.

Taxus baccata I.

I Gestrikland.

Trifolium fragiferum L.

I Medelpad.

Trichera arvensis L.

Thymus Serpyllum L.

Tilia parvifolia Ehrh.

Trifolium agrarium L.

Trifolium spadiceum L.

Trifolium medium L.

Tragopogon pratensis L.

Trifolium arvense $\mathbf{L}$.

Trifolium montanum L.

Typha augustifolia $\mathrm{L}_{\text {. }}$

Triodia decumbens $\mathrm{L}$

Tanacetum vulgare L.

Thalictrum flavum $\mathbf{I}$.

Thalictrum simplex L.

Laponie.

Angermaland.

Helsingland.

Laponie. 
Trifolium hybridum L.

Typha latifolia L.

\} Laponie.

Ulmus montana Sm.

| Ảngermaland.

Verbascum nigrum L.

Viola hirta L.

\} Medelpad.

Verbascum Thapsus L.

Veronica Beccabunga L.

Veronica arvensis $\mathrm{L}$.

Veronica verna L.

Veronica agrestis $\mathrm{L}$.

Viola stagnina kit.

Viscaria vulgaris Röht.

Vicia villosa Rïth.

Vicia sepium L.

Vicia augustifolia Ròth.

Viburnum Opulus L.

Valeriana sambucifolia Mit.

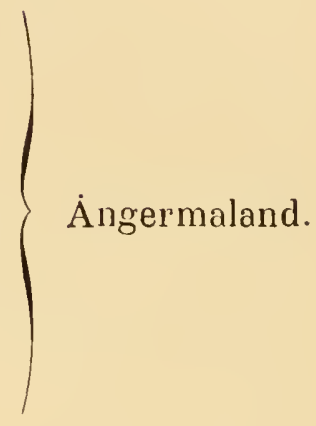

I Helsingland.

La flore herbacée de la zone des conifères peut ètre divisée en deux régions:

$1^{\circ}$ La région sylvatique inférieure, s'arrètant à la limite inférieure du sapin et comprenant:

Bromus secalinus L.

Baldingera arundinacea Dum.

Cirsium palustre Scop.

Cicuta virosa $\mathrm{L}$.

Carex acula L.

Carex leporina L.

Gnaphalium silvaticum L.

Hieracium falax W.

Lysimachia vulgaris $\mathrm{L}$.

Lysimachia thyrsillora L.
Myosotis arvensis llollm.

Myriophyllum alternillorum D.C.

Nymphea alba L.

Plantago lanceolata L.

Polygonum amphibium L.

Potamogeton natans $L$.

Potamogeton rufescens schrad.

Potamogeton praelongus Wulf.

Phleum pratense L.

Ranunculus auricomus L. 
Scutellaria galericulata L.

Sagina nodlosa Fen\%l.

Scirpus lacustris $\mathrm{L}$.

$2^{\circ}$ La rézion sylvatique supérieure au-dessus de la limite du sapin comprenant :

Achillea Millelolium L.

Asperugo procumbens L.

Angelica silvestris $\mathrm{L}$.

Alopecurus geniculatus $\mathrm{L}$.

Batrachium heteropliyllum L.

Brassica campestris L.

Barliarca stricta Andr.

Carduns crispus L.

Carum Carvi L.

Chelidnnium majus L.

Cansella bursa pastoris L.

Circila alpina L.

Calluna vulgaris Lalieb.

Chenopodium rubrum $\mathrm{L}$.

Callitriche verna $\mathrm{L}$.

Corallorliza innata L.

Cal'a palustris L.

Carex filiformis L.

Carex limosa L.

Carex panicea L.

Carex ornilhopoda L.

Carex rulgaris frr.

Carex juncella Fr.

Carex cilespilosa L.

Carex canescens L.

Carex chordorhiza Ehrh.

Calamagrostis Epigejos L.

Calamagrostis lanceolata Rotlı.

Drosera rotundifolia L.

Droscra longilolia L.

Drosera intermedia, Hay.

Erigeron acris L.

Epilobium palustre L.
Turritis glabra L.

Viola palustris L.
Eriopho:um polystachium L.

Eriophor. gracile lioch.

Galium boreale L.

Galium palustre L.

Galium uliginosum L.

Galium Aparine L.

Galeopsis Tetraluit L.

Galeopsis versicolor L.

Goodeyra repens I.

Hieracium caesium Fr.

Hieracium Saxifragum Fr.

Ilieracium tridentalum Fr.

Ilieracium umbellatum L.

Ilieracium paludosum L.

Ilippuris vulgaris L.

Juncus balticus Wild.

Juncus articulatus L.

Jincus alpinus Wilı.

Juncus bufonius L.

Limosella aquatica $\mathrm{L}$.

Listera cordata L.

Luzula pilosa L.

Myosolis palustris L.

Menyanthes trifoliata L.

Myriophyllum spicatum L.

Montia fontana L.

Majanthemum bifolium L.

Molinia coreulea L.

Nuphar luteum L.

Nasturtium palustre D. C.

Oxalis Acelosella L.

Oxycoccus palustris Pers.

Orchis maculata L. 
Orchis augustifolia L.

Prunella vulgaris L.

Pedicularis palustris L.

Plantago major L.

Pensedanum palustre L.

Pyrola rotundifolia $\mathrm{L}$.

Pyrola minor L.

Pyrola secunda L.

Pyrola unillora $\mathrm{L}$.

Polygonum aviculare $\mathrm{L}$.

Polygonum lapatifolium L.

Polygonum Convolvulus L.

Pinus silvestris L.

Pinus Abies L.

Poa serotina Ehrh.

Poa annua L.

Phragmites communis. Trin.

Ranunculus Flammula L.

Ranunculus reptans L.

Rubus idaeus L.

Rumex domesticus II.
Sinapis arvensis L.

Subularia arquatica L.

Silene inflata L.

Sagina procumbens L.

Spiraea Ulmaria L.

Salix pentandra L.

Scirpus palustris $\mathrm{L}$.

Thlapsi arvense $L$.

Trifolium repens $\mathrm{L}$.

Trifolium pratense L.

Triglochin palustre L.

Triticum repens $\mathrm{L}$.

Utricularia intermedia llay.

Utricularia vulgaris $L$.

Utricularia minor $\mathrm{L}$.

Urtica urens L.

Urtica dioica L.

Veronica scutellata L.

Viola canina $L$.

Vaccinium Vitis Ldera L.

\section{$4^{\circ}$ ZONE DU BOULEAU}

Le bouleau (Betula alba, B. verrucosa, B. pubescens, B. glutinosa) est très abondant depuis la Scanie moyenne et septentrionale jusqu'à l'extrême nord de la Suède; il forme une zone bien nette et assez régulièrement conlinue audessus de la région du pin el du sapin; au fur et à mesure que l'on s'élève dans celte zone on remarque qu'il diminue de taille, et devient un petit arbrisseau rampant et rabougri aux dernières limites de la végétation.

Quant au Betula nana on le rencontre dans les marais du Norrland, dans le Halland et Bohusläı, landis que sa limite méridionale est Skatelöf sur le lıaut platean smalandais; il s'èlève dans les plaines centrales des Alpes scandinaves jusqu'à 150 mètres.

D’après M. le professeur Friss, le Myrica gale, très abon- 
dant dans les provinces moyennes du Norrland et dont l'aire de dispersion s'étend de la Scanie jusqua au nord de la Botnie, formerait me région propre. C'est à la latitude du $6 \mathfrak{1}^{\circ}$ que se place la limite entre la llore européenne et la llore arctique ; dans les 3 zones précédentes se rencontre toute une série interressante d'espéces; ce sont:

Le Juniperus rommunis, qui s'étend de la Scanie aux Ilautes- $\Lambda$ lpes, taudis qu’il acquiert son maximum de développement dans les régions méridionales; sa taille diminue dans les régions froides, pour se présenter' sous la forme d'un arlorisseau rampant au sommet des Alpes.

Les Salir; les S.pentandra, caprea, nigricans, ont une aire de dispersion qui s'étend de la région des bouleaux jusqu'en Scanie; les S. hastata et $S$. cagans descendent moins ver's le sud; les $S$. cinerea, aurita, repens, pénètrent jusque dans la Bolnie septentrionale. Citons encore S. daplinoïdes, $S$. triandra, cantonnés le long des cours d'eau et à l'embou. chure des grands lleures.

Le Sorbus aucuparia se rencontre dans toute l'étendue de la Suède; il clépasse la zone du bouleau et s'élève jusqu'à 600 mètres d'altitude.

Le Prunus padus accompagne l'espèce précédente, mais il ne s'élève pas au-delì de la région du bouleau.

Le Populus tremula s'arance jusquà la limite supérieure du pin.

\section{Citons encore:}

Daphus Mezereum L.

Riulus ildulus L.

Riulums saxatilis L.

Rubus chamaenorus L.

Rubus arcticus L.

Ribes alpinum $\mathrm{L}$.

Rilues nigrum L.

Ribes rubrum L.
Cailuna rulgaris $\mathrm{L}$.

Vaccinium uliginosaum L.

Vaccinium oxycoccus $\mathrm{L}$.

Vaccinium vitis Idaea L.

Vaccinium Myrtillus L.

Arctostaphylos Uva-Ursi Spreng.

Myricaria germanica Desr.

Hippophae rhamnoides L. 
La flore herbacée de la zone du bouleau ou région subalpine possède:

Antennaria dioica L.

Actiea spicata L.

Arabis hirsuta Scop.

Anthyllis vulneraria L.

Aretostaphylus Uva Ursi L.

Agrostis stolonifera L.

Agrostis vulgaris $L$.

Agrostis canina L.

Alopecurus pratensis L.

Cerefolium silvestre Bess.

Cerastium vulgatum L.

Carex irrigua $\mathrm{Wbg}$.

Carex llava L.

Carex Oederi Ehrh.

Carex pallescens L.

Carex dioica L.

Daphne Mezereum L.

Epilobium angustifolium L.

Epilobium montanum L.

Fragaria vesea $\mathrm{L}$.

Gnaphalium uliginosum L.

Geum rivale L.

Gymnadenia Conopsea L.

Hicracium murorum L.

Hieracium vulgatum L.

Hieroclıloa horealis L.

Juncus supinus L.

Linnea borealis L.

Myosotis silvatica IIoffm.

Melampyrum pratense L.
Melampyrum silvaticum L.

Melica nutans L.

Milium effusum L.

Pinguicula vulginis $\mathrm{L}$.

Potentilla argentea L.

Potentilla Tormentilla L

Prunus Palus L.

Populus tremula L.

Paris quadrifolia L.

Poa sudetica Haencke.

Poa trivialis L.

Rhinantus minor Ehrlt.

Ribes rubrum L.

Rosa cinnamomea L.

Rubus saxatilis L.

Stellaria nemorum L.

Stelliria media L.

Stellaria graminea L.

Stelleria Friesiana D. C.

Sedum anmuum L.

Sorbus Aucuparia L.

Salix caprea L.

'Tussilago Farfara L.

Taraxacum ollicinale Wigg.

Triticuu caninum L.

Valeriana ollicinalis L.

Veronica serpyllifolia L.

Veronica oflicinalis L.

Viola mirabilis L.

Viola montana L.

\section{$5^{\circ}$ LA ZONE ALPINE}

Cette zone débute an-dessus de la limite des arbres. Elle est constituée à sa partie inférieure par des lourrés de saules. On y distingue particulièrement $S$. pliylicifolia et S. Lappo- 
num, S. glauca, S. lanata. Le premier parvient it ses plus grandes dimensions dans la région forestière et descend jusqu'au Vermland ; les aulres ont leur principal développement dans les vallées; le S. lapponum descend à peine andessous de l'Uppand, le S.glanca s'avance jusrfu'en Dalécarlie et en Vermland, mais à l'est il ne dépasse pas l'Ángermaland et le $S$. lanata ne va que jusque dans le Herjedal, sans même s'éloigner de la région alpestre.

La camarine à lruits noirs (Empetrum nigrum, en suédois krakris) s'élève dans la région alpestre jusquä la limile des neiges et se trouve partout jusqu'en Scanie. S. myrsinites, bien que caractéristique de la région alpine, descend dans la région forestière ainsi que $S$. Arbuscula.

Quant au S. ovata, S. polaris, S. herbacea et S. retirulata, ils sont caractéristique des haules régrions alpestres.

Les représentants herbacés de la végèlation de cetle zone sont :

Alchenilla vulgaris $L$.

Aira caespitosa L.

Aira flexuosa L.

Anthoxanthum odoratnm L.

Campanula rotundifolia L.

Cornus suecica L.

Coeloglossum viride IIn.

Carex vesicaria $L$.

Carex ampullacea $\mathrm{L}$.

Carex capillaris L.

Calamagrostis stricta Ehri.

Euphrasia officinalis L.

Empetruı nigrum L.

Festuca rubra L.

Festuca ovina L.

Geranium silvaticum $L$.

Gymmadenia albida Br.
Juniperus communis $\mathrm{L}$.

Leontodon autumnate $\mathrm{J}$.

Luzula campestris L.

Melandrium sylvestre Röhl.

Parnassia palustris L.

Potentilla maculatia Pourr.

Polygonum viviparum $L$.

Potamogeton gramineus L.

Poa pratensis L.

Poa nemoralis L.

Ranunculus acris $\mathrm{L}$.

Rubus Chamaemorus L.

Rumex Acetosa L.

Rumex acelosella L.

Solidago Virgaurea L.

Trientalis europaea L.

Dans la partie supérieure de celte région, la flore n'est représentée que par quelques lichens et diverses mousses. 


\section{$6^{\circ}$ ZONE ARCTIQUE}

Les hautes montagnes couverles de glacier's de la Scandinarie doment asile ì foule me population vémélale rui apparlient an domaine arelique. On peul les diviser de la façọn suirante:

a) Espèces exclusivement alpestres:

Arenaria humifusa Wlog. (Laponie de Lule).

Antennaria carpathica Wlog. (Laponie de Lule el de Torne).

Arnica alpina Mnrr. (Laponie de Lule, de Torne et de Pite).

Andromeda tetragona L. (Laponie de Lule, de Torne et de Pite).

Antemaria alpina $\mathrm{L}$. (Ilerjedal).

Arabis alpina L. (Ilerjedal).

Alsine lifllora L. (Herjedal).

Alsine stricla Wlog. (IIerjedal).

Andromeda hypnoides L. (Herjedal).

Aira alpina L. (Ilerjedal).

Cardamine hellidifolia L. (Ilerjedal).

Chamacorchis alpina L. (Herjedal).

Carex ustulata IIoppe. (Iterjedal).

Carex laxa Wlog. (Herjedal)-

Carex rarillora Sm. (IIerjedal).

Carex pedata Wlog.

Cares atrata L. (Herjedal).

Carex pulla Good. (Iler jedal).

Carex lagopina Wbog. (Ilerjedal).

Carex rupestris All. (Herjelal).

Carex microglochin Wlog. (Herjedal).

Carex rigida govol (I balécarlie).

Cerastum trigynum Will. (I)alícarlic).

Draba Wahleubergii IIn. (Laponie de Lule el de Pite).

Ilianensia lapponiea $\mathrm{L}$. (Herjedal).

Draba nivalis Lillj.

Iraba hirta L. (Ilerjerlal).

Iraba alpina L. (Herjedal).

Dryas octopetala L. (Ilerjedal).

Lrigeron elongatus Ledeb. (Ilerjedal).

Erigeron alpinus L. (IIerjedal). 
Erigeron muillorus L. (Iterjedal).

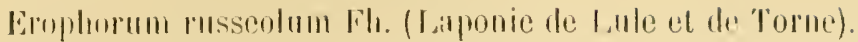

(inaphalium Smpinmm l. (Herjedal).

Gentiana tenella Roth (Iterjedal).

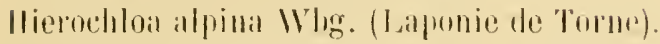

Ilicracimm argentemm Fr. (1 apronic de Lale).

Heracium alratum I. (Herjedal).

Ilicracium alpiumm I. (Daléantice).

Juneus areticus L. (Herjerlal).

Juncus bighlumis L. (Ilerjed,Il).

Juncus triglumis L. (Herjedal).

Juncus trilidus L. (Herjedal el Dalécarlic).

Juneus castaneus Sm. (Herjedal el Jemlland).

lirnigia islandiea L. (Laponie de Lule ed de Pite).

I.uzula hyperborea Br. (Laponie de Lule).

Lazula Wahlenhergii IIr. (I aponic el Jemtlamb).

lazula arenata $\mathrm{Wrg}$. (Herjedal).

Lazula spicata L. (Dalécarlie).

Melandrium apfine lahl. (Lapunie le Torne).

Melandrium aprelalum (Laponie de Torne el Lule).

Oxytropis lapponira Gaud (Iterjedal),

l'apacer malicanle L. (Laponie de T'orno).

I'ealicularis hirsula L. (Laponic ale Torne, Lule et l'ite).

l'caliculriris flammé (Laponie de Torme, lule el l'itc).

Potentilla nivea L. (Lilponie de Torne, Lule et Pite).

Primula scotica llort (Herjulal).

I'rimula stricta IIornem (Herjedal).

Poa lasa IIrulie (Herjedal).

l'oa caesia Sm. (Dalécarlic).

Ply'ilorloce cerpulea L. (I)alícardie).

P'elicularis lapponiea L. (Daiécarlie).

Phara lrigidal L. (Laponie de Torne et de Lule el Herjedal).

Rhododendron lapponicum L. (Iapunic le Torne et de Lule et de pite.

Ramunculus nivalis L. (I aponnic et Jemtlaml).

lianunculus gracialis L. (IIejjedal).

liumex Acetosa alpinus 1. (Iterjedal).

lianunculus piggmas IVlug. (Dalícarlic).

Salix sarmentacea Fr. (Laponic de Lule). 
Saxifraga Cotyledon L. (Laponie de Lule el de Torne).

Saxifraga oppesitifolia L. (Herjedal).

Saxifraga cernua L. (IIerjedal).

Sibbaldia procumbens L. (Herjedal).

Salix myrsinites I. (Ilerjedal).

Salix arlıuscula I. (Ilerjerlal).

Salix orala sir. (Herjedal).

Salix polaris L. (Ilerjedal).

Salix reliculata L. (Herjedal).

Saxilraga stellaris L. (Dalécarlie).

Saxifraga aizoides J. (Dalécallie).

Saxiflaga rivularis L. (Jalécarlie).

Salix herbacea L. (Dalécarlie).

Triselum agroslideum Fr. (Lapronie de Torne).

Triticum violaceum Ilorn. (Herjerlal).

Triselum sulsspicatum L. (Herjedal).

Veronica alpina J. (Herjelal).

Veronica saxatilis L. (Herjelal).

Valılodea atropurpurea Fr. (Ilerjedal).

b) Espéces qui ont les $A$ pes comme limite supérieure et les provinces d’Ängermaland, Medelpad, IIelsingland, Ges triklaml, Vermand, Uppland, Oslrogollie, Vestrogollie, Smaland, Golland, Giland, Bohuslïn, Halland et Blekingr comme limile inféricure:

Lerostis borealis IIn (ingermalanil).

Astragalus alpitan I. (Ilelsimglamu).

Alnus puhescens Tausch. (Gestrikland).

Aconitun septentrionale I. (Upplant).

Anns incinla J. (Sunilimal).

Mchemilla alpina L. (Bohuslïn).

Batrachium confervoides Fr. (Gestrikland).

Bartsia alpina I. (Ostrogollie).

Betula intermedia Thom. (Smbiland).

BeLula nana L. (Smaland).

Carex Vahlii Sr. (Mealelpal).

Calamagrostis lapponicat Wlog. (Medelpad).

Carex tenuillora W'log. (Helsingland). 
Carex capitata I. (Helsingland).

Carex tenella Sch. (Vermland).

Cerastium alpinum L. (Vestrogothic).

Carex globularis L. (Smiland).

Carex vililis Fr. (Smilanol).

Carex loliacea I. (Smiland).

Calamagrostis phragmitoides $11 \mathrm{~m}$. (Smàland).

Calamagrostis Ilalleriana D. C. (Smaland).

Carer ayuatilis W'bg. (Bleking).

Epilobium lincare Nö̈hl. (Ingermaland).

Eriophorum callichrix Cham. (Ingermaland).

Epilobium alpimum L. (Melelpad).

Epilohium origanifolium Lam. (Helsingland).

Eivohorum capitatum IIost. (IIelsingland).

Eriophorum alpinum L. (Vermland).

Echinospermum deflexum Wbog (Smaland).

Erysimum hieracifolium L. (Smaland).

(Wrigeron Mülleri Lumd. (Bohuslïn).

Gnaplıalium norvegicum Gumn. (ingermalanı).

Galium trillorum Michx (Ilelsingland et Vermland).

Gialium trifidum L. (Smiland).

IIieracium crocatum Fr. (Medelpad).

Ilieracium corymbos Fr. (Ilelsingland).

Ilieraciuni prenanthoïdes Vill. (Hetsingland).

Juneus stygius L. (Smaland).

Mulgedium sibiricum Less (Melelpad).

Mulgedium alpinum Less. (Ilelsingland et Vermland).

Rubus castorcus L. (He!singland et Vermland).

Rubus arcticus I. (Upplanel).

Rihodiola rosea L. (Bohuslän).

Stellaria borealis Big. (ingrermaland).

Sagina saxatilis Wimm. (Medelpad).

Saussurea alpina D. C. (IFelsingland).

Spasganium hyperboreum Laestad. (Helsingland).

Sparganium afline Schnizl. (Vermland).

Sparganium lluitians Fr. (Vermland).

Sparganium oligo carpon ingstr. (Vermland).

Sceptrum carolinum L. (Smiland). 
Silene rupestris I. (Smiland).

Salix myrtilloides L. (Smailand).

Thalictrum alpinum L. (Angermaland).

Thalictrum rarillorum Fr. (Medelpad).

Tofieldia borealis L. (Helsingland et Vermland).

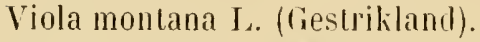

Viscaria alpina L. ((Eland, Bleling).

c) Espèces qui descendent jusque dans les régions les plus méridionales:

Andromeda polifolia $\mathrm{I}_{\text {. }}$

Betula odorata Bechst.

Cirsium heterophyllum L.

Cornus suecica $l_{1}$.

Chrysosplenium alternilolium L.

Carex vaginata Tausch.

Carex irrigua Sm.

Carex Buxbaumii L.

Carex microstachya Ehrlo.

Calmagrostis stricta Ehrh.

Eriophorum alpinum L.

Juncus tiliformis L.

Linnea borealis L.

Ledum palustre $\mathrm{L}_{\text {. }}$.
Pulsatilla vernalis Mtill. Polygonum riviparum L. Riubus Chamaemorus L. Saxifraga IIircu\}us L.

Salix vagans $A$ nd.

Salix hastata $\mathrm{L}$.

Salix nigricans Sm.

Salix triandra L.

Scheuchzcria palustris $\mathrm{L}_{4}$.

selunus ferrugineus $\mathrm{L}$.

Scirpus caespilosus L.

Trollius europacus L.

Viola suecica Fr.

Les espèces caractiristiques de la flore aretique sont en italique.

\section{FLORULE MARITIME}

$1^{\circ}$ De la rérion du clucine et du hitre.

Sur les deux cótes:

Artemisia maritima L.

Cuscuta haloplyyta Fr.

Cochlearia officinalis $\mathrm{L}$.

Eryngium marilimum L.

Lepidium latifolium L.

Lepigonum marinum Wbg.
Planlago Coronopus I.

Sagina subulata Presl.

Schoberia marilima Nley.

Triticum junceum $\mathrm{I}$.

Triticum hebestachyum Fr.

Zanichellia major Bönn. 
Sur la còte orientale (mer Baltique):

Bupleurmm tenuissimum $\mathrm{L}$.

Juncus maritimus Lam.

líochia hirsuta Nolte.

Psamma baltica R. S.
Sonchus palustris $\mathrm{L}$.

Scirpus carinalus Imur.

Zanichellia Rosenii Wallm.

Sur la cote occidentale (Kattegat et Skagerrak) :

Armeria maritima IVilı.

Batrachium hederaceum L.

Crambe maritima $\mathrm{I}$.

Cerastium tetrandrum Curt.

Carex punclata Gaumb.

Carex maritima Nü̈ll.

Carex hu'malulepis Drej.

Carex incurva Liglul.
Glaucium luteum I.

Ilaloscias scoticum Fr.

Stenhammaria maritima Rechl.

Statice Bielien I)rej.

Statice rarillora Drej.

Sedum anglicum I.

Rubus taeniarmm lind.

Zostera minor Nolte.

2o De la région du chêne.

Sur les deux coites:

Atriplex rosea L.

Atriplex litoralis L.

Caliile maritima $\mathrm{L}$.

Cochlearia danica $\mathrm{L}$.

Carex distans L.

Carex arenaria $\mathrm{L}$.

Erytliraea litoralis Fr.

Erythraea pulchella sir.

Glyceria maritima Wahlb.
Halimus peduneulatus Wallr.

Psanma arenaria R. S.

Sagina stricla L.

Salsola liali L.

Scirpus rufus Schrad.

Tripleurospermum maritimum L.

Zostera marina L.

Zostera angustifolia Nolte.

\section{Sur les côtes de la mer Raltique :}

Carex extensa Good.

Isalis tinctoria $\mathrm{L.}$.

Scutellaria lıastifolia L.
Samolus Valerandi L.

Silene viscosa L.

Espéces maritimes de l'extrême nord de la Suède:

Angelica litoralis Fr.

Atriplex lastata L.
Carex norregica Wild.

Elymus arenarius L. 
Festuca arundinacea Schr.

Glaux maritima L.

Ialiantluus peploides L.

Lepigonum salinum $\mathrm{Fr}$.

Lathyrus maritinus L.

Plantago maritima L.

Potamogeton marinus L.

Côtes de la Baltique:

Aira bottnica Wbg.

Batrachium marinum Fr.

Carex glareosa Wbg.
Senebiera didyma Pers.

Silene maritima Fr.

Stellaria crassifolia Ehrlt.

Salicornia herbacea L.

Scirpus maritimus L.

Triglochin maritimum $\mathrm{L}$.

Hippophaï rhamnoides L.

Zanichellia polycarpa Nolte. 


\title{
DEUXIÈME PARTIE
}

\section{LE MASSIF GENTRAL}

\author{
I. \\ GÉOLOGIE - OROGRAPHIE \\ II YUROGRAPHIE - CLIMATOLOGIE
}

Les limiles que l'on doit assigner an Massif Central résultent de sa constitution géologique: compris entre le 4.4 et le $47^{\circ}$ de latitude Nord, sa longitude est déterminée par le méridien de Paris, qui le traverse en laissant a l'list la majeure partie de son territoire.

Sa forme générale se rapproche de celle d'un triangle. Le côté le plus long orienté N. N. F., part de la dépression de Naurouze entre la Monlagne Noire el les Corbières, ra aboulir an col de Longpendu, entre les monts du Charolais et cenx de la Côte-d'Or et sépare le Massif Central du bassin du Phòne. Un autre côté E. N. E., part de l'extrémitè sep̧tentrionale du Morran, coupe presque à angle droit la Loire et la plupart de ses allutents de la rive gauche, va se terminer vers la rencontre de la Vienne et sépare le Massif Central des grandes plaines du hassin de la Loire. Le troisième côté part des environs de Montmorillon el va au col de Naurouze en délimitant les parties basses dı bassin de la Garonne. Les trois sommets de ce triangle correspondent it trois dépressions ou détroits permellant le passage d'un grand bassin hydrographique ì un autre, le détroit de Dijon, celui de Poitou et celui de Naurouze. En même temps ces 
dépressions isolent le Massif Central des Massifs roisins : les Tosges, la Brelagne el les Pyrénées (1).

C'est dire que l'on doit limiter le Massif Central anx terrains secondaires qui l'entonrent de tontes parts ot mettent en relief les sinnositis de son contonr : caps de terrains primitils ou golles secondaires.

\section{GEOLOGIE}

Ere primaire : Le Massil Central de la France apparait comme une ile primitive se dressant dans la grande mer primordiale; son substratum est constilué par des schistes cristallins, gneiss, miraschisles, pne traversent en tout sens les épanchements de granite, de rimulite of autres roches éruptives. C'est autom de cel îlol que le sol gaulois s'est peu à peu constitué par les députs successils des mers géologíques et par les mourements orogénirques, qui onl si sourent troublé la disposilion primilive le ses conches.

Emerge dess sa formation, les mers Cimbriemes ou Siluriennes n'ont pénétré que sur ses bords, saul dans la Montagne Noir oì elles out laissé de vastes dépôls.

Les mers déroniennes et carbonilères ont envalui an Nord les dépressions dı Benujolais et du Bombonnais, landis qu'au Sud elles recourraient la Monlagne Noire.

C'est alor's que, des l'époque honillère inférienre commence i s'effectuer un des mourements des plus prodigieux qui aient effecté l'ècorce terrestre en Europe, mourement qui a donnẻ naissance à la chan̂ne liereynienne.

Cetle chaine traversai! la France en décrirant une sorte de $\mathrm{V}$, dont le sommet se tronvait m pen an Sud du Massif Central. Cette région dtait alor's relién d'mne part à la Bretagne et à l'Angleterre, d'autre part aux Vosgges el à la Forêt Noire; ce relief s'est accentué pendant toute la durée de l'époque

(1) Boule M. Le Massif Central de la France.- Exir. du Dict. géolog. de la lirance par F. Joanne 1895. 
carhonifere pour atteindre son maximum a'd stephanien formant ainsi des montagnes élevées, convertes d'une riche vescetation.

Les agents almosphéripues effecherent leur auve de destruction, des phuies torrentirlles lésagrégirent les sommets des torrents aux crues violentes entrainerent daus les dépressions les muleriaux arraches a la monlagne, tandis que sur les hords du Massil, l'an charriait aree les graviers et les sahles, les détritus de la riche vigutalion de celte époque. Elle les entassait en couches nombreuses el domait ainsi naissance aux hassins honillers exploites anjourd'hui, tels que ceux d'Aulun, de brassac, Alais, Cilnampagnac, Commenty, Sainl-Elienne, ele.

Des phénomenes exruptifs imlenses plisserent et dislorperent les conches de houille. De ces anciens volcans, détruils par l'urosion datent des produits volenninues nombreux qui nous en révelent anjond'hui lexistense: Orlhophyre, porphyre quartzilère, porphyrites el mélaphyres, rendres el relinites.

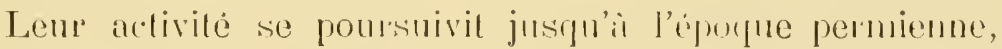
Landis que la mer envaluissail les défressions nouvelles du Massif Central, domant nilissance aux schistes litumineux d'Aulun, aux sehistes et grès rouges de Brives, Rodez, Saint-Allipge et Lodere.

Ere Secondairc. - A l'aurore de cette époque le Massil Central n'est plus qu'un fragment de la chaine hercynienne entouré de tous côtés par les eaux. De la France actuelle les mers jurassiques ne laissaient à décourert que le Massil Central, les Vosgres et la bretagne, séparés par les détroits du Poilou el de la Gite-d'Or.

Vers la fin du crélacé $u$ mourement général d'exhaussement ferme les deux délroils ef ratlache délinitirement le Massil Central aux conlrées roisines, constituant ainsi le squelette de la I'rance fulure.

Durant celte époque on ne conslate aucun phénomène 
d'éruption : c'est une ère de repos entre les émissions des roches anciennes et celles des roches ignées modernes.

Ere Trrtiaire. - Les temps tertiaires tronverent la surface dı Massil fortement dénudé, sans chaines de montagnes ni volcans, mais découpce par de larges curettes ou l'eau pourail s'accumuler.

"Une premiere série de lacs est jalonnée par les déprits " d'argile el de ealcaire lacustre du Bomrbonnais, de la " Limagne et du Cuntal. Une seconde série suivait la dépres" sion comprise aujourd'hni entre les chaines du Forez et " clu Velay d'une parl, el les monts du Beaujolais, du Lyon" nais et du Vivarais d'aulre part. Elle correspond anx "bassins de Roanne, de Montluison el du Puy; on trouve " dams les dépôts de ces lacs les déponilles des èlres virants " qui animaient les paysages de ces temps reculés. ") (1)

A l'éporfue miocine, un exhanssement lent du sol eut lieu; la mer nummulitique śéloigna et la plaine de la Limagne resta seul occupée par un immense lac d'eau donce yui s'étendail sans discontinuité de Moulins jusqu'à Asprières (Aveyron).

De larges lleuves roulaient lem's eamx dans un pays plat; c'est avant le déclin de celle période, alor's que les lacs se comblaient, que se produisirent de nouveaux ellorts orogéniques altestés par des hasaltes dont il ne reste aujourd'hui que des lambeaux épars.

$\Lambda$ près une période d'accalmie, l'activité éruptive reprend avec violence dés l'anrore du pliocène; ces elforts débutent arec le grand soulèvement des $\Lambda$ pes dont les plissements se poursuivirent dans cette régrion, comme l'a démontrẻ M. Michel Lévy.

Alor's se dressèrent l'imposant cratère du Canlal et les deux volcans accouplés du Mont-Dore. De celte éporne datent également les volcans dominitiques a cralicre de la

(1) Boule : liassif Central. 
chaine des Pıys el les massils du Mezenc et du Mégal qui duminent la vallée dı Rlione.

Ces reliefs volcaniques surajoutes an substratum de lintipue Massif Central en chandrent totalement la topographie. Il vinl s'y aljoindre encore it la lin du pliocene et du quaternaire : les chaines émplives du Deves, do l'Aulsac, et la remarquable guirlante des soixante volcans a cratère de la chaine des Puys rui se dresse au-dessus du bassin de la Limagne (Glangeaud).

Le Massil Central subit aussi une puissante période glaciaire. De rastes champs de névés s'établirent sur ses sommets; les roches moulonnées, striées, les accumulations monainicues, les bloes erratiques en sont les témoins. Puis un changement dans la température amena la fonte des glaces; c'est alors que des phénomènes d'errosion torrentielle crenserent les valleses actuelles. Une recrudescence de froid permit à de nonveanx glaciers de s'installer sur les cimes, de combler les vallées et d'achever le modele topographique alor's que l'homme prenait possession du sol.

Les sources thermales, les émissions de bitume, les dézagements d'acide carbonique sont les manifestations ultimes des éruplions anciennes.

\section{$2^{\circ}$ OROGRAPHIE}

Comme le dil avec heaucoup de raison M. Bonle, l'orographic dı Massif Central ne peutètre scientifiquement décrite qu'en prenant pour base les données géologinnes.

Les caractères physiques d'un pays sonl en eflet très étroitement liés a sa nature et à l'histoire de son passé.

Pour ce chapitre nous avons fails de nolables emprunts au magistral article publiẻ par M. Boule dans l'étude précédemment citée.

Les reliefs paléozoüques. - Si l'on fait abstration des régions volcaniques qui sont venus lardivement se superposer 
au relief ancien du Massif Central, on peut considérer ce dernier comme un plan incliné. Son bord relevée, formé par le système des Cérennes (1.500 mètres d'altitude) domine brusquement la vallée du Rhône tandis que sa partie occidentale, s'étend sur une série de plateaux (Limousin, Segalas) plus ou moins accidentés. Il s'abaisse ensuite graduellement vers le $\mathrm{N}$. et l'O. par une pente très faible le raccordant insensiblement aux plaines de la Loire et de la Garonne.

Le système des Cévennes part du Col de Naurouze et va jusqu'au Col de Longpendu (500 kilomètres) ; abstraction faite du Mézenc d'origine volcanique, ce sont les montagnes de la Lozère qui constituent la région la plus élevée de la chaîne dont le point culminant est à 1.702 mètres d'altitude).

Elles débutent au Sud par le Massif de la Montagne Noire, l'Espinouse et les monts de Lacaune, creusées par les vallées de l'Orle et de l'Agoût.

Les points culminants sont le Roc de Montalet (1.260 mètres) et le Pic de Marc (1.210 mètres) ; on peul hui rattacher le Levezou, dans l'Aveyron, et le territoire connu sous le nom de Segalas, à l'altitude moyenne de 700 à 1.000 mètres et dont le point culminant est le Signal du Pol, à 1.157 mètres. Les Cévennes se contourment par le Massif de l'Aigoual, dont le point le plus élevé est le Signal de l'Hort-Dieu (1.567 mètres). Au Nord ce sont les masses du Mont-Lozère, avec une cìme de 1.702 mètres, le Finiels.

La ligne de faite se dirige ensuite au $\mathrm{N}$. vers les montagnes du Goulet et la plaine de Montbel. Dans cette dernière région, à une altitude moyenne de 1.200 mètres, viennent se souder une suite de reliefs qui traversent le Massif du S.-E. au N.-O. par la Margeride (Signal de Randon, 1.554 mètres) et se continuent par le Cezallier.

En prolongation du Cezallier, les hauteurs d'Ussel relient les Cévennes aux reliefs limousins ; elles compremnent le plateau de Millevaches (Mont-Besson, 978 mètres) arec ses contreforts; la chaine des Monedières au Sud, les Monts 
Gargans i l'Ouest, le Bas Limousin, les monts de la Marche, au N.-R. les collines de Combrailles.

De la plaine de Montbel les Cérennes se continuent par la forêt de Banzon (1.510 mètres); elles envoient vers le Rhòne les crêtes du Tanargue et détachent ver's l'ouest les monts dı Velay ou clıaine du Devès, qui se poursuivent par le système du Forez.(Pierre sur-Haute, à 1.610 mètres).

Les Cévennes se prolongent par le soubassement du Mezenc el par la chaîne des Boutières (Mont Felletus, 1.890 mètres). lilles s'achèvent par des reliefs d'environ 1.000 mètres: Monts du Lyonnais, du Beaujolais, du Charolais et en grande partie par ceux du Mâconnais.

Le groupe du Morvan termine au Nord le Massif Central.

Les grands plateaux calcaires. - La mer jurassique pénétrait dans la partie mériclionale du Massif Central par un délroit aujourd'hui constitué par' le Séranne, dont le RocBlanc (910 mètres) domine la région des Garrigues et du Languedoc.

Les lépôts de cette époque forment une série de plateaux d'une altitude de 1.000 mètres: Causse du Larzac, Causse Noire, Mejean, de Sauveter'e, de Mende, de Rodez.

Les grandes dépressions ou bassins tertiaires. - Elles compremment deux groupes qui correspondent : l'un au bassin de la Loire, l'autre au bassin de l'Allier.

Dans la région arrosée par la Loire ce sont d'amont en aval: le bassin du Puy et de l'Emblavés; la plaine tertiaire du Forez (Montbrison) et celle de Roanne.

Pour l'Allier' ce sont: les bassins de Brioude, d'Issoire, la raste curette de la Limagne et le lambeau tertiaire d'Ambert.

On y rattache les bassins du Cantal (Aurillac, Mauriac, St-Flour) et ceux de la Lozère (Malzieu, St-Alhan, Javols) qui élaient autrefois en relation avec ceux de la Limagne, d'Auvergne.

La jonction de ces deux grands bassins s'effectue au 
Nord des montagnes de la Madeleine, oi ils forment les plaines du Bourl,omnais.

Les reliefss volcanigues. - Ils penvent être répartis entre trois grands groupes qui sont:

a) Le district du Velay, comprenant: les massifs phonolitiques du Megal el du Mezenc; les volcans de la chaîne du Devès et les plateaux basaltiques qui se relient à ceux des Coirons.

b) Le district du Cantal, comprenant: le massif du Cantal proprement dit, dont le vaste cratère est couromné par toute une série de pics, Plomb-du-Cantal (1.85S mètres), Puy Mary (1.806 mètres), Chavaroche, arec, an centre, le cone phonolitique du Griou (1.69' mères), le massil basaltique de l'Aubrac; de vastes platcaux de même nature (Planèze, Cezallier).

c) Le district du Mont-Dore, comprenant: le groupe du Sancy (1.886 mètres) et celui de la Binne d'Ordanche; au Nord, le système de la chaîne des Puys ou Monts •Dômes.

\section{HYDROGRAPHIE}

Le nond hydrographique du massif se lrouve situé dans la vaste plaine de Montbel. Tandis que les Cévennes séparent le hassin océanique du bassin médilerranéen, la Margeride, le Cantal, le Cezallier, les Monts-Dore, le platean de Millevaches et les monts dı Limousin délimilent les hassins de la Loire et de la Garome. Quant aux monls du Morvan, ils appartiennent tant au bassin de la Loire qu'à celui de la Seine.

Tous les cours d'eau dı massif, de débit lıès rariable, suivant les saisons, se répartissent en quatre bassins :

Bassin du Rhone. - Le bord oriental du masil fournit au Rhône des petits alfluenls, les Grosnes pour l'Azergnes; le Gier qui descend du Pilat, l'Ardèche, le Gardon. De l'Aigoual, descendent le Vidourle et l'Hérault. 
Bassin de la Garonne. - A l'exception de l'Ariège, lous les grands alfhents de la rive droite de la Garonne appartiennent au Massif central. Le Tarn prend sa source dans les montagnes de la Lozère. L'Agoût et ses aflunents reçoivent les eanx du versant septentrional de la Montagne Noire, dı versant mérilional des monts de Lacaune el du plateau de Lespinouse. L'Areyron et son aflluent le Viaur draînent toute la région clu Segala. Le Lot prend sa sonrce dans les montagnes du Goulet; il sépare l'Aubrac des Causses de Séverac. La Truyère qu'il reçoit à Futraygues sépare l'Aubrac de la Margeride et dı Canlal.

La Dordogne prend naissance auprès du Puy-de-Sancy; le Cantal lui donne plusieurs afluents: la Rue, la Symène, le Mar's, l'Auze, la Maronne et surtout la Cère. Du plateau de Millevaches et des monts du Limousin, descendent la Corrèze el la Vezère puis l'Isle et la Dronne.

Bassin de la Loire. - La Loire a son origine an Gerbierdes-Joncs. Sur sa rive droite, elle reçoit le Lignon, le Furens qui descend du Pilat, le Sornin, l'Arconce venus des monts du Charollais, l'Arroux du Morvan. Sur sa rive ganche, elle est grossie par la Berne, l'Ance, le Lignon Forézien et surtont l'Allier. Cet important cours l'eau sépare la Margeride des monts du Velay, puis reçoit la Senonire et l'Allagnon tandis que la Dore lui apporte les eaux des monts du Forez et la Sioule, celles de la chaine des Puys.

Le Cher, la Creuse, la Gartempe et la Vieme apportent au grand fleure les eaux du plateau de Millevaches.

Bassin de la Seine. - Il reçoit les eaux de l'Yonne et de son aftuent la Cau (qui prend naissance dans les monts du Morvan.

Lurs. - Ils se tronvent dans les régions volcanicues. C'est le groupe du Mont-Dore qui en possède le plus grand nombre : Chauvet, Servières, la Godivelle, Pavin, Guéry, Chambon, Aydat. Le Gour de Tazanat, appartenant ì la chaine des Puys. 
Le groupe du Mezenc possède les lacs de Saint-Front et d'Issarlès; le lac du Bonchet appartient à la chaîne du Velay. L'Aubrac offre plusieurs petits laes dont le plus important est celui de Saint-Andéol.

Le groupe du Cantal en est à peu près dépourvu; seuls, les lacs de Madic el de la Crégul existent à sa périphérie.

Enfin, sur les lauts plateaux du Massif central existent un grand nombre de marais ei de tourbières.

\section{$4^{\circ}$ CLIMATOLOGIE}

" Le Massil central, traversé en son milieu par le $45^{\circ}$ lati" tude, derrait jouir dı plus agréable des climals si le relief " des terres n'intervenait pas dans les questions de météoro" logie. Un autre factenr, l'Océan, vient encore compliquer" " et troubler la question. Le Massif central est, en elfet, " sullisamment éloigné de la mer pour ne pas avoir tous les " avanlages du climat marilime; il ne l'est pas assez pour "que les vents liumides el cliauds de l'ouest ne lassent pas " senlir leur aclion. Cet ensemble de questions, déterminées " par la posilion géographique, le relief el l'influence des " mer's caractérise ce que les météorologistes appellent le " climal auvergnal ou climal dn Massif central. Tandis que " sur une carte a grande échelle, le Massil central parait " compris entre le $11^{\circ}$ el te $13^{\circ}$ degré isolhermique, sa tem"pérature moyenne ammelle est en réalité inl'érieure à ce " chillre et re dépasse pas 9 ou 10 degrés. L'Atlantique " exerce son intluence sur celle rógion de même manière " que pour la France entière. Tandis que les isolhères ou " isollermes d'été travel'sent le massif dans une direction " qui ne s'éloigne pas lrop de celle des parallèles les " isolbares on isolhermes d'hiver décrivent autour de lui " des courbes concenlriques parallèles aux côles et de " direction perpendiculaiı à la précédente, ce qui revient à " dire que, pendant l'hiver, les elllures tièdes de l'Océan 
" réchanffent tonte une r'egion qui serait hien plus froide si

" elle ocenpait une position plus continentale

"C'est vraiment le Massil central qui commande la distri-

"bulion des direrses aires méléorologípues de la France.

"D'abord, parce que ces montagnes élevées sont une

" harrière puissante qui arrete les vents humides de l'Océan,

" condense la rapenr d'ean, et, par suite, isole le bassin du

" Rhòne oir de telles actions ne se font phas sentir, mais,

"qui, subissant au conlraire, l'influence méditer'ranéenne,

" acquiert de ce fait el par suite d'autres circonstances, le

" climat thodanien. Ensuite, parce que les rents de diverses

" directions sont profondèment modifiés après lemr traversée

" des régions élevées du Massif. Celui ci le délarrasse en

" été de leur vipeur d'eau et les rend très froids en hiver. a Tandis que les rents d'ouest font surtout sentir leur " action dans la partie occidentale du Massif, les parties "méridionales plus rapprochées du Midi reçoivent aussi de " la pluie il la suite des rents Sud, de sorte que la saison " des pluies varie sensiblement suivant les points considérés.

"Dans l'Ouest elles sont particulierement lréquentes en " automne. Dans les montagnes et vers le Sud du Massil, le " nombre des jours de pluie a une répartition sensiblement " égale pour le printemps et porr l'automne. La carte de la " répartition des pluies dans le Massif Central (moyenne " annuelle) montre l'importance énorme des reliels dans cet " ordre de phénomènes. Les monts de la Lozère sont ceux " qui reçoivent annuellement la plus grande quantité d'eau " (1.800 millimètres). Le Forez et les montagnes de l'Aurel'"gne accusent de 1.000 à 1.500 millimetres. Ces chiffres " s'appliquent également aux monts lu Limonsin a canse de "leur proximité plus grande de la mer et malgré la faiblesse " relative de leur altitude. Les grandes plaines du Bourbon« nais et de l'Allier présentent au contraire une moyenne " annuelle aussi faible que celle des grands territoires plats " d!ı reste de la France (500 millimètres environ). Cette zone " de faible intensité pluviale traverse tout le Massif Central 
" du Nord au Sud et, par la Margeride el les Cansses, va

"rejoindre le bassin de la Garonne, une grande parlie de " précipitations atmosplıériques tombe sous forme solide. "Mais tandis que la neige ne fait que de courtes apparitions " dans les vallées ou les plaines basses, elle s'accumule sur "les monlagnes et les plateanx et y persiste pendant la plus " grande partie de l'année, d'octobre ì mai. "Après avoir ajonté que le climal du Massil Central est " soumis partout à des varialions très brusques, nous aurons " donné la plupart de ses caractères grénéraux. ") (Boule). 
II.

\section{LES ZONES DE VÉGÉTATION}

\section{FLORE FOSSILE}

Les plus anciens documents sur la végélation du Massif Central nous sont fournis par les riches dépôts de l'époque carbonilère.

Flore houillere. - Les régétaux qui composent celte flore appartiennent i deux groupes : celui des Cryptogames acrogènes el celui des Phanerogames rymnospermes; on n'y olsserve ni Monocolylédones ni Dicolylédones angiospermes.

Lus Lycopodinées formaient à cette époque de grands arbres de 30 a 40 mètres de hauleur : les Lepidodendron et les Sigillaria.

Les Calamites, Equisélacées analogntes à nos Prêles, mais hautes de 4 i 5 metres, vivaient avec des Amnularia.

Des Calamodendron, des Cordaites, des Conifires (WValchia et Dicranophyllum) des Asterophyllites caractérisent cetle végrélation.

Nous ne dirons rien de la végétation des temps secondaires, le Massif Central proprement dit ne possédant pas de niveaux de cet àge.

F\%ore tertiare. - Pendant l'ère tertiaire, les condilions physiques el hiologiques jusiqu'alor's remarquablement muiformes se sont dilférenciés au point de produire la variété qui caractérise l’ère moderne. La prépondérence appartient aux Palmiers et aux arbres a feuillage caduc dont le milieu de l'ère tertiaire sera l'apogée.

D’après M. P. Marty, c’est à l'Eocène inférieur qu'il faut rapporter la luxuriante flore des schistes de Menat représentée par 26 espèces parmi lesquelles prédominent les Cupulifères, les Columnifères et les Laurinèes.

La llore des Arkoses du Velay est représentee par des Palmiers du genre Phonix accompagnés des végétaux qui 
permeltent de placer ce gisement sur l'horizon de l'Eocène moyen.

Les nombreux lacs oligocenes du Massif Central nous ont conservé des vestiges de leur parure végétale.

D'après Marion, on trouve à Ronzon des chênes à leuilles coriaces, des Pistachiers, Myrica, Mimosa.

Les arkoses de la Limagne renferment des Palmiers, des Myrica, des Cinnamomum, des Proteacées à faciés australien.

Le plus grand des lacs gevaudanais, celui du Nalzieu possède des chênes, des aulnes, des platanes et des camphriers.

Ajoutons quau Puy de Mûr, MM. F. Heribaud et Girand ont reconnu la présence dans un dépót aquitanien à Helix Ramondi, d'un mélange de Diatomées d'ean douce avec des Diatomées marines, des Hydrobies et des Cyrènes.

D'après de Saporta, la tlore miocène d'Furope est d'une remarquable richesse et accuse une température égale, clémente durant l'hiver et pluvieuse durant l'èté.

Le gisement de Gergovie étudié par de Saporla et M. l'abbé Boulay a fourni de très nombreuses espèces: Palmier's, Myriacées, Légumineuses tropicales, Laurinées, Myrsinées, Hamamelidées, Plaqueminiers, Charme etc. qui indirguent un climat encore sec et chaud dont la température moyenne n'était pas inférieure à 20 degrès ; c'est l'apogée de la végétation du Massif Central.

Le relief de la région, modifié par la surrection des Alpes, établit des divisions profondes dans la répartition des végétaux. Les types des pays cliauds durent s'abriter dans la vallée du Rhóne; nous les retrouvons à Charay et à Rochesauve; landis que dans les régions montagneuses vivait toute une population nouvelle conservée par les gisements du Cantal et des environs du Puy : aulne, bouleau, chàtaignier, saule, charme, noisetier, ormeau, qui se sont conservés jusqu’à nos jours. 
Durant le pliocène, les groupes volcaniques du Cantal, du Mont-Dore et du Mezenc se courraient d'une abondante vergélation durant les phases de repos. Les restes de cette riche flore sont conservés dans les Cinériles. Ce sont des aulnes, des tilleuls, des juglandées maintenant confinés dans les Caucase, un Planera de la mème région, des érables, des ormes, des chìnes, des trembles, des sassafras, des bamhous, une vigne, des fougères, etc. tous types de la zone tempérée chaude.

Le Cantal est particulièrement riche en gisements de cette époque : La Mougudo, Niac, Saint-Vincent, Las Clausades, le Vaulmier, Capels.

Dans le Puy-de-Dôme on doit signaler les tufs ponceux de Varennes, les lignites de Boutaresse, les cinérites de Perrier, dans la Haute-Loire les argiles de Ceyssac.

Pendant les temps tertiaires les Diatomées ont joué un rôle important dans la formation des dépôts sédimentaires qui s'accumulaient au fond des mers, des estuaires et des lacs. Leurs gisements sont nombreux dans le Massif et certains sont d'une étendue considérable.

Arec l'ère quaternaire nous assistons à l'exode des végétaux, que le froid chasse du Massif Central. C'est la première période glaciaire, qui aurait anéanti en France le platane, le sassafras, le chène de Portugal, les derniers représentants des canneliers et des palmiers, tandis que la végétation sylvatique se cantonnait à la périphérie du Massif.

Pendant le creusement des vallées, la flore interglaciaire s'épanouit; elle est composée de nénuphar's, qui se développent sur les eaux des lacs, tandis que des forêts de pins, de chènes, de hêtres, avec des aulnes et des tilleuls, s'étagent sur les pentes. Le sapin apparaît en dernier lieu. C'est un climat plus humide, plus clıad, et plus égal que le climat actuel.

Une deuxième période glaciaire ramène dans les plaines les espèces établies danś les montagnes et fait disparaître du 
centre de la France le laurier, le figuier et l'arbre de Judée. Moins importante que la précédente, son action a été moins désastreuse; aussi certaines espèces ont pu se maintenir et attendre des conditions meillenres d'existence. L'élablissement des tourbières marque la dernière phase de celte période.

\section{FLORE AC'TUELLE}

La situation géographique du Massif Central, entre les plaines chaudes du Midi et les terres fertiles du Nord, la variété des terrains qui le constituent, les différences considérables de reliel (1.600 mètres) qu'on observe entre les régions les plus basses et les sommets les plus élevés, les nombreux cours d'eau qui descendent de ces montagnes dans les vallées et dans les plaines sont autant de faits qui ont doté le centre de la France d'une régétation aussi riche que variée.

Ce qu'il faut remarquer surtout, c'est que cette llore possède de nombreux types de toules les principales régions de la flore française; les dernier's représentants de l'époque glaciaire sont restés accrochés aux cimes dénudées et froides du Mont-Dore et du Cantal; des espèces des Alpes et des Pyrénées, dı Jura et des Vosges conslituent les robustes associalions alpines et subalpines des montagnes de l'Auver'gne et du Velay; la véfétation des plaines du nord de la France et de l'Europe centrale est représentée dans la zone sylvatique moyemne ; les côteaux ensoleillés donnent asile à un grand nombre d'espèces méridionales de la région circumméditerranéenne ; les lacs possèdent des espèces hygrophiles particulièrement intéressantes; enfin il doit aux rivages océanique et méditerranéen les intéressantes colonies maritimes qui s'épanonissent au voisinage des sources thermales; c'est dire que les trois grandes régions : méditerranéenne, sylratique et alpine auxquelles on peut ramener les limites de presque toutes les espèces de la flore française, sont parfaitement applicables au Massif Central.

L'extension méditerranéenne s'y trouve caractérisée par 
l'olivier, les chênes an feuillage persistant et un certain nombre de plantes herbacées. Tandis que les espèces arborescentes sont cantonnées sur le bord méridional des Cévennes; les herbes dépassent singulièrement cette limite et quelques-unes arrivent en plein cour du Massif cantalien, remontant les vallées chaudes de proche en proche et à la faveur de certains passages des Cévennes. C'est ainsi qu'on rencontre dans les vallées de l'Aveyron et dans les gorges du Tarn le pistachier terebinthe, un jasmin, un ciste, une campanule, un ophrys, apportenant essentieilement à la flore méditerranéenne. Le grenadier s'avance jusqu'à Capdenac sur le Lot.

Aı milieu du massif cantalien, à Carlat, le figuier, le fenouil, le pourprier et yuelques autres plantes réprésentent une colonie méridionale arrivèe à l'altitude de $\$ 10$ mètres, au contact même de la llore subalpine. Enfin, certaines espèces méditerranéennes pénétrent dans la Limagne el les plaines de la Loire. M. l'abjé Charbonnel vient de trouver cette extension à Massiac el noter son existence dans la majeure partie de la vallée d'Allagnon jusqu'au bassin de Neussargues. Néanmoins, celte znne ne joue qu'un rôle lout à lail accessoire dans la végètation du massif.

\section{Zone inférieure ou Région des vignes}

Dans celte région caractérisée par la culture de la vigne se rencontrent :

Thalictrum flarum L.

Ulex eropaeus L.

Trifolium subterraneum L.

'Trifolium elegans Savi.

Tetragonolobus siliquosus Roth.

Vicial lathyroüdes $\mathrm{L}$.
Lathyrus tuberosus $\mathbf{L}$.

Oinanthe listulosa L.

Lysimachia nummularia $\mathrm{L}$.

Limosella aquatica L.

Butomus umbellatus L.

Ornithogalum umbellatum L.

RÉGion silvatiQue ou des Forêts

Celte région s'èlève jusqu'à l'altitude de 1.500 mèlres qu'elle dépasse rarement; mais, si celte région est naturelle 
dans son ensemble, il ne faut pas perdre de rue que les forêts recevant du climat la raison de leur existence, la zone silvatique embrasse non seulement les espaces converts de bois touffus, mais aussi les surfaces dénudées, les rochers déconverts, les cultures, les paturages, en un mot, toutes les régions soumises aux mêmes conditions climatériques.

Elle peut être subdivisée en trois zones bien tranchées:

$1^{\circ}$ Zone inférieure de 212 à 700 mètres d'altitude.

$2^{\circ}$ - moyeme de 700 à 1.400

$3^{\circ}$ - supérieure ou subalpine de 1.400 à 1.600 mètres d'altitude.

\section{$2^{\circ}$ Zone silvatique inférieure ou du Giataignier}

Les extensions méditerranéennes pénètrent dans la zone silvatique inférieure dont les limites supérieures sont fixées par la culture du chataignier qui, dans son allure générale accompagne la courbe de niveau de 600 mètres. Fille pénètre parfois dans la zone silvatique moyenne suivant les accidents dı sol et l'exposition des vallées; sa parlie inférieure présente de même des enfoncements profonds. Le Morvan, le Forez, le Limousin, surtout dans sa parlie méridionale, les bords de la vallée du Lot, l'Aubrac, le Segala, les gorges septentrionales de la montagne de Lacaune, le rersant oriental de foutes les Cévennes jusqu'au Morvan forment au Massif central une couronne ininterrompue de forèts ou de bouquets de chalaigniers; une flore herbacée avec cent espèces environ est renfermée dans les mêmes limites.

Les espèces herbacées qui se trouvent dans cette zone sont :

Androxemum ollicinale All. Adenocarpus parvilolius 1). C. OEnanthe pimpinelloïles L. Antinhinum asarina $\mathrm{L}$. Adonis flammea Jacq. Alyssum campestre L.
Asplenium lanceolatum Sinitl. Acnanthe fistulosa L. Androsace maxima L. Antriplex rosea L. Allium flarum $\mathbf{L}$. Alopecurus arundinaceus Poir. 
Acer inonspessulanmm L.

Astragalus monspessulanus L.

Armeniaca rulgaris lam.

Amggralus communis $\mathrm{L}$.

Asperula galioünles bieb.

Aster amellus I.

Ajuga chamateplis Selıreb.

Aristulochia clematilis $\mathbf{L}$.

Allium baniculalum L.

Allimm oleraceum $\mathrm{I}$.

Allimm vinale $\mathrm{l}$.

Mllum sphurrocephalum I.

Moprecurus agrestis 1.

degilops triuncialis $I_{\text {. }}$

Asplenium Ilalleri I). (.

Arliantum Caprillus Vemeris I.

Buplenrma juncenm L.

brizal minor I.

Brachypodium destachyon Riom el Silı.

Bulomus umbellatus I.

Bromus jalulus M. el li.

Bupleurum aristalmm G. G.

Bupleurum rotumblolium L.

Diromus Siquarrosus I.

bromus maximus besl.

Cistus salvifolius I.

Coronilla Enerus L.

Colnus mas I.

Centranthus calcitraja Duli'.

Camlums vivalrensis Jol'd.

Corduncellus milissimus b. C.

Campanma Erimus I.

Chlora perfoliata I.

Calaminta Nepela Clair.

Ceralucephalus falcalus Pers.

Cancalis leptopliglla L.

Centaurea sotstitialis L.

Campanula l'apunculoides I.
Convolvulus lineatus L.

Cyperus lomgus I.

Carex hordeistichos Vill.

Carex maxima siop.

Coronilla minima L.

Coronilla scorpiojes linch.

Cembarea fectinala $\mathrm{L}$.

Cenbaurea maculosil lam.

Chomdrilla jumcan L.

Crepis pulelua $\mathrm{L}$.

Comvolvulus cambalirical.

Chenoporlium Bobrys L.

Clemopodimm opmlilolimm Schad.

Chenoporlimm vulvaria $\mathrm{I}$.

Clenopquilium intermedium Mert. el k.

Castanea vulgaris lamk.

Cephalantheras rulıa $\mathrm{N}$.

Cephalantheras ensifolia Sir.

Carex distans I.

Carex tomentosa I.

Carex Schreberi IVild.

Calex bisticla lluds.

Carex Ilalleriana Asso.

Dalura Stramonimu L.

1)iplolaxis mulalis 1). C.

Erucastrum oblusangulum lichb.

Elodium ciconimm Will.

Echinops sphurocephalus $\mathbf{L}$.

Erica vagans $L$.

Eragrostis minor $\mathrm{l}$.

Ełpuselum variegratum Schleich.

Emplubloia exigua I.

Euphorbia l'alcala l.

Epipaclis microplyylla Sw.

Gentiana ciliala l.

Glolublaria vulgarris L.

Gaucium comiculatum Curt.

Grammilis leptopluylla Siv. 
Genista germanica L.

Gratiola officinalis $\mathbf{L}$.

Gladiolus illyricus Kioch.

Gladiolus segelum Grawl.

Jelianthemum salicifolium Pers.

Helminthia echiö̈des Gartn.

Hollonia palustris J.

Hutchinsia petroa Brown.

IJjdrocharis Morsus-Rana $\mathrm{I}_{\text {. }}$.

Inula graveolens Desf.

Inula squarrosa L.

Inula bifroms L.

Inula salicina L.

Inula montana $\mathrm{L}$.

Inula britannica L.

Iberis amara $\mathrm{L}_{\text {. }}$.

Keutroplyyllum lanatum Duby.

Kícleria Selacea Pers.

Lychnis coronaria Lam.

Linum gallicum $\mathbf{L}$.

Linum strictum IIuds.

Lotus augustissimus I.

Lathyrus neglectus Puel.

Lytlıum hyssopifolia I.

Leucantlemum cebennense D. C.

Lobelia urens L.

Limodorum abortivum Sw.

Linum limanense Lamotle.

Linum tenuilolium L.

L.upinus reticulatus Desv.

Limạria supina Jesl.

Lindernia pyxilaria AJl.

Lemna arrhiza L.

Limum augustifolium Huds.

Jotus tenuifolius Rehb.

Lathyrus Nissolia L.

Lathyrus sphaericus Retz.

Lathyrus tuberosus L.

Lonicera ctrusca Santi.
Lactuca viminea Link.

Lacluca perennis L.

Linaria spuria Mill.

Linaria Elatine Mill.

Jinaria cymbalaria Mill.

Linaria Pelliceriana D. C.

Lolium rigidum Gaud.

Myagrum perfoliatum L.

Medicago ambigua Tord.

Melilotus parvillora Desf.

Marsilia quadrifoliata L.

Medicago falcata L.

Micropus erectus L.

Myosotis Lappula Moench.

Muscari racemosum D. C.

Mıscari comosum Mill.

Nardurus unilateralis Boiss.

Oxalis corniculata $\mathrm{L}$.

Ononis natrix $\mathrm{L}$.

Orobanche cruenta Bert.

Orobanche Picridis Schultz.

Odontites lutea Rehb.

Ophrys Scolopax Car.

Ophrys pseudo-bpeculum D. C.

Orchis pyramidalis L.

Osmunda regalis $L_{\text {. }}$.

Ophrys muscilera IIuds.

Ononis columno: All.

Onobrychis supina I). C.

Orlaya grandillora Ilolfm.

Orchis hircina Crant\%.

Orchis purpurea Huds.

Orchis laxillora Lam.

Polygala calearea Scluultz.

Phalangium ramosum lam.

Papaver hybridum L.

Potentilla supina $\mathrm{L}$.

Potentilla micrantha Ram.

Peltoselium segetum lioch. 
Plantago carinata Schrad.

Polygonum Bellardi All.

Phleum asperum Jacy.

Polypogon monspeliense IJesl.

Poa dura Scop.

Pilublaria globulifera L.

Prunus Nalaaleb L.

Persica vulgaris Miller.

Pencelanum alsaticum L.

Podospermun laciniatum D. C.

Physalis Mlkekengi L.

Plantago coronopus L.

Plalangium Liliago Schreb.

Plantago graminea Lam.

Ranunculus Lenomandi Schultz.

Ranunculus Sceleralus L.

Ruscus aculeatus $\mathrm{L}$.

Liapistrum rugosum . III,

Reseila Plıyteuma L.

liumex maritinus L.

Silene gallica $\mathrm{L}$.

Sedum anopetalum D. C.

Silshorpia europora L.

Salix incana Schr.

Silene conica $\mathrm{I}$.

Stipa pennata $\mathrm{L}$.

Seseli coloralum Ehrh.

Specularia hylurida H. C.

Samolus Valerandi $\mathrm{L}$.

Sculellaria hostifolia L.

Salvia Elliopis L.

Stachys herachba All.

Sisymbrium Irio L.
Scilla artumnalis $\mathbf{L}$.

Salvia Sclarea L.

Salvia Verbenaca $L$.

Stachys germanica L.

Spiranthes irstivalis Rich.

Trigonella monspeliaca $L$.

Trifolium maritimum II uds.

Tetragnnolobus silinuosus Roth.

Tillea muscosa L.

Trinia vulgaris I). C.

Teucrium montanum L.

Teucrium Scordium L.

Tulipa silvestris $\mathbf{L}$.

Tragus racemosus Desf.

Trifolium rubens L.

Trifolium scabrum L.

Trifolium glomeratum $\mathrm{L}$.

Turgenia latifolia Iloffn.

Tragopogon crocifolius L.

Thesium divaricatum Jan.

Valerianella coronata D. C.

Yerbaseum maiale J). C.

Vicia serratifolia Jacq.

Vicia villosa liobl.

Veronica prereor All.

Vitis vinileril $\mathrm{L}$.

licia gracilis Lois.

Veronica Tencrium $\mathrm{I}$.

Veronica tripliyllos $L$.

Kanthium macrocarpium D. C.

Xanthium strumarium L.

Zannichellia palustris $\mathbf{L}$.

$3^{\circ}$ Zone silvatigue noyenne oU DU GhÈne

Si nous pénétrons dans la zone silvatique moyenne c'est un autre sol, in autre climat. Sur les hauts plateaux de la Corrèze, sur les llanes des monts du Puy-de-Dòme, du 
Cantal, de l'Aubrac, de la Lozère et de Lacaune, le hêtre seul ou associé au chêne, qui l'accompagne jusqu'à 1.000 mètres, forme toutes lès forêts. Dans le Gevaudan, le Velay, le Mezenc, le Forez le pin silvestre joue un grand rôle au point de vue de la végétation arborescente; le sapin forme de belles forêts, au Mont-Dore, au Cantal et aux environs de Pierre-sur-Haute.

Ici comme dans le centre de l'Europe le hêtre est accompagné de Betula alba, Quercus pedunculata et sessiliftora, Ulmus montana, Fraxinus cxcelsior, Acer pseurlo platanus, Sorbus aria et aucuparia, Fagus silvatica, Abies pectinata, Larix europora, Pinus silvestris et uncinatus, Picea excelsa.

La flore herbacée de cette zone est excessivement riche en plantes robustes aux fleurs éclatantes et arbrisseaux élégants. En réalité la zone silvatique moyenne peut-èlre considérée comme le vrai centre d'oú partent toutes les espèces silvatiques; les unes descendent dans la zone inférieure et s'installent en colonies plus ou moins nombreuses dans les vallées fraiches et boisées, d'autres s'élèvent jusqu'au contact de la région alpine.

Nous citerons :

Arabis cebennensis D. C.

Asarum europoum L. I.

Anemone ranunculoides L.

Astrantia major I.

Andromeda polifolia L.

Atropa Belladonna J.

Cirsium rivulare Link.

Carlina cynara Pourr.

Cotoneaster vulgaris Lindl.

Circcea intermedia Ehrh.

Cicuta virosa L.

Cirsium Erisithales Scop.

Carluus personata Jaç.

Centaurea montana L.

Centaurea nigra L.
Crepis succisifolia.

Campanula latifolia Tausch.

Campanula linifolia Lan.

Calamintha grandillora Monch.

Crocus vernus All.

Carex filiformis $\mathrm{J}_{\text {. }}$

Carex paniculata I.

Carex teretiuscula Good.

Calamogrostis laceolata Roth.

Calamogrostis silvatica D. C.

Dentaria digitala Lam.

Dianthus granilicus .Jord.

Dentaria pinnata Lam.

Dianthus silvaticus Hoppe.

Doronicum austriacum Jacq . 
Dapline Laureola L.

Epilobium spicatum Lam.

Euphrasia minima Jacy.

Euphorbia lyyberma 1.

Eriophorum latifolimu Hoppe.

Eriophorum angustifolium Roth.

Eriopliorum vaginatum L.

Elymus enropuns L.

Eyuisetum silvalicum L.

Fritillaria Meleagris L.

Galium rotundifolium L.

Geranium lucidum L.

Geum rivale $\mathrm{L}$.

Galium boreale L.

IIesperis matromalis L.

Ilỵericum montanum L.

Iypochuris maculata $\mathrm{L}$.

Hieracium cerinthoides I.

Ilieracium prenantoïdes Vill.

Isoctes lacustris L.

Isoetes echinospora Dur.

Jasione perennis Lam.

Ligularia sibirica Cass.

Lonicera nigra $\mathrm{L}$.

Lysimachia nemorum L.

I.uzula nivea D. C.

Lycopodium clavalum L.

Lycopodium inundatum $\mathrm{L}$.

l.ycopodium chamacyparissus A.

Br.

Mentha cantalica IIerib.

Meconopsis cambrica Vigr.

Melampyrum silvaticum L.

Neotlia cordata Rich.
Orobus vernus $\mathrm{L}$.

Orjeoces palustris Pers.

Urchis montana Schm.

Orchis viridis Crantz.

'yrola chlorantla Swartz.

Pyrola unillora $\mathrm{L}$.

Petasites allus Giertn.

Pyrola rotundifolia L.

Pyrola secunda L.

Polemonium curuleum L.

Pulmonaria azurea Bess.

Pedicularis foliosa L.

Polypodium Pliagopteris L.

Polypodium Dryopteris L.

Polystichum spinulosum D. C.

Rhamnus alpinus L.

Rubus glandulosus Bell.

Rosa alpina L.

Ribes petrieum Wulf.

Ribes alpinum L.

Stellaria nemorum L.

Sedum anmum L.

Senecio Cacaliaster Lam.

Swertia perennis L.

Salix repens L.

Scilla Lilio-Hyacintlius L.

Schenchzeria palustris L.

Tulipa celsiana Vent.

Thalictrum aquilegifolium L.

Thlapsi virens Jard.

Thlapsi brachypetalum Jord.

Trifolium spadiceum L.

Thesium alpinum L.

Viola sudetica Willd.

1.' ZONE SILVATIQUE SUPÉRIEURE OU SUBALPINE

La zone silvatique supérieure débute à la limite des forêts, les sapins et surtout les hêtres devienuent buissonnants puis 
disparaissent vers 1550 à 1600 mètres; ils sont remplacés par des pîturages on des crêtes rocheuses.

En réalité celte bande qui ne dépasse pas 200 mètres en altitude, sourent désignée sous le nom de zone subalpine sert de transition entre la région des lorêts et la région alpine. Fille n'a sa raison d'ètre que dans ce fait, que certaines espèces ne trourent des conditions conformes à leur existence que sur la lisière supérieure des forêts oì elles sont localisées.

Les espèces subalpines s'élèvent parfois jusqu'à 1.700 mètres comme au Mont-Dore, d'autres descendent acciden-tellement à des altitudes infériemres à 1500 mètres.

Les espèces caractéristiques de cette zone sont :

Angelica pyrenca Spreng.

Bupleurum ranunculoides L.

Crepis lampsanoides Froël.

Carlina Nebrodensis Guss.

Crepis grandiflor: .

Epilobium origanifolium Lam.

Epilobium trigonum Schkrank.

Genisla purgans L.

Ilieracium cerinthoides L.

IIieracium aurantiacum L.

Juncus alpinus Vill.

Juncus filiformis L.

Koleria cristata v. restita Herib.

Lonicera alpigena $\mathrm{L}$.

Leontodon pyrenaicus Gouan.

Lycopodium Selago L.

Orchis globosa L.

Orchis nigra Scop.
Orchis albida Scop.

Potentilla aurea L.

Pinus uncinata Ram.

Pedicularis comosa L.

Poa sudetica Ilanck.

Ranunculus platanilolius L.

Rubus saxatilis L.

Silene Saxilroga L.

Sisymbrium pennatilidum D. C.

Silene rupestris L.

Senecio Fuchsii Gmel.

Swertia perennis L.

Salix lapponum L.

Salix phylicifolia L.

Selaginella spinulosa A. Br.

Thalictrum Delarbrei Lamotle.

Viola billora $\mathrm{L}$.

Vicia orobus D. C.

\section{$5^{\circ}$ KONE ALPINE}

La zone alpine débute ver's 1.600 mètres, l'exclusion des arbres lui donne une physionomie toute particulière et caractéristique; elle est formée par des paturages naturels où 
l'herle croit drue et serréc. Sur le Massil Central elle se délache en six îlols : L'Aigoual, le Mont-Lozère, le Mezenc, Pierre-smr-llaute, de très petit diamètre, le Mont-Dore et le Cantal oì lenrs plages très irrégulières à cause des vallées occripent un grande superficie Dans celte zone végète sur quelines points le saule de Lapons (Salix lapponum et herbacea) le genevrier nain (juniperus nana) formé dans des tonfles irrégulières entre lesquelles rampent plusieurs Lycopodées; mais le Massif Central manque de Rhododrendons qui font le plus bel ornement de la zone correspondante dans les Alpes et les Pyrénées.

Les espèces herbacées de celte zone sont :

Anemone rermalis $\mathrm{L}$.

Achillea pyrenaica Sibslı.

Allosorus crispus Bernh.

Asplenium virile lluds.

Asterocarpus sesamoides Gay.

Androsace carned $\mathrm{l}$.

ג juga pyramidalis $\mathrm{L}$.

Agrostis rupestris All.

Avena montana Will.

Avena versicolor W'ill.

Aspidium Lonchitis Sw.

Bartsia alpina $\mathrm{L}$.

Cochlearia pyrenaica I). C.

Cirdamine resedifolia L.

Carex vaginata Tausch.

Cerastium alpinum L.

Cirerra alpina L.

Draba aizoiıles L.

Dianthus cessius Smith.

Epilobium alpinum L.

Erigeron alpinus L.

Empetrum nigrum L.

Festuca pilosa Hall.

Festuca nigrescens Lam.

Genista prostata Lam.
Gnaphalium supinum L.

Geum montanum $\mathrm{L}$.

Galium anisophyllum.

(ialium boreale L.

Gentiana verna Vill.

Hieracium glanduliferum Hoppe.

Ilieracium piliferum Hoppe.

Jasione humilis Pers.

Luzula Desranxii Kunth.

L.uzula spicata D. C.

Luzula sudetica D. C.

Meum Mutellina Giertn.

Myosotis alpestris Schmilt.

Phytemma betonicifolium Vill.

Pedicularis verticillata $\mathrm{L}$.

Phyteuma hemisphoricum L.

Plantago alpina $\mathrm{I}$.

Phleum alpinum L.

Poa alpina $\mathrm{L}$.

Polypodium alpestre Hoppe.

Silene ciliata Pourr.

Sagina Linnaei Presl.

Saxifraga hieracifolia Waldst et liit.

Saxifraga oppositifolia L. 
Saxifraga androsacea $\mathrm{L}$.

Senecio pyrenaicus Cr. Cr.

Salix herbacea L.

Sorbus Chamamespilus Crant\%.

Sedum alpestre Vill.

Saxifraga loryoides L.

Saxifraga exarata Vill.

Senecio Doronicum L.

Soltlanella alpina $\mathrm{I}$.
Strep,topus amplexifolius D. C.

Trifolium badium Sclireb.

Trifolium glareosum Schleich.

Tozzia alpina $L$.

Veronica saxatilis Jacy.

Veronica urticifolia L.

Vaccinum Vilis-idwa L.

Veronica alpina $L$.

\section{$6^{\circ}$ Flore lacustre}

Le trait le plus saillant qui caractérise la llore des lacs est la régularité avec laquelle les plantes sont réparties à leur surface ou dans leur profondeur. Presque toujours on les observe groupées en zones distincles allant du bord au centre, c'est ainsi que se jrésentent successivement:

$1^{\circ}$ Une zone littorale de plantes déversées lor's de l'eau: Pluragmites, Scirpus.

2. Une zone inférieure de plantes à feuilles nageantes dont la plus commune est le Nuphar luteum.

$3^{\circ}$ Une zone concentrique de plantes à tiges fenillées arrivant à la surface de l'eau ou à son voisinage, surtout constituée par' des I'otamorgeton.

$4^{0}$ Enfin une surface nue, mais d'où le grappin ramène des plantes de fond: Chara, Nitclla, elc.

Certaines plantes normalement llottantes peurent être considérées comme formant une llore pélagique macrophytique.

A côté de ces végrétaux supérieurs d'une détermination relativement aisée, se trouvent les $\Lambda$ lgues dont l'étude longue et pénible est généralement délaissée. Une seule famille, celle des Dialomées, a été étudiée en détail par F. Hérilsaud.

Dès 1900, M. Bruyant a fait connaître le résultat de ses recherches sur la plankton des lacs du Massil Central. Les pêches au filet fin elfecluées au Chauvet et au Pavin ont démontré l'existence d'une infinité d'êtres vivants en suspension dans l'eau et dont l'ensemble constitue le Plankton 
pélagique. Ce dernier comprend ì la fois des végélanx el des animanx; des $\Lambda$ gaves d'une part et del'aulre des Protozoaires, les Rotilères, des Entomostracées.

\section{$7^{\circ}$ Flore des tourbieres}

Les tombières présentent un aspect triste et monotone qui lenr a ralu en Aurergne le nom de Les Mortes. Eilles sont très nombreuses dans le Massif Central oì elles occupent un grand nombre de curetles de toutes dimensions. Ces gisements ne présentent en général qu'une faible épaisseur, mais l'on ne peut rien présumer à l'égard des plus importants, qui n’ont jamais été exploités à fond.

Les tombières sont nombreuses dans la zone inférienre des plaines, lien que les travaux d'assainissement et de culture en ail beanconp réduit l'ètendue et l'importance.

Elles portent une régétation très uniforme (Spliciognes,

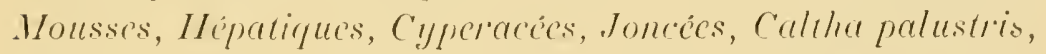
Drosera, Comarum palustre, Vulcnaria dioica, Menyanthes, Wrihlenbergir, Pedicularius, etc. à laquelle viennent s'ajouter dans les zones supérieures quelques especes des liauts sommets (Andromeda polifolia, Taccinium uluginosum, Eriophorum alpinum, E. vaginatum, Allium victoriale, etc.). L'aulne, le bouleau, des saules accompagnent généralement celle flore herbacce. Souvent, des arbres isolés on en massifs s'y trourent ensevelis, par exemple à Jassy et à Boutaresse, sur les monts du Luguet. Certaines tonrbières, comme celles des Cajasses et des Vacants, à la Godivelle, paraissent aroir été formées par des arbres et des végétaux transportés là par quelques grandes inondations.

\section{Sn Florule halophile}

Les sonrces minérales de Saint-Nectaire, Sainte-Marguerite, Vic-le-Comte, etc. répandent des eaux chargées de chlorure de sodium. Les terrains qu'elles arrosent portent une florule halophile bien caractérisée par: Trifolium marit., Taraxa- 
cum leptocephalum, Carex marit., Plantago graminea var. marit., Triylochin marit., Glyceria distans, Cleara crinita var. brevispina, elc.

\section{$9^{\circ}$ Florule boréale}

La flore de l'époque glaciaire a légué à la flore actuelle du Massif central un certain nombre d'espèces qui vivent sur les hauts sommets hattus par les vents du Cantal et du Mont-Dore. Ce sont : Gnaphalium norecegicum, Salis. Iapponum, Woodzia hyperborea, Carex raginata, Jasione humilis, enfin le Saxifiaga hieracifolia, qui habite les corniches basaltiques du Pas-de-Roland; parmi les mousses: Zygoda laponicus, Dryum arcticum, Trimmia norveyica, elc. Il est curieux de noter les analogies remarquables qui existent entre la flore des montagnes d'Auvergne et la flore des Vosges; la liste des espèces communes aux deux régions est très longue, ce qui semblerait indiqner que les populations régétales actuelles de l'une et de l'antre ont une origine commune : les $\Lambda$ lpes, les Pyrénées et les contrées borẻales. Mais l'observation la plus importante à laire est l'inlluence de l'altilude sur les caractères spéciliques des végétaux. Le F. Héribaud a fait olsserver en 1891 que pour une mème espèce de Diatomées virantes à deux allitules très différentes, comme les bords du Lot et le sommet du Plomb pour le Cantal, les élangrs de Lezoux et le pic de Sancy pour le Puyde-Dóme, la for'me des hautes altitudes, ses valves siliceuses munies de slries plus nombreuses et moins lortes que la forme provenant de la zone inlérieure.

Ce fait élant bien établi pour les Algues microscopípues, on peut se demander si les autres groupes végétaux offrent des variations nolables en s'élevant de la zone inférieure sylvatique aux altitudes superieures; on sait qu'à une altitude différente correspondent une température et une hygroscopicite de l'air différentes, ainsi qu'un éclairement plus intense; il est donc rraisemblable, à priori, qu'une mème espèce, suirant qu'elle sera exposée à des conditions climatẻriques 
différentes, sa physionomie s'éloignera plus ou moins de la lor'me typique, tout en conservant les caracteres intrinsèques de l'espèce; il résulte, en eflet, des belles expériences faites nar M. Bonnier, membre de l'Institut, fu'me mime plante cultive en plaine et it haute altitude, acquiert dans ce dernier cas des dillérences plus on moins profondes tant au point de vue des caractères morphologirues que sous le rapport de la structure interne.

Ainsi la tige reste plus courte et rampe souvent sur le sol, le limbe foliaire devient plus épais el plus riche en chlorophile, la fleur est plus grande et d'une coloration plus vire. Pentant la belle saison les racines opèrent un emmagasinement de réserves nutritives plus abondantes, réserves qui seront utilisées par la plante pendant le long hiver qu'elle aura à traverser.

Les espèces alpines n'ont guère que qualre à cinq mois pour accomplir les plases de leur évolution; ainsi, à peine la neige est elle fondue que la plante restée stationnaire pendant sept à huit mois, se hate de dérouler ses fenilles, d'épanouir des lleur's el de mirrir ses fruils. Il en résulte que certaines especes annuelles dans la plaine devienmment viraces aux hautes altilndes. Ce fail doil ètre altribue selon nous an développement exagéré quacquiert le sysleme souterrain, sous l'influence du repos prolongé anquel la plante est condammée pendant les sept à huit mois qu'elle reste sous la neige. Le phénoméne inverse, c'est-ì-dire la descente dans la plaine des plantes des altitudes élevées, se produit aussi, mais beancomp phus rarement, les hotanistes herlorisant savent, en ellet, que les espèces de la finine ont plus de tendances à remonter les cour's d'eau et les vallées, que les plantes alpines n'en manilestent à s̈installer aux basses altitudes. 


\section{BIBLIOGRAPHIE}

\section{TRAYAUX CONSULTES POLT CETTE E'TLOE}

Aliovist. - Botanik, 1898 (Norstedts fürlag).

A Lagvist och LAGeistedt. - Läroboki Nalurkunnighet (Norstedts Fürlag).

Anderson. - Svenska Växtvärldens Historia (Norstedts fürlağ).

Arescioug. - Bolanikens Elementer (Gleerups förlas).

Arescrorg Eril. - Plantie cotyledonie Florie Gothoburgensis

(Londini Gothorum 1836).

Aresciotg F. W. - Shines flora. 2. uppl. Lund 1881:

Arnell. - Vegetationens utreckling i Sverige. Upsala 1878.

Ascuenton, P. I. - Potamogetonaceo, Hydrocharitacece, Engler Natürl, I'flanzenfam. II Theil, Abteil. I.

Ascienton, ?'. I. - Die geographische Verbreitung der Seegräser, Petermann's. Mittheil. 1870.

Auvergne, enty et fontits. - Ann. 1673-76. Grand in-fol. Plans des forêts sur velin. Ms. Bibl. Clermont-Fd.

BArBer EDwARD. - Through Auvergne on foot. Griffeth and Farrar, London, in - $8^{\circ}$.

Beille Lucien. - Essai sur les zones de végétation du Massif central de la France. Broch. in $8^{\circ}$, Toulouse.

Beille Lucies. - Limite altudinale du châtaignier sur les pentes ouest et sud ouest du Massif central de la France (Assoc. franc. avanc. des sciences) $16^{\circ}$ sess., Toulouse.

Bergigen S. - Bidrag till liinnedom om Fanerogamfloran vid Discobugten pá Grönlands Vestkust. (Kgl. Vet. Ak. Förlı, 1871. No 7. Sthlm).

Bergstiand. - Geologi 1868 (Arrheniu's förlag).

Berlin Ári, - Kïrlväxter, insamlade under den svenslia expedi. tionen till Grönland 1883. (Öfversigt af Kongl. Vetenskaps Akademiens Förhandlingar 188. n 7 , Stockhohm).

Bertiold G. - Ueber die Vertheilung der Algen im Giolfe von Neapel nebst einem Verzeichniss der bisher daselbst beobachtigten Arten. Mittheilungen a. d. zoolog. Station zu Neapel. Bd. III 1882. 
Brotmino, - Reboisement de l'Auvergne (Bull. de la soc. d'Ilort. el de Vit. du l'uy-(le-Dôme).

Bu,re Axk. - Forsog till en 'Theori om Indvandringen of Norges Flora under vexlende regnfulde og lorre Tider (Nyt Mag. for Natury. 2 I de Bind), Christiania 1876.

Bı, M. N. - Norges llora : Flora Norveg., 3 bd. m. suppl. Gr. in 8". Christiania, $1861-76$.

Bıутт M. N. - On vegetalionsforholelene ved Sognefjorden. Christiania 1869.

Bu.MrT M. N. - Essay on the immigration of the norregian flore. Eligler botanis. Jahrb. Syst. II p. I (roir (i. J., X.142).

Bors.s, Аввк. - Flore pliocène du Mont Dore avec 10 pl. lit.l et nombreuses figr. append. et notes diverses. Gr. in-1". Paris, Sayy.

Borlal; Arus. - Notice sur la flore tertiaire des environs de Pri. vas. Bull. Soc. Bot. de France; 1 partie IXXYIX, page 227. 2. partie IXXXIX, p. 225.

Bolle, M. - Les volcans de la France centrale (Vojez VIIIe Congrès, 9 col. intern. 1900). - Guide gréolog. en France, lixcursions en France. Lille, Le IBigot frères, in 8 ".

Boxitir. - Les plantes arctiques comparées aux mêmes espèces des Alpes et des l'yrénées. - Revue générale de Botanique. Tome VI, 189 '.

Boxiser. - Culture expérimentale daus les Alpes et les l'yrénées. Revue gén. de Botanique, T. II.

Boxiner. - Elude expérimentale sur l'influence du climat alpin sur les végétaux etlesfonctions des plantes (Bull. Soe. Bot. de France).

Boxwer. - Cultures expérimentale dans les hautes altitudes (Comp. rend. au Soc.)

Boxier. - Recherches expérimentale sur l'adaptation des plantes au climat alpin. ( Inn. des Sciences Nat. T"ser. F. XX).

Boxiser G. et Flanarer Cir. - Observations sur les modifieations des végétaux suivant les conditions plıysiques du milieu. (Ann. des Sc. nat. G" ser. J. V'II 1879).

B3.MEn A. - Programme de la flore du centre de la France suivi d'un calalogue des plantes observées dans le rayon de cette tlore, in $8^{\circ}$ Nevers.

Batear A. - Flore du centre de la France el du bassin de la Loire (3" édit. in $\left.16^{\circ}\right)$.

Buci'on J. P.- Histoire naturelle de la ci-devant province d'Auver- 
gne, extr. de la grande collection d'histoire nat. de J. P. Buch'oz, Paris, in- $f^{\circ}, \mathrm{pl}$.

Candolle, (de). - Rapport sur un voyage botanique et agronomique dans les départements du centre. (Mén. publ. par la Soc. d’agr. de la sitine F. IV 1812.

Cingolle, (de). - Rapp. sur deux voy. lot. et agron. dans les dép. du nord et du centre (1'aris in $8^{\circ} 120$ p. 1813).

Cinrist I1. - Das P'llanzenleben der Schweiz, Zürich 1879.

Curve A. - Zum Pllanzenleben in Nordechwedischen Hocligebirgen Stockh. $1901.8 \mathrm{~m} .5$ lipfrt.)

Curtel, ( l'assimillation pendant les nuits norvégiennes. (Revue gén. de Bot. Tome II, 1890).

DAmlstedt. H. - Hieracia Suecica. Bidrag till sydüstra Sveriges llieraciumflora. (9 Th. Stock. 18909 ' gr. in-4).

DEL MiBre. - Flore de la liaute et basse Auvergne, ou recueil des plantes observées sur les montagnes du P'uy.de-Dóme, du MontDore el du Cantal. (2) édit. 2 lom. en 1 vol. Clermont-Fd.) 1800.

Donel. Port. A. - Farbenpracht und Gï̈sse der Alpenblumen. (Kiosmos Bd. 1879).

Düren Magxus IV. von. - Conspectus regetationis Scania (Lundiic).

Dumas-Danond. - Contrib. a la flore bryolog. de l'Auvergne (Rev. Scien. du Bourb. et centr. de la France p. p. 107, 152).

Dúśn K. F. - Om Splagnaceernas uthredning i Sliandinavien. (Beitr. 1887. gr. 4. m. color. Kart in fol.)

EuLunu. - Bidrag till Kännedomen om Sveriges Klimat. Kigl. Vetenskapsak. Ilandlingar.

Evielmany, Tu. IV. - Farbe und Assimil. (Bot. Zeitung 1883).

ERumix. - Geologi, 187' (Norstedts förlag).

FAtB. - Stormar orh Jordbüfningar, 1887 (Adolf Bonniers fürlag).

Forssel. - Botanik, 1902 (Beijers fürlag).

Fonstst. - Inledning till Botaniken (Beijers fürlag).

Forssell. - Kortfattad Lïrobol Botanik (Beijers fürlag).

Flanicte. - Le climat de la Scandinavie dans ses rapp. avec la végét. (Rev. des quest. scient. 1880 et ann. des Scien. nat. 6 ser. Tome IX p. 159, avec 2 cartes).

FrıEs. - Lïroboli i Systematisli Botanik, 1897 (Beijers fürlag).

Fues E. - Lichenographia Scandin. 2 part. Upsal, 1871-74, 80. 
Fracs E. - Summa regetatibum Sicandinavio, a part. Holm. 181649.80 .

Fuas lí - Botanislia utflykter. (Saml. al strödda tillfïllighets-

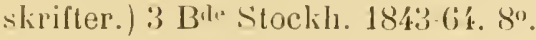

Fries k. - Idem opus. 2 rol. I ond. Goth. 18:8-4:2. 8 IIf $\%$

Frites, Elass. - Ilelsinglands cotyledonea och Heteronemea ((ietle, A.-1'. Landin, 185'4).

limes, Euns. - Observationes criticie l'lantas suecicas illustrantes (Upsalia).

Gasilaex Fre. - Contrib. a la flore des lichens du Plateau Central (Actes de la Soc. Liün. de Bordeau. 1898. I'. L. II I, pp. 35 102). Gebmarn. - Statistique forestière du Dép. du Cantal. 1889.

(inlot, Dr. - Observations sur quelques plantes critiques du centre de la France (Riev. de bot., Toulouse, 18920).

(innon, Dr' ['. - Comparaison de la flore alpine l'Auvergne avec celle du Jura (Ass. Franç. des Sc.) Congrès Besançon, p. 239, 1893.

GoNon b'Artemare. - Etudes sur les plantes qui croissent autour des eaux minérales et recherches sur la présence de l’iode dans les eaux min. de l'Auvergne (Thèse 1896).

Goxon n'Artemare. - Matériaux pour la llore d'Anvergne (Rev. Sci. Bourb. et centr. Fr., 1889).

Gowon D'Artemare. - Matériaux pour la llore d'Auvergne (Rev. Sci. Bourl). et cent. Fr., 1890-1892).

Gixer, J. E. - Flora norv. 2 pts. Nidrosia 1766 1772. fol. c. 12 tabl. Ilamberg. - Om Slogarnes Inllytande pa Sveriges Kilimat (Nordiska Blihln, 1889).

II Aвтила, C. - llandbok i Scandinaviens flora.

11 Alitunn. - Skandinaviens Ormbunkar, 1889.

Hecier. - Sur l'intensité des couleurs el les dimensions considérables des fleurs aux hautes altitudes. (Bull. de la Soc. bot. de France. Tome XXY, 1883.

II EcFkEY. - The vegetation of Europe, 1852.

Hexig. - Botanik, 1901. (Beijers fürlag).

IIERB.L1, Josepn Fr. - Recherches sur les Diatomées des calcaires tertiaires de l'Auvergne et sur l'origine de ces terrains (Rev. sc. du Bourb. et centre de la France, t. X, pp. 221, 30, 1897).

Inkzbacd, Josepn Fr. - Les Diatomées d'Auvergne (Rev. d'Auv. 1 vol. 232 p. 6 pl. Paris-Clermont, 1893). 
IIfribaud Josepil Fr. - Le P'uy-de-Dôme et le Cantal ou tab. comp. des pl. vasculaires el spontanées de ces deux dép. (Assoc. franc. aranc. des Sciences, p. 41I, 1876).

Jleriraco Josern lir. - Lettre à M. Malinvaud sur la découverte du Woodsia hyperborea recueilli dans le Cantal à une alt. de 1600 in. (Bull. soc. bot. de France, t. XXYT, p. 205, 1881).

IIeribaci Josepil Fr. - La flore d'Aurergne en 1901 (Bull. soc. bot. de france, t. XLVIII, 190I).

Horfarans. - Resultate der vichtigsten pflanzen-phänologisehen Beobachtungen in Europa nebst einer Früllingskarte, 1885.

IloffMann uxp lnce. - Beiträge zur l'hänologie, 188'.

II ue, Abisé. - Lichens du Cantal et de quelques dép. voisins (Bull. soc. bot. de Fr., t. XXXIV, 1890).

IIult. - Bildatlas örver Yäxtriliet 189' (Fritzes Bohkandel).

Ilut. - Recherches sur les phénomènes périodiques des plantes. (Upsala 1881).

IÏ̈GRELL. - Botanikens Historia, 1886.

Jonunssox, $K$ - IIufruddragen af Gotlands vïxttopografi och viixgeografi. Stockih, 1897.

Johansson, K. - Gotlands fanerogamer orh ormbunkar. Sthlm, 1889.

KELLER. - Die Blüten alpiner Pllanzen, ihre Grüsse und Farbenpracht, Basel, 1887.

Kernerr. - Phanzenieben. A. 11 p. $501 \mathrm{f}$.

Kenner A. V. - Die Abhängigkeit der Pllanzengestalt von Kilima und Boden. (Tagebl. d. 4:3 Vers. deut. Aerzte un Naturfor). Innsbruck, 1869.

KinLmavs. - Pllanzenbiologische Studien aus rus. Lappland. II elsingfors, 1890.

Limlmaxs. - Beob. üb. d. period. Lirschein. d. Pflanzenlebens in Finland. Ilelsingfors, 1886.

Kinlansw. - Pllanzenbiologische St. aus russ. Lappland. s. A. aus $\Lambda$ cta Soc. pro fauva et flora fen. t. VI, 1890.

Kúliman F. K.- Aus dem Leben der Polarpllanzen. Leipzig, 1885. Liselumas, F. K. $-\Lambda$ us dem Pflanzenleben wïhrend des Winters in Meere an der Westküste von Schweden. (Bot. Centralbl, Bd 26) 1886 .

KJellanan F. K. - Aus dem Leben der l'olarpflanzen in: Nordenskjöld Studien. Leipzig, 1883. 
Kifsumin F. li. - Norra ishafvets algflora. Vega exped. vetensk. iakt. (Bd. 3, 1893).

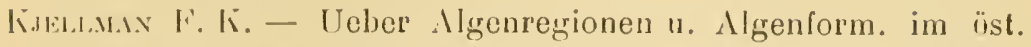
skagerak (Bilıang till K., sv. vet. al. handl. hd. 5. n" 6, 1878).

K.llt.M. F. K. - The alg. of the arctic sea. (K. sv. vet. ak. handl. $n$ y fiiljd 20 bd. 1882-188:3).

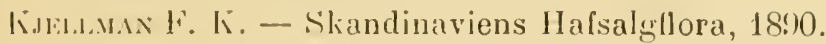

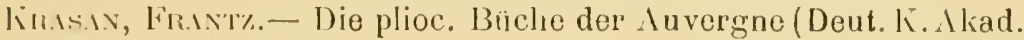

IViss. Wien 18:9).

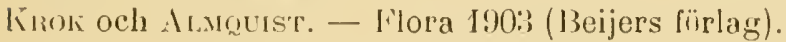

kimk och Almovest. - Srensk flora II, Kryptogamer. StockI 1898. Kuckick, P'. - Berträge zur lienntuiss der Meeresalgen 1897.

Küresex. - Géogr. Verber. d. Nadelhülzer im europ. Russland (voir G. J. XIII 319 แ० 156).

lï̈rex. - Geogr. Verbr. d. Ilolygewïclise d. europ. Russl. u. d. Kaukasus. P'itersb. 1888-1889.

L.inotre M. - P'rodrome de la llore du l'latean central. (Mem. acad. des sci. lett. arts. Clermont-Fil 1875-78 799-80).

L.motes M. - Extrait d'une letlre à M. Malinvaud sur ia découverte par les fres Iléribaud el Gatien du Sasifraga hieracifolia. (Bull. soc. bol. d. Fr. 1. XXVI, p.91) 1879.

L.MY DE L Curapter. - Les lichens du centre de la France (Jour. d'hist. nat. de Bordeaux et (u S.-O., 22 ann. $n^{n}$ y) 1883.

L.I.re. J. - Handb. i d. Danske flora. Kjübenh, 1888.

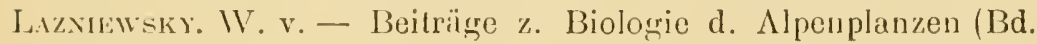
82. 1896.

LECOQ eT L.мнотTE M. - Catalogue raisonné des plantes vasc. du Plateau centr. de la l'rance comprenant l'Auvergne, le Velay... (Ann. Sci. lill. el ind. de l'Auvergne. Clermont-Fd. in $8^{\circ}$ ).

Lecor, II. - Etude sur la géographie de l'Europe et en part. sur la vég. du Plateau centr. d. la Firance. 9) vol. Paris 185.

Liraı.. - Quels sont les caractères dist. qu'on pourrait sign, dans la flore de certaines rér. du I'l. centr'. de la Fr. (Cong. sci. de Fr. XXII pp. $3633(; 7.185 \%)$.

Lıı.ı, N. - Flora ricanica. 2 uppl. 2) vol. Sitockh. 1870).

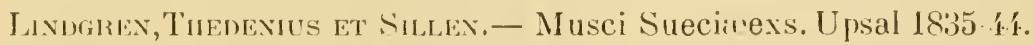

Lismix. - Bilder ur Nordens Flora.

Lixnmix, C, $\Lambda$, M. - Blïten und Bestaubungseinrichtungen im scandinavischen Hochgebirge. (Bot. Centralbl, Bd, IXX 1887). 
LINNÉ, C. - Flora Lapponica (in-8 ${ }^{\circ}$ et 12 tab]. 1737).

Linvé, G. - Flora Suecica (8 fig. Sthlm 175\%).

Lixis, C. - Lachesis Lapponica (Tow in Lappland, now first publ. by J. E. Smith. 2 vols. Lond. 1811).

Lixí, C. - Flora Dalecarlica. Opus ined. (Örebro 187:3).

Lorexz, R. - Physik. Verh. und Verth. d. Org.im Quarnerischen Golf. Wien 1863.

Lixgbie, II. Ch. - Tent. Ilydrog. Danico cont. omnid IIydrophyta, Cryptogama Dan. IIols. Faer. Isl. Grœnl. (Kjöbenhaven 1819).

LitTkens. - Om Svenslia Ogräs, 1885.

Mindendorfs A. V. - Die Gewächse Sibiriens. Bd IV 1 th. 186 i.

Moxvien L. - Obs, de bot. et d'hist. nat. faites en Auvergne (Mém. de l'Arad. des siciences) 1140.

Mülter, Henr. - Alpenblumen, ilıre Befruchtung durch Ins. n. ihre Inpassungen an dieselben. Leipzig 1881.

Natnorst. - Geologi, 1901. (Beijers förlag).

N.tuorst, 1. G. - Studien über die Flora Sipitzbergens. Engler's Bot. Jahrb. I. Systematik... Bd IV 1883.

Neunas, L. M. - Sveriges flora. Lund 1901.

Nol, F. - Ueber die Kultur von Meeres Algen in Aquarien. 1892.

Nonu.1, M. J. - Florie arctica Norvegie. (Christiania Vedensliabsselsliabs Forhandlinger for $\left.1893 \mathrm{n}^{\prime \prime} 16\right)$.

Níander D. V. - Note sur les licliens rec. en Auvergns pend. 1. sess. extraord. d.l. Société en juillet 1850 (Bull. soc. bot. d. Fr. t. III) 1856.

Nyman C. F. - Sveriges fanerogamer, Örebro 1868.

Nimix G. F. - Svensk fanerogamllora, Sthlm 1873.

Ostexfeld, C. II. - Flora arclica (Copenhagen. Det nordiske För$\operatorname{lag}, 1902$.

PAx. - Ursprung der europ. IValdbäume 1886.

Rayes J.-B. - Généralités sur cert. rel. de la flore du Cantal avec la topog. et la géog. de ce dép. (Bull. soc. bot. d. l. Fr. T. XXVI, p. 9. 1879).

RAnes J.-B. - Rapp. sur l'exc. paléontologique faite par la Soc. le 27/7 1879 en Auvergne (Bull. de la Soc. bot. de Fr. t. XXVI, 1879).

RANvier. - Obs. sur la distrib. des espèces régétales phan. dans le 
Plateau et les deux versants de la Marg. (Bull. soc. agric. de Mende. 1. VIII p. 383-411, 1858).

Ratikiner, C. - Danske blomsterplanters naturhistoria Bd. 1. Monocot. Kjö̈benh. 1895-99.

Reixke. - Algenflora d. West. Ostsee. Bericht der Kom. zur wiss.

Untersuchung d. deut. Meere, 1889.

RosENdahL. - Botanik 1903 (Sandhergs förlag).

Roze et Bocnier. - Contr. à l'étude mycologique de l'Auvergne.

(Bull. soc. bot. de Fr. t. XXVI, 1879).

Rubexsox. - Temperatur - orh Fuktighetsförhällanden, Vetensk. Ak. Handl. $32 \mathrm{irg}$.

Saporta DE. - Forêts ensevelies sous les cendres érup. de l'ancien volcan du Cantal, observées par M. B. Rames et conséquences de cette découv. pour la connaissance de la vég. dans le centre de la Fr. à l'ép. pliocène. (Compte rendu acad. sc. t. LXXXI p. p. 290 294) 1873.

SaPORTA DE. - Sur les carac. prop. à la vég. pliocène à propos d. decouvertes de M. B. Rames dans le Cantal (Bull. soc. géol. Fr. sceance du 17 fév. 1870).

Schlagerström, Aug. - Conspectus vegetationis uplandica (Upsalim).

Schagerström, Avg. - Plantie cotyledonie Parøcie roslagie Bro. (Upsaliae).

Scnexz. - Smảlands flora. Wexjö 186 '.

Scminper F. WT.- Ueber Schutzmittel des Laubes gegen Transpi-

ration. (Sitzung d. Kön. preuss. 1 kad. der Wissensch. zu Berlin 1890$)$.

Scnübeler, G. - Die l'llanzenwelt Norwegens. Kiristiania 1875.

Ścrüneler, G. Växtlivet i Norge, 1879. - Norges vïxtrike, 1888. - P'flanzengeog. Karte Norwegens 1876.

Ścnüreleli, C. - Die Kulturpllanzen Norwegens. Kiristiania 1862. Schüneler, G. - Die Pflanzenvelt Norwegens, 2 Thl. (Christ. 1875, 4 m. 80 Illustr. n. I6 Karten).

Scrulz, ג. - Entwicklungsgeschichte d. Phan. d. skand. IIalbinsel. gr-8 Stuttgart 1901.

Sü̈straxd M.G. - Calmar lïns ocrh Ölands flora, Calmar 1863.

Soulavie (Giraud). - IIistoire nat. d. I. Fr. mérid. 7 vol. in- $8^{n}$.

Paris. 2 partie. Les végétaux. 1783, 1 vol.

Ströxfelt, II. F. G.- Islands Kärlväxter, betraktade frản växtgeo- 
grafisk och floristisk synpunkt. (Öfversigt af Kongl. Vetenskaps Akademiens Förhandlingar 1884. No 8, Stockholm).

Suxdbera. - llistorisk Statistisk llandbok, Sthlm 1900.

Svedmaris. - Geologi (Fritzes Bolffïlag) Sthlm 1894.

Svenson. - Flora öfver Sveriges liulturvïxter, 1893. (Norstedts förlag).

Saye, Car. - Synopsis Florie Gothlandicir (Upsalioe 1837).

Tuedenius, k. F. - Upplands och Sïdermalands flora. Sthlm 1871.

Thedenius Fr. - Stockholmstraktens Fanerogamer och Ormbunkar: (Stockholm, 1850. Tryckt hos Joh. Beckman).

Trollus Magnus Adolf. - Om Westerảstrakten i botaniskt afseende (Stockholm tryckit hos E. Westrell 1860).

Тӥгхевонм. - Geologi 190? (Norstedts förlag).

Vinduls F. - Terrains gran. agric. d. centre de la Fr. J. I. Les agents nat. de la vég. ; le sol et les engrais, les champs, les pics, les bois. Paris in 120, 30\% pages, 1885 .

WAGNer A. - Zur Kentniss des Blatthaues der Alpenflanzen und dessen biol. Bedeut. (Sitz. d. Wiener Akad. Bd. CI. Abt. I 1892).

Wanlströs, E. - Plantarum vascularium in regione, Telgae borealis (Upsaliae).

Wh ahenderg, G. - Flora lapponica, 1812.

F'lora Upsaliensis. Upsal. 1820.

\section{- $\quad$ Flora Suecica 2 vol. Upsal. 1824-26,}

WAILstedt. - Läroboli i Botanik, Lund 1873.

Warming, E. - Lehrbuch der ökologischen pflanzengeog. (Deutsch von linoblauch, 1896).

Warming, E. - Om Grönlands vegetation (Mit. franz. Résumé, Kjüb 1888).

Weibult. - Mineralogi och Geologi, Sthlm 1891.

Westerberc. - Geologi (Beijers förlag) 1901.

WESSEN, IoH. - Plante cotyledoneae in Paroecia Ostrogothiae Karna (Upsaliae) 1838.

Zetterstrot J.-E. - Kinnekulles Phanerogamer och Ormbunkar (Aftryck fràn Botaniska Notiser för àr 1851).

ZetTerstedt J. F. - Om vegetationen i de höglïndtaste trakterna af Smaland (Kongl. Srenska Vetenskaps-Akademiens Handlingar Band 6, № 2), 1865. 


\section{TROISIÈME PARTIE}

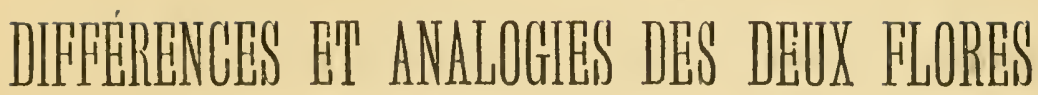

L'exposé qui précècle doit être terminé par la recherche des analogies et des différences que la flore dn Massif Central possède avec les espèces analogues du Massil Scandinave, en nous basant sur l'examen morphologrique extèrieur et sur les dillérences histologiques des mêmes lormes empr'untées aux deux llores el comparées entre elles.

Nous avons, dans cette comparaison, rapproché le plus grand nombre d'espèces possible : nous arons constaté que beaucoup d'espéces étaient pour ainsi dire immuables, que d'autres présentaient des différences très légères, que certaines enfin marquaient nettement des dilférentiations précises. G'est sur ces dernières qu'ont porté plus particulièrement nos recherches, car', en exagérant les caractères, elles rendent plus sensibles les dillérences et permettent d'en rechercher' les raisons physiologiques tant dans l'état atmosphérique que dans la composition du sol.

Les formes qui nous ont particulierement frappé sont les suivantes. Nous les indiquons avec la station où ont été recueillis les exemplaires observés:

\section{Juncus alpinus Vill.}

Bengtstorp, Vestmaland 4/7 1878 Plomb du Cantal, août 1896.

$$
\text { Luzula spicata I). C. }
$$

liongsvold, Dorre (Norvège) 24/8 Puy-de-Dôme, juillet 1889 $[1882$

Juncus filiformis $L$.

Stockholı, juillet $1880 \quad$ Plomb du Cantal, août 1887

Carex vaginata, Tausch.

Torp, Medelpad 23/7 1875

Mont-Dore, 2/8 1880 
Saxifraga hieracifolia $\mathrm{W}$. et $K$.

Sjüdalen, N. Vaag., 4/8 $1870 \quad$ P'as-de-Roland, Cantal 25/7 1903

Salix herbacea $L$.

Laxfjellet, Laponie 17/7 $1869 \quad$ Mont-Dore, août 1902

Salix phylicifolia $L$.

Kongsvold, Dovre (Norvège) 6/7 Mont-Dore, août 1903

[1882

Salix lapponum $L$.

Kongsvold, Dovre (Norvige) 12/7 Mont-Dore, août 1903 $[1882$

Gnaphalium nor'egicum Gum.

Fjällnäs, Herjedalen 20/8 1893 Bois du Lioran, Cantal, août 1884

Empetrum nigrum $L$.

Upsala, Uppland, juin $1875 \quad$ Puy-Mary, Cantal, août 1903

Serratula tinctoria $L$.

Godegảrd,Ostrogothie,juillet 190\% Mont-Dore, août 1894

Vaccinum vitis idaea $L$.

Ör, Dalsland 30/5 1889

Puy-llary, Cantal, août 1892

Ajuga pyramidalis $L$.

Upsala, Uppland, juin 1873

Puy Mary, Cantal, juillet 1897

Pulsatilla vernalis Mill.

Säter's, Dalécarlie, juin $1890 \quad$ Mont-Dore, juin 1901

Galium boreale $L$.

Ekerö, Stockh. län 26/6 $1885 \quad$ Plomb du Cantal, aout I89I

Circaea alpina $L$.

Sl. Rüstảnga, juillet 1887 Puy-Mlary, Cantal, août 1900

Dryas octopetala $L$.

Kongsvold, Dovre (Norvège), juil. Puy Mary, Cantal juillet 1901 
Sargina Linurea, Presl.

Kongsvold, Dovre (Norvige) 25/7 Plomb du Cantal, aout 1857

[1882

Bartsia alpina $L$.

Areskutan, Jemtland 18/7 [1877 Puy-Mary, Cantal, août 190 I

Saxifragaoppositifolia $L$.

Kinudshüe, Dovre (Norvige) 24./7 Pas-de-Roland, Cantal, juillet 1900 $[185 \%$

Selaginella selagonoides L. Selaginella spinulosa Br.

Rüttvik, Jalécarlie, juillet 1902 Monts-Dores, Puy-de-1)òme, aout

$[1875$

Aspidium Lonchitis Sto.

Areskutan, Jemtland 18/7 1890 Pas-de-Roland, Cantal, août 1903

Lycopodium Selago L.

Skün, Medelpad $4 / 71876$

Puy-Mlary, Cantal, août 1903

Woodsia hyperborea R. B.

Areskutan, Jemtland, juillet 1885) Rochers phonolitiques, Cantal [aout 1901

\section{Hyprnum callichroum.}

Godösund, 16/7 1875

Pas de-Roland, Cantal, août 1882

Dans cette liste, nous allons passer en revue les formes les plus spécialement différenciées et remarquables.

\section{$1^{\circ}$ DIFFÉRENCES MORPHOLOGIQUES}

Salic phylicifolia. - Les feuilles de l'espèce scandinave, sont plus grandes et beaucoup plus pointues. Elle lleurit un mois avant celle du Massif Central.

Salix lapponum. - La tige de la plante du Nord est couverte de poils, tandis que celle du Massif central n'en 
possède que très peu; il en est de même pour les feuilles qui, en outre, sont plus grandes et présentent des pétioles phis longs.

Galium boreale. - L'espèce scandinave est beaucoup plus développée que le type correspondant du Massif central. Les entrenœuds de la tige sont par suite bien plus écartés. Quant aux feuilles, si leur nombre est moindre, elles sont plus larges et mieux développées. Les lleurs sont très abondantes et leur corolle s'étale trois mois avant la lloraison dn type correspondant du Massif central.

Circra alpina. - Dans le nord celte espece est trois fois plus grande que la plante du Massif central; elle est aussi beaucoup plus trapue. Les feuilles sont plus profondément dentées et elle developpe ses organes reproducteur's bien avant l'espèce française.

Ajuga pyramidalis. - Comme le type précédent, elle est beaucoup plus développée que son correspondant du Massif central. La racine, plus robuste, porte davantage de radicelles, les feuilles, plus nombreuses, sont aussi mieux développées et plus épaisses; enfin la lloraison se lait mn mois plus tôt.

Serratula tinctoria. - On peut en dire autant de cette espèce.

Empetrum nigrum. - Présente les mêmes différences.

Gnaphalium norvergicum. - Une taille plus considérable s'observe aussi dans cette espèce, mais en outre la tige a des poils nombreux et elle est aussi plus épaisse; il en est de même des feuilles qui sont aussi plus nombreuses. Quant aux fleurs, leur nombre est plus élevé et elles sont aussi plus serrées.

\section{$2^{\circ}$ DIFFÉRENCES HISTOLOGIQUES}

La cornparaison des coupes de tiges, racines et feuilles des mêmes plantes, recueillies sensiblement aux mêmes périodes 
de végélalion, le choix d'axes de mème igge a élé fait avec le plus grand soin et c'est sur l'examen de longues et nombreuses séries de conpes transversales et longiludinales que nons établissons nos conchusions qui sonl les suivantes :

Les plantes d'Auvergne sont munies dans leur appareil végélatif aérien, de zones collenchymateuses et selérenchymaleuses lormant un stéréome protecteur lrès accentué.

Le développeneul du stéréôme se retrouve dans la racine avec des caraclères de même ordre.

Dans les plantes scandinaves on olsserve inversement une tendance à l'accroissement des parenchymes à paroi mince qui enveloppent la lige, la racine et mème la leuille.

Pour fixer les idées sur ces points, nous choisissons dans nos coupes celles que nons allons décrire el que nous reproduisons, à la chambre claire, dans les plantes qui accompagnent ce travail.

\section{PLANGHE I}

La tige du Bartsia alpina d'origine scandinave, comparée avec la mème tige d'origine aurergnate, montre la tendance à la formation des couches incrustées dans les plantes françaises.

La forme scandinave (fig. 1), nous offre sous l'épiderme cutinisé un parenchyme cortical formé de cellules polygonales ou rectangulaires, sans méats interposés. Cos cellules se rapetissent, s’applatissent et se condensent dans la profondeur, mais sans épaissir leur paroi, formant un anneau endodermique à assises superposées. La formation libérienne est épaisse, c'est du liber mou sans trace de sclérenchyme fibreux, il passe a un cambium et, au-dessous, le bois s'affirme par des vaisseaux ligneux dispersés dans un parenchyme central à parois minces cellulosiques.

La forme anverirnate (fig. 2), met en évidence des caractères différentiels très accusés : réduction des formations parenchymateuses, incrustation des parties restantes. La réluction du parenchyme cortical s'affirme par une épaisseur moindre, mais surtout par une sorte de dissociation cellulaire. Elles sont arrondies, séparées par de longs méats, s'unissant mè̀me en lacunes plus étendues.

Une couche endodernique unique limite le parenchyme on de- 
dans, et eontre elle repose un anneau de fibres libériennes très puis. sant. C'est l'apparition du stéréome caractéristique. Au-dessous, un liber mou à tubes criblés, la zone cambiale, et le bois, dont les vais. seaux sont enveloppés par des fibres incrustés qui complètent le stéréome de la tige.

Les grands méats du parencliyme cortical, la zone scléreuse cor ticale, la zone scléreuse ligneuse donnent au type du Massif central des caractères nettement tranchés qui pourraient permettre de l'opposer conme une car. Arvernica au type scandinave.

Ces caractères se retrouvent dans la racine avec la même netteté. Dans la forme scandinave (fig. 3), l'endoderme conserve à tout âge ses caractères primitifs, ses cellules à denticulations latérales donnant une projection punctiforme, de plus, le parenchyme du cylindre central reste à parois minces.

Dans la forme auvergnate (fig. 4), l'endoderme s'épaissit et s'incruste: de plus, la partie centrale du cylindre médullaire devient scléreux et les faisceaux du bois se prolongent au milieu d'éléments allongés présentant sur la coupe la forme polygonale avec épaississement de la paroi.

\section{PIANCHE II}

La série des saules se prête aux mêmes observations.

La tige de Salix lapponum provenant de Scandinavie (fig. 1 et 2) offre, sous un épiderme à corticule mince un épais parenchyme cor. tical qui passe insensiblement à un liber mou bien caractérisé, puis vient un mince cambium et un corps ligneux très fortement incrusté formé de fibres et de gros vaisseaux disséminés.

La mème tige provenant des récoltes des Monts Dores (fig. 3 et 4) se distingue de suite par ses couches épaissies de protection. La cuticule est plus épaisse et sous l'épiderme on constate un hypoderme formé par un collenchyme ayant une épaisseur moyenne de dix cellules. Le parenchyme cortical à cellules arrondies se termine contre des îlots sclérenchymateux de fibres libériennes qui protègent le liber mou. Le corps iigneux, à peu près semblable, offre une plus grande épaisseur des parois incrustées des fibres ligneuses.

Salix phylicifolia se prète aux mèmes observations. Dans la forme auvergnate (fig. 5) on retrouve les mêmes îlots de fibres libériennes qui manquent dans la forme scandinave.

Ce qui est vrai pour la lige et la racine est confirmé par l'étude de la feuille des Salix. 
Dans la forme scandinave (fig. 6), nous constatons un plus grand développement du parenchyme; c'est à peine si un anneau de deux rangs d'éléments sclérenchymateux surmonte la partie ligneuse du faisceau.

Dans la forme auvergnate (fig. 7), nous complons deux bandes sclérenchymateuses supplémentaires, de plus la bande qui correspond au bois du laisceau s'épaissit el se fusionne avec ses fibres incrustées.

\section{PLANCHE III}

Comme les Salix, Gnaphalium norcegicum met en relief les mêmes données. L'épaississement du parenchyme, la cohésion de ses cellules s'affirme dans la forme scandinave (fig. I) comparée à la fur me auvergnate (fig. 2).

Dans celle espèce les caractères différentiels des feuilles sont encore plus nettement affirmés que dans les Salix.

Dans la forme scandinave (fig. 3) le parenchyme prend un développement entre l'épiderme el le faisceau qui esl le double de celui qu'il présente dans la forme auvergnate (fig. 4). Au microscope on se rend compte de l'allure si différente de ce parenchyme dans les deux formes, scandinave (fig. j) el auvergnale (fig. (i)).

L'épaisseur de l'ønneau qui entorre chaque faisceau est en raison inverse du développement du parenchyme, et avec l'épaississement de l'anneau dans la forme auvergnate. coïncide une incrustation plus intense de la parois épaissie des éléments scléreux.

\section{JLANCIIE IN}

Un dernier exemple, Saxifraga opposilifulia, montre la généralisation de ces données à l'ensemble des espèce; observées. Dans la forme scandinare ( $(\mathrm{gg} .1$ ), sous la couche de liège qui limite la lige on ne trouve que du parenchyme ordinaire aussi bien dans la zone corticale que dans le cylindre central.

Dans la forme auvergnate (fig. 2), au contraire, un anneau sclérenchymateux épais trace la limite entre le parenchyme cortical et le liber du corps libero-ligneux. En même temps, le parenchyme cortical se réduit. Ce sont toujours les mêmes rapports, les mêmes phénomèues.

La racine de Carex vayinata complète nos observations sur la racine de Rartsia alpina. A un faible grossissement la coupe de la forme scandinave (fig. 3) comparée à celle de la forme auvergnate 
(fig. 1), met en relief le développement considérable du parenchỵme cortical et la réduction correspondante du cylindre central.

Au microscope, cette différence est rendue plus caractérisée par l'étude des groupements cellulaires intérieurs. De grandes lacunes aérifères sont creusées dans le parenchyme, limitées du côté du cylindre central dans la forme scandinave (fig. j) par des couches de cellules superposées qui se réduisent, dans la forme auvergnate (fig.6), d̀ une seule assise de cellules superposées à l'endoderme. Le point noir marqué sur les firures marque le centre du cylindre central. On peut se rendre compte du développement presque double de ce cylindre dans la forme auvergnate. L'endoderme est épaissi dans les deux cas, le parenchyme du cylindre devient scléreux dans toule son épaisseur.

Ces exemples pris, nous pourrions dire presque au hasard, dans les nombreuses séries de nos préparations sont confirmés par toutes nos ubservations sur les espèces désignées dans la précédente liste, et nous pouvons affirmer les conclusions suivantes:

Les plantes scandinaves ont donné plus de puissance aux parenchymes périphériques, dans la tige et la racine, le parenchyme cortical est sensiblement d'un tiers plus développé que dans les espèces correspondantes d'A uverg.ıe.

C'est par la multiplication des couches superposées que ces plantes ont assuré la protection au cylindre central. Dans la feuille le parenchyme inter-épidermique qui entoure les faisceaux présente la même exagération de développement. Mais si les cellules multiplient leurs assises elles n'ont pas de tendance à épaissir leurs parois pour former des collenchymes et à les incruster pour former des sclérenchymes.

Les plantes auvergnates, au contraire, réduisent pour ainsi dire leurs formations parenchymateuses, mais, en revanche, elles modifient profondément l'épaisseur et la structure des parois, de là des formations hypodermiques de collenclyme, des îlots ou des anneaux continus de sclérenchyme constituant une stéréome interne large. ment protecteur. Celte disposition est certainement dùe à des conditions déterminantes du milieu ex lérieur qu'il nous reste à déterminer.

\section{$3^{\circ}$ CAUSES DE CES DIFFÉRENCES}

Les dilférences que nous venons de noter, soit dans l'aspect exterieur, soil dans la slructure interne, des plantes 
scandinaviennes comparées an mimes espèces dı Massif Central penvent ètre altribuées i direrses canses.

Ces canses penvent etre recherches parmi les agents physiques extérieurs et anssi s'altribuer en partie au soussol. Parni les premieres, les principales sont : la température, l'éclairement et l'humidité de l'air. Parmi les secondes la comprosition du sol, sa nalure chinnipue ef la yuantité d'eau yüil peut renlermer.

Si la température de la presqu'île scandinave est un peu moins élevé yue cellèdu Massil C'entral; l'écluirement est ici un facteur rqui doil agir plus spécialement. M. Dufour a en eflet montré que les leuilles sont plus épaisses lorsqu'elles sont plus éclairées; ce fait a été vérifié par M. Bomnier, or la plupart des esłèces scandinaves offrent des fenilles épaisses et l'on peut penser' que l'éclairement en est une des causes.

L'humidité de l'air n'est pas la même en Scandinarie et dans le Massil Central. Si l'on s'élève sur les sommets du centre de la France l'air devient de plus en plus sec, tandis que si au contraire, on se dirige vers le Nord au fur et à mesure que la latilude est plus élerée l'air devient aussi de plus en plus humide.

On sait, el MM. Lollielier et Bonnier ont démontré expérimentalement que, dans un air plus humide la structure de la tige et de la feuille se modifie. D'une manière générale la plante qui est dans un air plus humide est moins diflérenciée dans tous ses tissus. Dans les feuilles le tissu en palissade diminue ou tend même à disparaitre, les lacunes sont plus abondantes, la cuticule moins épaisse. Dans les tissus de la tige on de la feuille elle est moindre.

Nous trourons toutes ces différences dans les plantes scandinaves observées par rapport à celles du Massif Central. Nous pourons donc les attribuer en très grande partie à l'humidité plus considérable de la région du nord.

Les modifications, que présentent les racines peuvent être attribuer en partie à la nature du sous-sol ; tandis qu'en 
Scandinavie ce sont parlout des roches primitives qui dominent, les roches dans le Massif Central sont des plus variées de plus, l'humidité du sol, qui est plus considérable en Suède et Norvège que dans le centre de la France joint son ellet au précédent.

L'ensemble de toutes ces causes et en particulier l'éclairement ef l'humidité de l'air impriment anx llores comparativement étudiées leur laciès propre, qui résulte de leur adaptation au milieu physique dans lequel elles vivent.

Il en résulte qu'un certain nombre de plantes de la flore glaciaire ont pu se maintenir sur le sol de l'Auvergne lorsque des conditions nouvelles modiliaient profondément le climat du Platean Central. La grande majorité des espèces glaciaires a disparu et celles qui ont persisté n'ont pu le faire qu'en modifiant leur structure dans le sens que nous avons indiqué. Elles sont devenues en queique sorte des variétés avernica des espèces primitives qui pour nous, se présentent en Suède avec leurs types initiaux.

Telles sont les relations que l'on pent établir entre la flore de ces deux Massiĺs éloignés qui sont un des points les plus intéressants de l'étude botanique des deux régions.

Lu, bon à imprimer :

Clermont-Fd., le 21 Juin 1905.

Vu et approuvé :

L.e Professeur de Botanique de la Faculte des Sciences, Le Doyen de la Facultedes Sciences, J. POIRIER. Dr PAUL GIROD.

Vu, et permis d'imprimer :

Clermont-Ferrand, le 21 Juillet 1905.

Le Rectęur de l'Académie de Clermont-Ferrand,

A. COVILLE. 

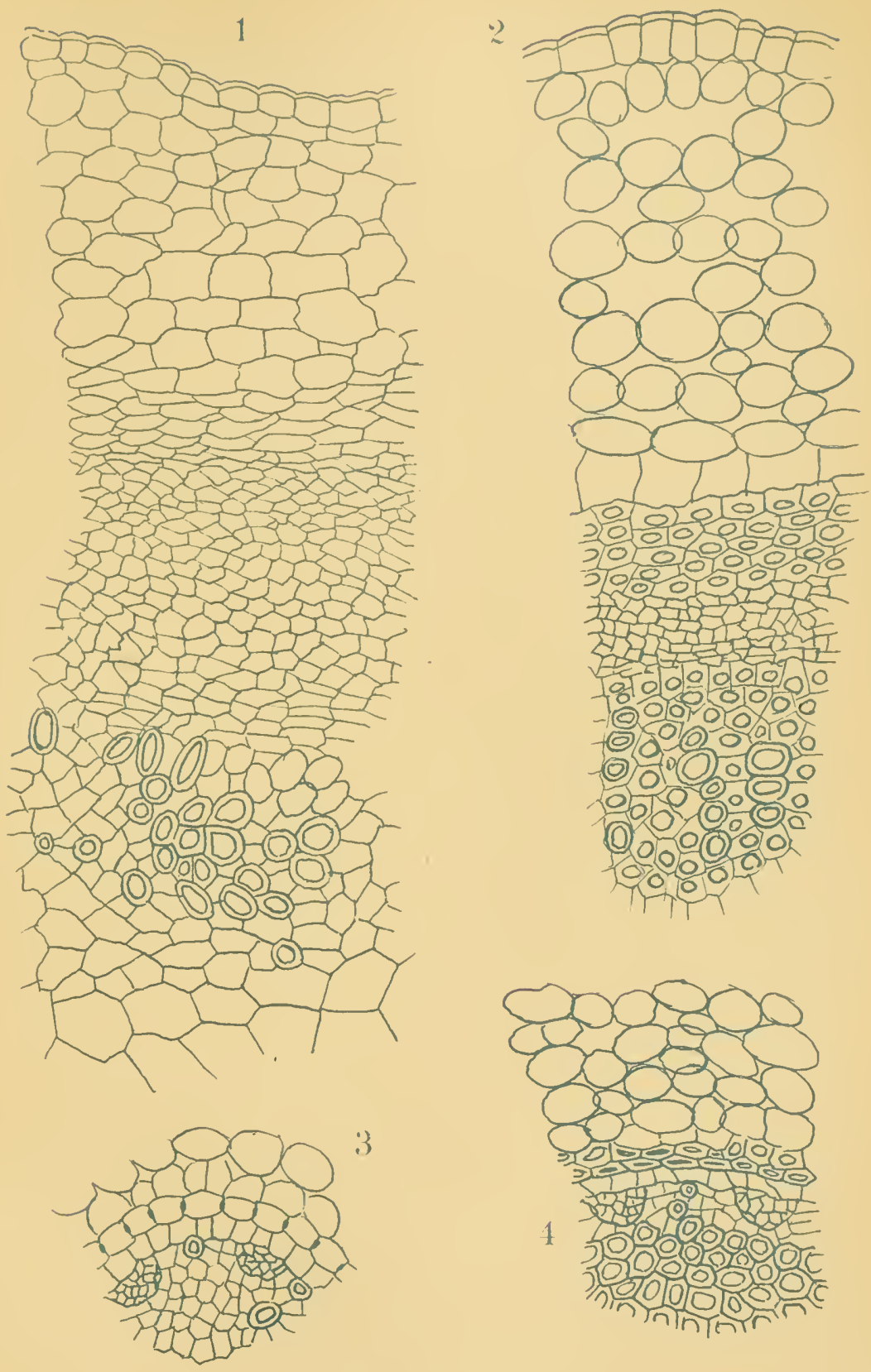

1

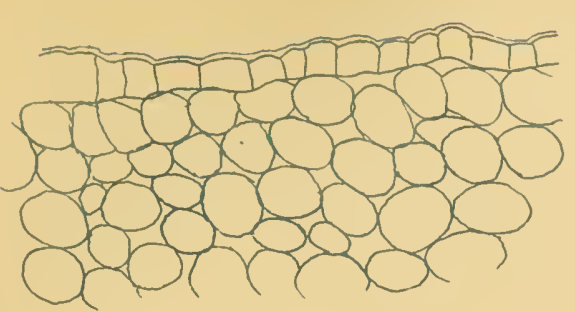

:3

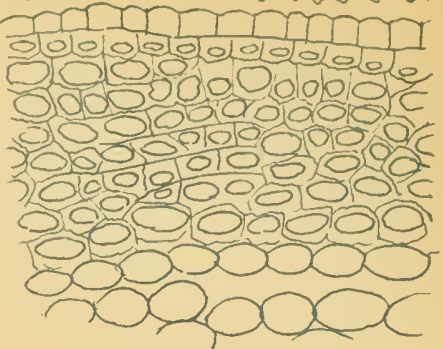

2

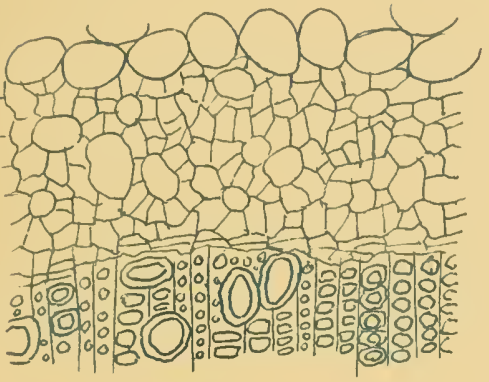

6

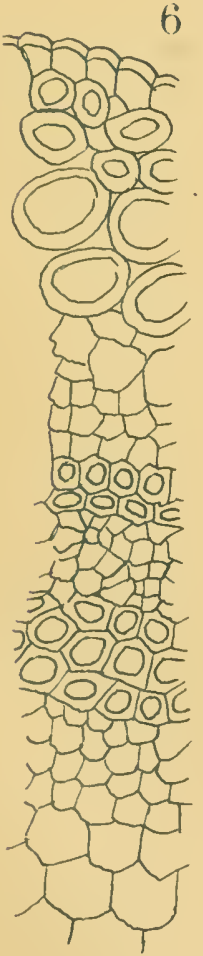

7

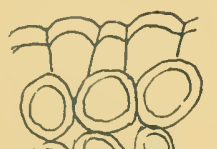
12 o) ctom ror rut (a) ogo 0 o moro 0 001000 10000 0000 0100 ont onites inis 000 ono (1) (1)

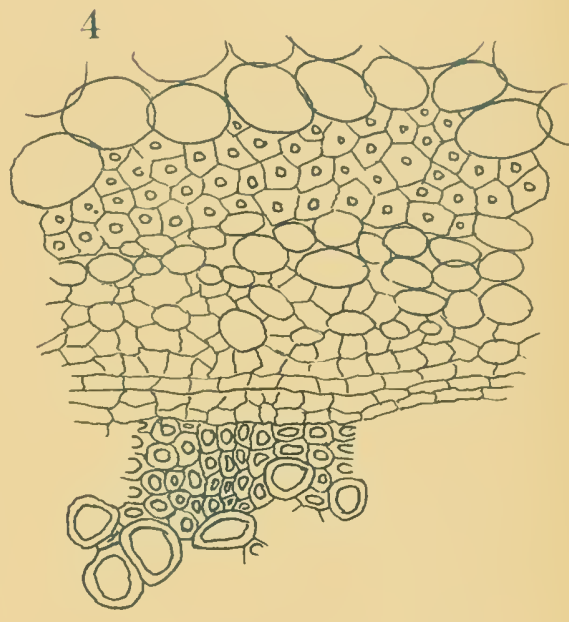

5

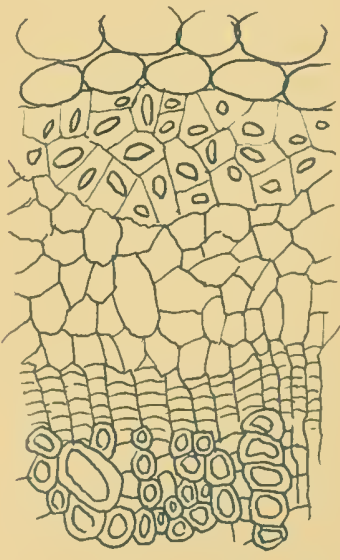




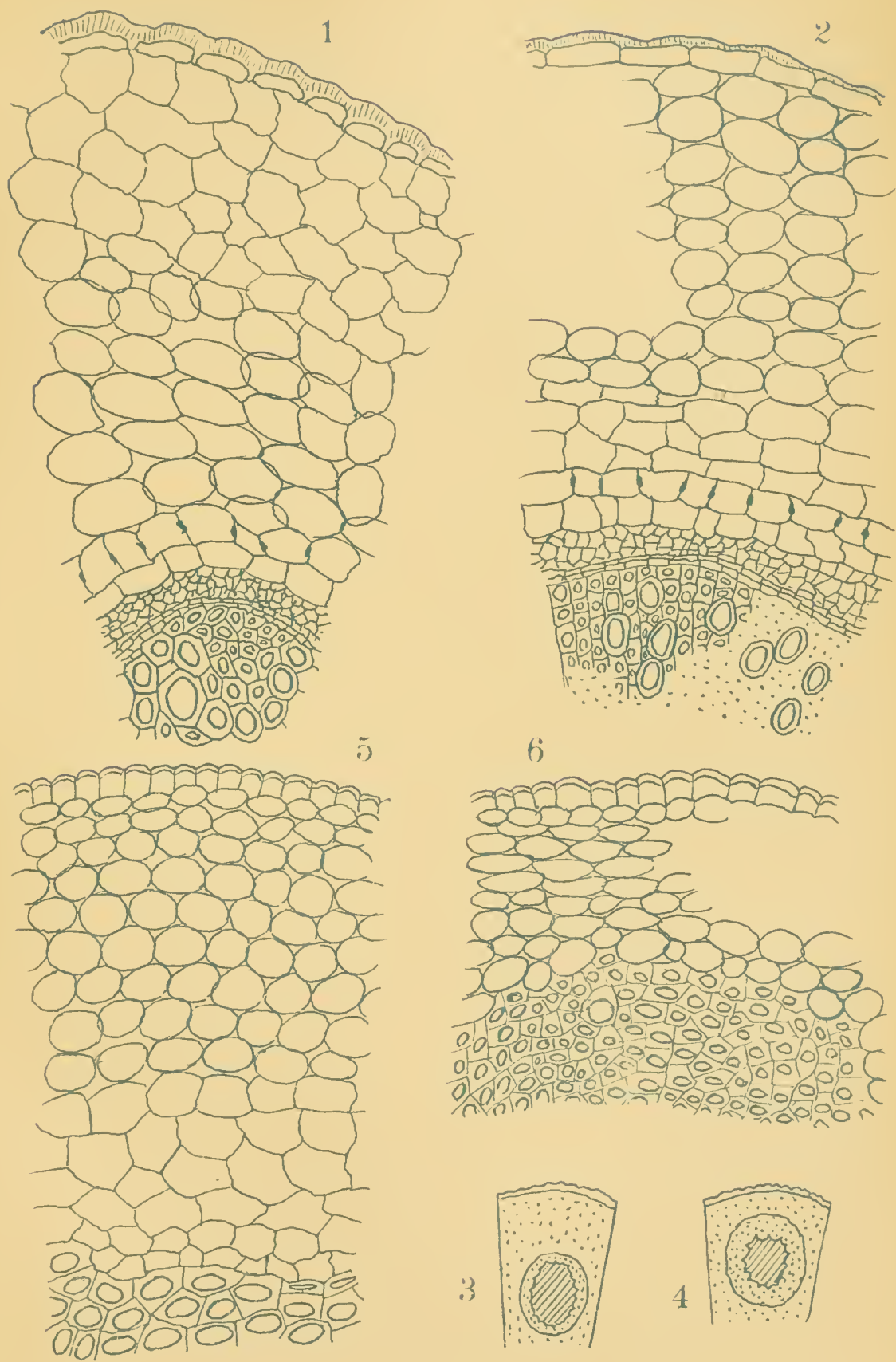

6
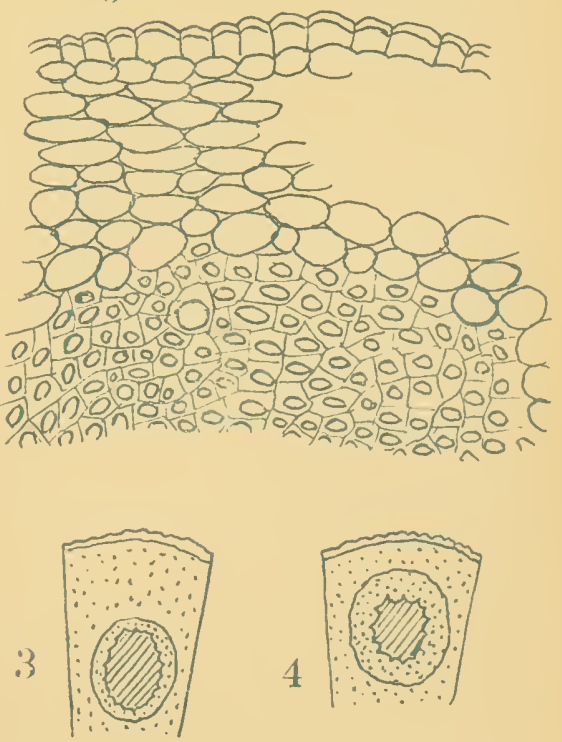


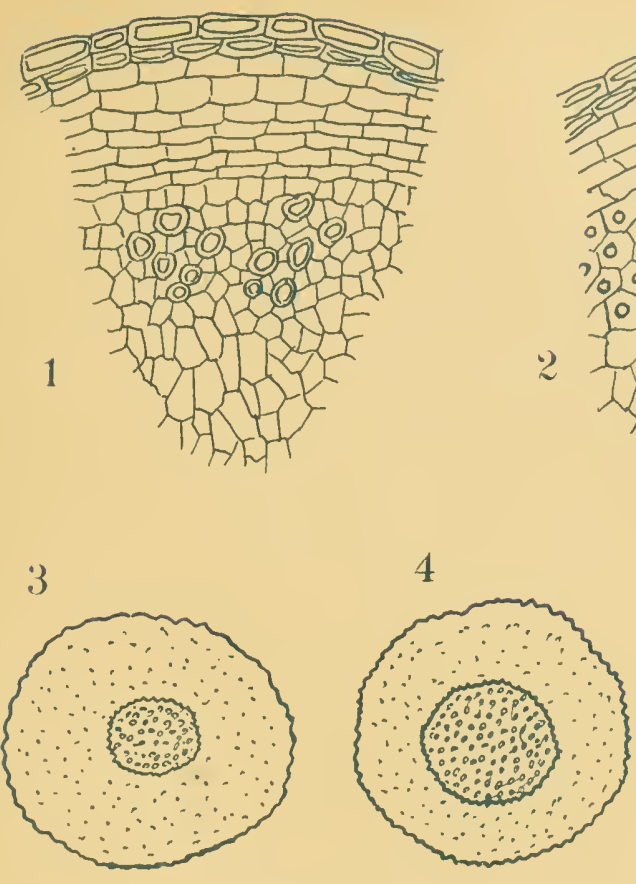

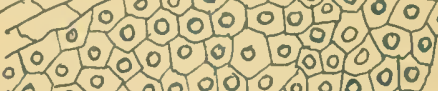

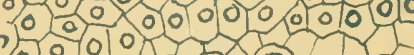

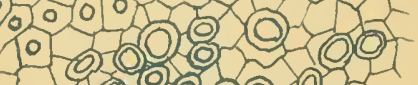

2

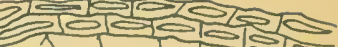

2
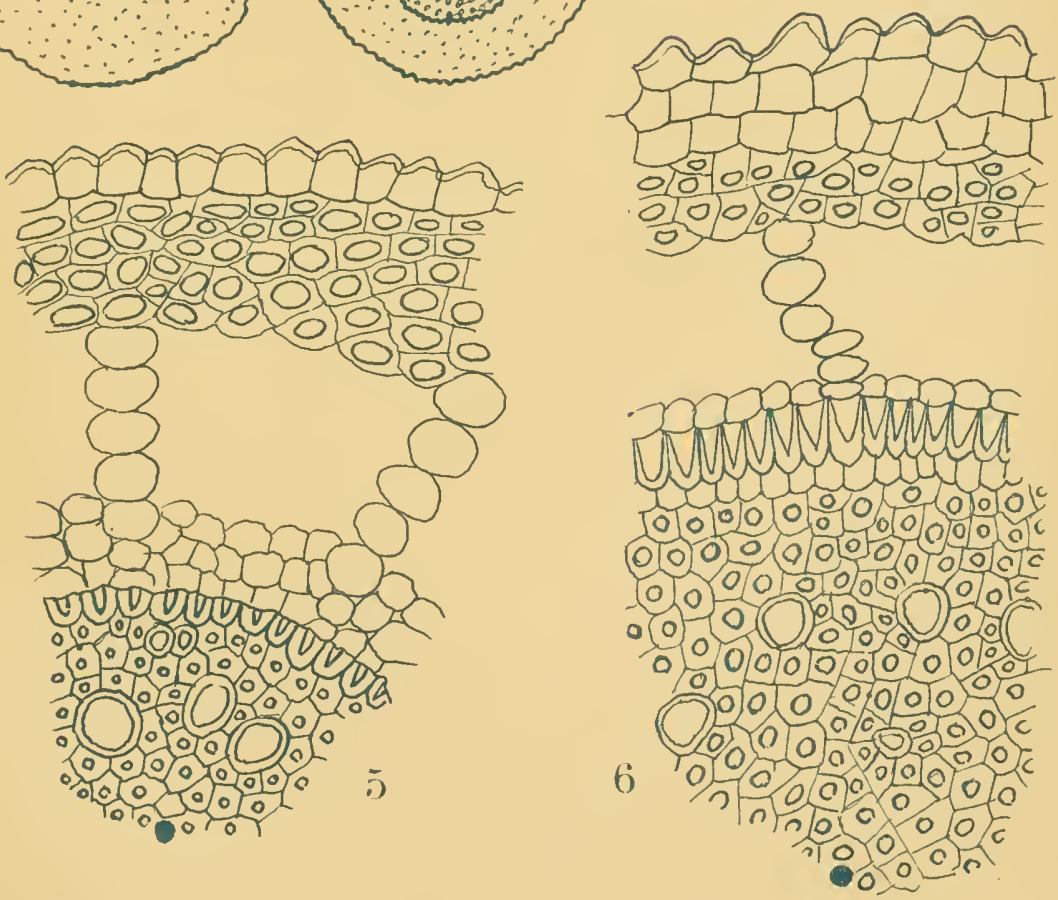

STRUCTURE COMPAREE 



\section{TABLE DES MATIËRES}

PREMIÉRE PARTIE

\section{LE MASSIF SCANDINAVE}

I. GÉLOGIE, OROGRAPIIE, IIYDROGRAPIIE, CLIMATOLOGIE

10 Esousse Grologique

20 Orographe

3o IIYDrographe

zo Climatologie

II. LES ZONES DE VÉGÉTATION

10 Zone de llètre et du Charme

20 Zove Hu CuÈNe

30 Zone de l'Aune blanchithe et des Conifìres

$4^{\circ}$ ZoNe de Bouleau

5 ZONE ALPINE

6o Zone Arctique

FLORULE MARITIME

DEUXIĖME PARTIE

\section{LE MASSIF CENTRAL}

I. GÉOLOGIE, OROGRAPHIE, HYDROGRAPIIE, CLIMATOLOGIE

1 o Géologie

20 Orographie

3o HYDRograpille

$4^{\circ}$ Chimatologie 
II. LES ZONES DE VÉGÉTATION

10 FLORE FOSSILE

20 FLORE ACTUELLE

10 Zone Inférieure ou Région des Vignes

20 Zone Srlvatique Infériedre ou du Cilataignier

80 Zone Srquatique Moyenee ou du Ciline

qo Zone Sylvatigle Supérieute ou Subahipe

$5^{\circ}$ \%ONE ALPINE

6" Flotre Lacistre

$7^{\circ}$ Flone des Tocrbieres

So Florule Halophile

$9^{\circ}$ Flondle Boréale

BIBLIOGRAPHIE

TROISIĖME PARTIE

DIFFÉRENCE ET ANALOGIE DES DEUX FLORES

10 JjFférences Morphologiques

20 Différences Histologįues

$2^{\circ}$ Causes 


\section{ERRATA}

Page 9, Ler alinéa, 5" ligne, lire Torne an lieu de Tornea

) 9, 2 „ $\quad$ S' ligne, live Skagerrak an lieu de Skagerak

"10, 4e \# 3 ligne, lire jerngneiss an lieu de jeergneiss

" 10, 9" " 4. ligne, lire Vestrogothie au liende Vestorgothie

" $13,5^{\prime 3} \quad$ " $\quad$ eligue, live Engelholm au lier de Engelhohn

") $15,20 \quad$ " $13^{\circ}$ ligne, lire Bleking an lieu rle Blekinge

) 18,2 ग 25 ligne, live l'Uppland au lieu de l'Upland

) 20 , 26e ligne, live Fjäll an lieu de Fjull

21, 2e ") $3^{e}$ ligne, live Golfström au lieu de Golfsträm

25, 年 1$)$ Se ligne, live Hedemark au lien de Hedenarken

25, , ") 15 ligne, live Dovrefjeld au lieu de Dovrefjelds

$2-5,5^{e} \quad$ Ireligne, lire il a un au lien de elle a un

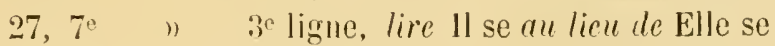

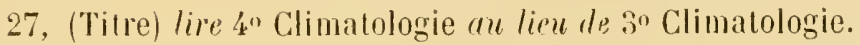

$: 3$, en litre, lire Les Zones de Végétalion an lieu de to Les Zones le Végćtation

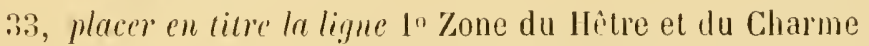

35, à la 9 ligne iles espices herbacés, lire Aingermaland an lieu de Angermanland

:36, Le ligne, live Uppland au lien de Epland

38, dernière ligne live Uppland an licu de Upland

39, derniere ligne, lire Uppland au liru de Upland

4, 4. alinéa, tre ligne, lire altitudinale an lien de altudinale

45, fre alinéa, 3’ ligne, lire particulierement an lien de particuliiment

60, live, $5^{\circ}$ Zone Apine au licu de $5^{\circ}$ La Zone Alpine

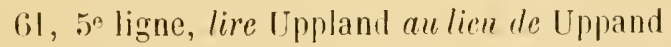

61, même ligne, live glanea an lieu de glanca

63, 20" ligne, lire et de Lule au lieu de et Lule

7:), dernier alinća, lre ligne, lipr abstraction au lien de abstration

:6, dernier alinéa, :s ligne, live silvatique an lien de sylvatipue

100, gr ligne, lire nordselıwedischen an lieu de Nordsehwedischen

101, tre ligne, lire vegretalium an lieu. de vegetatihum

101, 28 ligne, lire Skandinaviens an lieu de Scandinaviens

103, 36 ligue, lire Suecir au licu de Suecirexs 





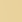





Carlson, C S/Contribution a l'etud || || | | || || || || || | | | | | | | || || || || || || || ||

35185000080299 
